\title{
A Survey on Hybrid Beamforming Techniques in 5G: Architecture and System Model Perspectives
}

\author{
Irfan Ahmed, Senior Member, IEEE, Hedi Khammari, Adnan Shahid, Senior Member, IEEE, Ahmed Musa, \\ Kwang Soon Kim, Senior Member, IEEE, Eli De Poorter, Ingrid Moerman
}

\begin{abstract}
The increasing wireless data traffic demands have driven the need to explore suitable spectrum regions for meeting the projected requirements. In the light of this, millimeter wave (mmWave) communication has received considerable attention from the research community. Typically, in fifth generation (5G) wireless networks, mmWave massive multiple-input multipleoutput (MIMO) communications is realized by the hybrid transceivers which combine high dimensional analog phase shifters and power amplifiers with lower-dimensional digital signal processing units. This hybrid beamforming design reduces the cost and power consumption which is aligned with an energyefficient design vision of 5G. In this paper, we track the progress in hybrid beamforming for massive MIMO communications in the context of system models of the hybrid transceivers' structures, the digital and analog beamforming matrices with the possible antenna configuration scenarios and the hybrid beamforming in heterogeneous wireless networks (HetNET). We extend the scope of the discussion by including resource management issues in hybrid beamforming. We explore the suitability of hybrid beamforming methods, both, existing and proposed till first quarter of 2017, and identify the exciting future challenges in this domain.
\end{abstract}

Index Terms-hybrid beamforming, mmWave, massive MIMO, HetNet, radio access network.

\section{INTRODUCTION}

$\mathbf{R}$ ESEARCH on fifth-generation $(5 \mathrm{G})$ wireless networks, which aims to meet and solve various technical requirements and challenges, has recently received profound attention from academia and industry. A report by Wireless World Research [1] reveals that mobile data traffic is (at least) doubled

The research leading to these results has received the funding from the Agency for Innovation by Science and Technology, Flanders (IWT-V) (under Grant agreement No. IWT140048 SAMURAI Project [www.samuraiproject.be]) and from the European Horizon 2020 Programme (under Grant agreement No. 688116 eWINE Project [www.ewine-project.eu] and 732174 ORCA Project [https://www.orca-project.eu/]), and King Abdulaziz City for Science and Technology (KACST) project PC-37-66.

I. Ahmed (corresponding author) is with the Department of Electrical Engineering, Higher Colleges of Technology, Ruwais, UAE. (e-mail: iahmed3@hct.ac.ae)

H. Khammari is with the Department of Computer Engineering, Taif University, Taif, Saudi Arabia. (e-mail: hkhamari@tu.edu.sa)

A. Shahid, E. De Poorter and I. Moerman are with the IDLab, Department of Information Technology, Ghent University - imec, iGent Tower, Technologiepark-Zwijnaarde 15, B-9052 Ghent, Belgium. (email: Adnan.Shahid@Ugent.be,_Eli.DePoorter@UGent.be and Ingrid.Moerman@UGent.be)

A. Musa is with the department of Telecom Engg., Hijjawi Faculty for Engineering Technology, Yarmouk University, Irbid 21163, Jordan. (e-mail: as.shorman@yu.edu.jo)

K.S. Kim is with the Department of Electronics and Electrical Engineering, Yonsei University, 50 Yonsei-ro, Seodaemun-gu, Seoul 120-749, Republic of Korea. (email: ks.kim@yonsei.ac.kr)

Manuscript received April xx, xxxx; revised August xx, xxxx. every year and will exceed traffic from wired devices by 2018 . In addition, the traffic demand of users has also increased dramatically over the years and this is due to the advent of a broad range of bandwidth-hungry applications such as threedimensional video games, car-to-x (Car2X) communications [2], [3], high resolution augmented reality video streams (not only for gaming but also in factories-of-future) [4]. It is also predicted that by 2020 , there will be around 50 billion devices serving the community [5] and there will be more than six devices per person, which include not only human communications but also machine communications. The aim is to provide an environment in which sensors, appliances, cars, and drones will communicate each other via the cellular network. In order to accommodate such communications, the cellular network has to dramatically increase its capacity. In this regard, in order to accommodate such massive communications, it is forecasted that $5 \mathrm{G}$ network has to provide 1000 times higher capacity than the current system [6]. This ambitious goal will become an inevitable energy crunch problem and thus it is very important to provide energy efficient solutions while maintaining the technical requirements. More than $70 \%$ of the mobile operator electricity bills come from the radio part. In addition, the increased extension of mobile networks contributes significantly to the carbon dioxide emission, which is a major concern nowadays [7]. In order to meet these stringent $5 \mathrm{G}$ requirements and simultaneously maintain the energy efficient design, hybrid beamforming for millimeter wave (mmWave) is envisioned as an integral part of the $5 \mathrm{G}$ wireless networks. A complete list of acronyms is given in Table I.

\section{A. Hybrid beamforming}

The ever increasing demand of wireless communication system depends heavily on spectral efficiency (SE) and bandwidth. Presently, all wireless technologies are operating in 300 $\mathrm{MHz}$ to $3 \mathrm{GHz}$ band [8]. Since the physical layer technology has already touched Shanon capacity [9], the only unexplored option is the system bandwidth. Therefore, the key essence of $5 \mathrm{G}$ wireless networks lies in exploration of high-frequency mmWave band ranging from $3 \mathrm{GHz}$ to $300 \mathrm{GHz}$. On the other hand, multiple-input-multiple-output (MIMO) technology, the use of multiple antennas at transmitter (TX) and receiver (RX), is considered as one of the promising way to improve SE [10]. SE can be improved from MIMO by two ways: 1) a base-station (BS) can communicate with multiple user equipments (UEs) on the same time-frequency-space resources 


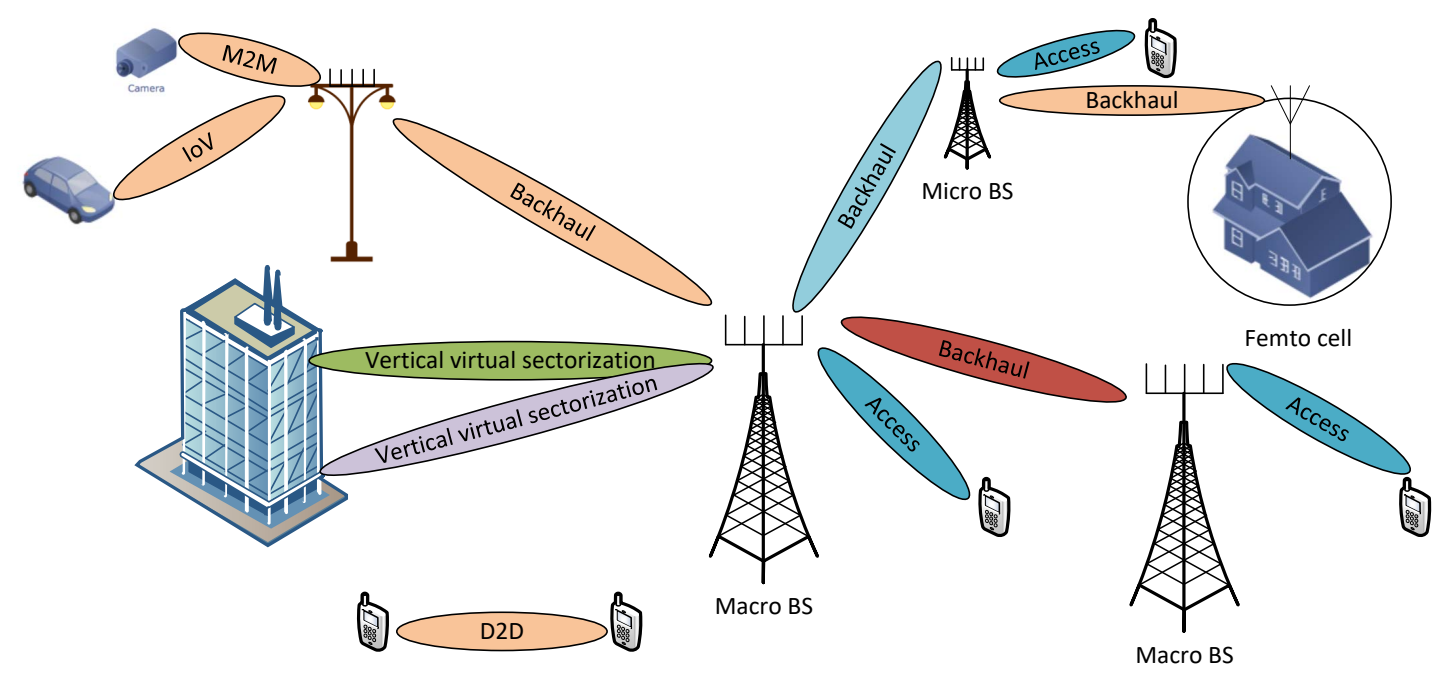

Fig. 1: MmWave massive MIMO beamforming applications in 5G wireless networks.

and 2) multiple data streams are possible between the BS and each UE. The MIMO concept evolves from multi-user (MU)-MIMO to massive MIMO, where the number of antenna elements at BS can reach to hundreds or thousands. Massive MIMO can help in the reduction of small-scale fading and required transmission energy due to beamforming gain [11]. In addition, massive MIMO is essential for mmWave frequencies because it exploits beamforming gain for obtaining sufficient signal-to-noise-ratio (SNR) by combating high pathlosses. Thus, mmWave along with massive multiple-input-multipleoutput (MIMO) communications enable an additional access to the $30-300 \mathrm{GHz}$ bands and improves SE [12], [13].

MmWave massive MIMO will be utilized in a broad range of technologies including machine to machine (M2M), Internet of vehicles (IoV), device to device (D2D), backhaul, access, femto cell (small cell in general), vertical virtual sectorization, etc, where these technologies will be an integral part of 5G wireless networks as shown in Fig. 1. These technologies create significant pressure on cellular network for meeting their communication demands and in this regard, massive MIMO provides capability for enhancing capacity, SE and energy efficiency (EE). But on the other side, the intended frequencies suffer from higher path losses [12]. The use of a multi-tier system can alleviate the path losses problem by providing possible line of sight or fewer multi-paths to the destination [14]. In mmWave massive MIMO systems, the beamforming uses a large antenna array to compensate path losses with directional transmissions. In massive MIMO systems, the traditional baseband digital beamforming (DB) requires one distinct radio frequency $(\mathrm{RF})$ chain per antenna [15], [16], [17]. However, the high power consumption and the high cost of mixed-signal and RF chains (analog-to-digital converters (ADC)/digital-to-analog converters (DAC), data converters, mixers etc.) [18] led to opt for hybrid beamforming operating in the baseband and analog domains. Thus several studies have proposed different architectures aiming to reduce the number of RF chains by combining an analog RF beamformer and a baseband digital beamformer, such techniques are known as hybrid forming methods. Hybrid beamforming methods are designed to jointly optimize the analog and digital beamformers to maximize the achievable rate. Unlike the conventional microwave architecture, the hybrid beamforming architecture seems to be more applicable to mmWave, since the beamforming is performed in the analog domain at RF, and multiple sets of beamformers can be connected to a small number of ADCs or DACs [19].

Beamforming is a procedure, which steers, the majority of signals generated from an array of transmit antennas to an intended angular direction [20]. Specifically, beamforming sends the same symbol over each transmit antenna with a weighted scale factor. At the RX, all received signals are coherently combined using a different scale factor to maximize the received SNR. This gain in SNR in antenna array systems is called beamforming gain. The change in the slope of the error probability resulting from the beamforming gain is called the diversity gain [21]. Interests in hybrid beamforming are motivated by the fact that the number of RF chains is only lower-bounded by the number of transmitted data streams; while the beamforming gain and diversity order are given by the number of antenna elements [22]. In pure digital beamforming, the processing for beamforming is done using a digital signal processor, which provides greater flexibility with more degrees of freedom to implement efficient beamforming algorithms. The pure digital beamforming method requires a separate RF chain for each antenna element, which results in a complex architecture and high power consumption. In the analog beamforming, the antenna weights can either be applied using time delay elements or equivalently phase shifting the signal before RF up-conversion or after the upconversion stage [23]. A detailed comparison of digital and analog beamforming is given in Table II.

Based on the advantages and disadvantages of analog beam- 
forming and digital beamforming, there is a growing interest that hybrid beamforming is a suitable architecture which can exploit large mmWave antenna array with reduced architecture [24], [25]. The hybrid beamforming architectures are broadly divided into two types: fully connected, in which each RF chain is connected to all antennas; and sub-connected or partially connected, in which each RF chain is connected to a set of antenna elements. There exists a complexity-gain trade-off between the two architectures. For fully connected architecture with $N_{t}$ transmit antennas and $N_{R F}$ RF chains, the number of signal processing path is $N_{t} \times N_{R F}^{2}$, whereas, in sub-connected architecture it is $N_{t} \times N_{R F}$. However, the beamforming gain of the fully connected architecture is $N_{R F}$ times greater than the sub-connected architecture [26], [23]. The objective of all hybrid beamforming architectures is to reduce the hardware and signal processing complexity while providing the near optimal performance, i.e., the performance close to the pure digital beamforming.

\section{B. MmWave Massive MIMO Usecases, Products, and Stan- dards}

In this section, we present use cases, products and standards that show the progress of hybrid beamforming.

Usecases: A variety of hybrid beamforming use cases have been demonstrated that shows its suitability in various challenging use cases such a autonomous driving cars, M2M, etc. $5 \mathrm{G}$ wireless systems are targeted in the following areas: Urban Micro (UMi) and Suburban Micro (SMi) [27]. In the UMi environment, the cell radii are kept less than $100 \mathrm{~m}$ and BSs are mounted below the rooftops. Whereas, in SMi environment, the cell radii is around $200 \mathrm{~m}$ and BSs are mounted at $6-8 \mathrm{~m}$. Hybrid beamforming facilitates small packaging of mmWave massive MIMO transceiver that can be deployed at various places; like lamp posts, power lines towers, building corners etc. This was not possible before with digital precoding and beamforming architectures used in MIMO systems due to the cost and power consumption of large number of ADCs/DACs. The small sized BS has envisioned the concept of cluster network where a number of coordinated BSs provide ubiquitous coverage through $\mathrm{BS}$ diversity to compensate the high path losses, diffraction, low penetration and blockages at mmWave frequencies. The quasi line of sight will be ensured through a cluster of BSs, such that in the event of blockage due to the shadowing, one BS will rapidly handoff the UE to another BS in the cluster. In case of mobility these handoffs may be quite frequent. During the transition from fourth generation $(4 \mathrm{G})$ to $5 \mathrm{G}$ a dual connectivity could be provided between Long Term Evaluation (LTE)-Pro and 5G new radio (NR) so that the user can be simultaneously connected to both systems and the radio link connectivity is maintained even if the mmWave system is blocked. At Mobile World Congress (MWC) 2017, Barcelona, Spain; Ericsson showed some interesting use cases of 5G networks [28]. They showed off remote car driving with video and haptic feedback to the driver's seat inside MWC. They used antenna beamforming and antenna beam tracking and a $15 \mathrm{GHz}$ radio to follow the moving cars on a $5 \mathrm{G}$ covered test track. In another demo,
Ericsson Research used a $5 \mathrm{G}$ radio operating at $15 \mathrm{GHz}$ to show how $5 \mathrm{G}$ can be used for combining diverse technologies like radio and fixed access, distributed cloud, machine learning and orchestration into one integrated platform. They separated the control and motor parts of the robot. The control part was moved to cloud where advanced tasks like pattern recognition and machine learning were used in the decision making of the robots' movements and letting it solve the robots' collaborative tasks. The latency in the use-case was upper bounded by 3.5 milliseconds from the robot over the $5 \mathrm{G}$ radio to the cloud execution environment and back to the robot. It has been forecasted that M2M is going to grow at 34\% from 2016 to 2021. M2M IPV6-capable devices will reach 1.8 billion by 2021 [29]. In the next generation massive M2M, devices with the large number of antennas could communicate with each other in a limited area. Precise beamforming can eliminate the inter-device interference and each device can use the same time-frequency resource to communicate with other device [30].

Products: MmWave massive MIMO indoor products have been successfully developed and commercialized. WirelessHD (3.8Gbps) and IEEE802.11ad/WiGig (6.76Gbps) use $60 \mathrm{GHz}$ unlicensed band and target the HD video streaming and Gbps WLAN, respectively [31]. WirelessHD compliant products are Dell Alienware Gaming Laptop M17x, Epson Powerlite Home Cinema Projector, SONY Personal 3D Viewer, ZyXel AeroBeam WirelessHD A/V Kit, Sharp Wireless TX/RX Unit [32]. On the other hand the 802.11ad compliant products include Dell Latitude E7450/70, Intel Tri-Band Wireless, Qualcomm Technologies 802.11ad WiFi client and router solution [33]. Qualcomm and Intel are the major suppliers of the triband chipsets that work in $2.4,5$, and $60 \mathrm{GHz}$ bands.

Standards: 3GPP started standardization of massive MIMO systems with the name of full-dimensional MIMO (FDMIMO, 16 antenna ports) in Release 13 for seamless integration with the current 4G LTE system [34]. It has 2D codebook support for 8-, 12- and 16-antenna ports with reference signal enhancements for beamforming. Though 3GPP standard does not prescribe any particular beamforming architectures, the design of channel state information (CSI) acquisition protocols in Release 13 of LTE-Advanced Pro is closely related to the sub-connected hybrid beamforming structure. The FD-MIMO uses sub-connected-like structure for the transmission of the beamformed pilots [22]. Field trials of the FD-MIMO proofof-concept systems have demonstrated its potential gain in terms of the coverage and capacity. Further enhancement in multiuser massive MIMO system has been proposed in Rel-14 [35] which supports up to 32-antenna with advanced codebook design for high resolution beamforming [36]. These developments are steps towards 3D hybrid beamforming in mmWave massive MIMO framework of 5G networks. Qualcomm has announced the first Cellular Vehicle-to-Everything (C-V2X) trial with Audi and others based on 3GPP Rel-14 [37]. The 5G New Radio (NR) has been recently added as a working item in 3GPP Rel-15 [38], which is expected to be deployed in 2019+. Qualcomm has started working on Rel-15 5G NR enhanced mobile broadband (EMBB) design, demos, and trials. The 5G $\mathrm{NR}$ will integrate multiuser massive MIMO, mmWave, hybrid 
TABLE I: Summary of important acronyms

\begin{tabular}{ll}
\hline Acronyms & Meaning \\
\hline 4G & Fourth generation \\
5G & Fifth generation \\
AB & Analog beamforming \\
ADC & Analog-to-digital converter \\
AoA & Angle of arrival \\
APAVSM & Analog precoding aided virtual space modulation \\
APM & Amplitude-and-phase modulation \\
AWGN & Additive white Gaussian noise \\
BER & Bit error rate \\
BS & Base-station \\
CAP & Continuous aperture phase \\
Car2X & Car-to-x communication \\
cmWave & Centimetre wave \\
CNAS & Critical number of antennas searching \\
CRAN & Cloud radio access network \\
CSI & Channel state information \\
D2D & Device to device \\
DAC & Digital-analog converter \\
DB & Digital beamforming \\
DC & Digital combining \\
DP & Dual polarized \\
EE & Energy efficiency \\
EEHP & Energy efficient hybrid precoding \\
EGT & Equal gain transmission \\
FDD & Frequency division duplex \\
GP & Gradient pursuit \\
HetNet & Heterogeneous wireless network \\
IoT & Internet of things \\
IoV & Internet of vehicles \\
LTE & Long term evaluation \\
M2M & Machine to machine \\
MAC & Medium access control \\
MIMO & Massive multiple-input multiple-output \\
MLS & Magnitude least square \\
mmWave & Millimeter Wave \\
MRT & Maximal ratio transmission \\
MSE & Mean square error \\
MU-MIMO & Multi-user multiple-input multiple-output \\
MU-MISO & Multi-user multiple-input multiple-output \\
\hline &
\end{tabular}

\begin{tabular}{ll}
\hline Acronyms & Meaning \\
\hline OFDM & Orthogonal frequency-division multiplexing \\
OMP & Orthogonal matching pursuit \\
OSA & Overlapped sub array \\
PAA & Planar antenna array \\
PS & Phase shifter \\
QoE & Quality of experience \\
QoS & Quality of service \\
RAN & Radio access network \\
RAT & Radio access technology \\
RF & Radio frequency \\
RX & Receiver \\
SBS & Small cell base station \\
SCM & Spatial covariance matrix \\
SE & Spectral efficiency \\
SINR & Signal-to-interference-plus-noise ratio \\
SM-MIMO & Spatial modulation multiple-input multiple-output \\
SNR & Signal-to-noise ratio \\
SON & Self-organized networks \\
SU-BF & Single-user beamforming \\
SU-SM & Single-user spatial multiplexing \\
SVD & Singular value decomposition \\
TDD & Time division duplex \\
TDMA & Time-division multiple access \\
TX & Transmitter \\
UE & User equipment \\
UENO & User equipment number optimization \\
ULA & Uniform linear array \\
UPA & Uniform planar array \\
ZF & Zero forcing \\
$F A B$ & Analog precoder \\
$F D B$ & Digital precoder \\
$N_{R F}$ & Number of RF chains \\
$N_{S}$ & Number of data streams \\
$N_{c l}$ & Number of clusters \\
$N_{r a y}$ & Number of multipath rays \\
$W^{A B}$ & Analog combiner \\
$W^{D B}$ & Digital combiner \\
& \\
\hline & \\
\hline & \\
\hline & \\
\hline & \\
\hline
\end{tabular}

TABLE II: Comparison of digital and analog beamforming

\begin{tabular}{llllllll}
\hline Beamforming & Degree of freedom & Implementation & Complexity & Power consumption & Cost & Inter-user interference & Data streams \\
\hline Digital & High & ADC/DAC, mixer & High & High & High & Low & Multiple \\
Analog & Low & Phase shifters & Low & Low & Low & High & Single \\
\hline
\end{tabular}

beamforming, scalable OFDM numerology, advanced LDPC channel coding, time division duplex (TDD) subframe, and low latency slot structure [37].

\section{Requirement and importance of hybrid beamforming struc- tures}

It is envisioned that hybrid beamforming architectures will leverage the benefits of analog as well as digital beamforming. In this perspective, a hybrid beamforming architecture with analog phase shifters has emerged as an attractive proposition for the next generation mmWave massive MIMO systems [39], and HetNets [40]. The hybrid beamforming scheme has particular importance in the context of massive MIMO in view of its reduced hardware cost and power consumption [41].
In mmWave massive MIMO, the high cost and power consumption of mixed analog/digital signal components per antenna makes it infeasible to perform all signal processing tasks at baseband [12], [42]. This motivates the design of different architectures of the hybrid beamforming and the analysis of their impact on the signal processing, precoding, combining and channel estimation [43], [44]. The hybrid beamforming architecture consists of a reduced number of RF chains which facilitates multi-stream digital baseband processing followed by analog (baseband or RF) processing to realize antenna beamforming gain [23].

MmWave, massive MIMO, and hybrid beamforming can potentially solve many technical issues for $5 \mathrm{G}$ wireless network ranging from the capacity enhancement to the energy 
consumption reduction and they can be seamlessly integrated with heterogeneous network (HetNet) for meeting the overall requirement of the $5 \mathrm{G}$ wireless networks. HetNet will play a key role in in $5 \mathrm{G}$ wireless networks. It comprises a high power macro BS (MBS) and is underlaid with low powers BSs including pico cell, femto cell, which are generally referred as small cells. HetNet is typically used for enhancing the capacity, coverage area and EE by reusing the spectrum. On the other hand, network densification through the massive deployment of cells of different types (macro, micro, pico, and femto cells) is a key technique to increase the network capacity and EE. It is expected that relaying and multihop communications be among the central elements of the 5G wireless network architecture [45]. Particularly, massive MIMO communication enabled by mmWave frequency and hybrid beamforming can be used for both outdoor (pointto-point backhaul links) and indoor (supporting multimedia enriched application). MmWave technology has already been utilized in IEEE 802.11ad and IEEE 802.15.3.c. However, it has not been adequately explored for the cellular environment because of many reasons including high propagation losses, rain fading, absorption and scattering of gases [46]. In HetNet both mmWave and microwave frequencies could be used in parallel depending upon several factors such as the regulatory issues, application, channel, and path loss characteristics of various frequency bands [47]. The feasibility of hybrid beamforming in small cells has been evaluated in [48] with the help of a real-time software-defined radio prototype testbed. In a small cell setup where the number of users is generally small, the massive MIMO capacity gains can be achieved with simple and uncoordinated maximal ratio transmission (MRT) or zero-forcing (ZF) hybrid beamforming schemes (when there is no pilot contamination) [47]. Also, massive MIMO communications enabled by mmWave frequencies and hybrid beamforming can be used for point-to-point backhaul links or for supporting asymptotic orthogonality in point-tomultipoint access links.

The basic advantages offered by the features of hybrid beamforming signal processing can be summarized as follows:

- An enabler of mmWave massive MIMO communications: As mmWave massive MIMO is an enabler of $5 \mathrm{G}$ wireless networks with $1000 \times$ capacity enhancement, the hybrid beamforming is an enabler of mmWave massive MIMO communications. Without the hybrid beamforming, the mmWave massive MIMO is either costly and complex (digital beamforming) or prone to beamforming inaccuracy and inter-users interference (analog beamforming) [23].

- Reduced capital hardware cost: Hybrid beamforming reduces the capital cost by reducing the required number of RF chains at TX and RX sides compared to the digital beamforming system with the same number of antennas [49].

- Energy efficiency: Compared to the sub $6 \mathrm{GHz}$ MIMO systems, in mmWave massive MIMO, the large antenna arrays are realized by hybrid beamforming which potentially reduces the downlink and uplink transmit power through coherent combining and an increased antenna aperture [50]. It can increase the EE in uplink by reducing the required transmit power of each user equipment (UE) which is inversely proportional to the number of antennas at the BS with no reduction in performance [50], [51].

- Reduced operational cost: Due to the feasibility of increased number of antennas, the large array of antennas allows the use of low cost RF amplifiers in the milliWatt range, because the total transmitted power $\propto 1 / N_{t}$ [52], [17]. This in contrast to the classical antenna arrays, which use few antennas fed from a high-power amplifier at low power efficiency.

\section{Challenges in hybrid beamforming and motivation}

It is very difficult to find the analog and digital beamforming matrices that optimize, e.g., SE in downlink or uplink constraint to the constant amplitude phase shifters and the number of RF chains [42]. The main difficulties include: i) analog and digital precoders at the transmit end, as well as combiners at the receive end, are coupled, which makes the objective function of the resulting optimization non-convex, ii) typically the analog precoder/combiner is realized as a phase-shifter network, which imposes constant modulus constraints on the elements of the analog precoding and combining matrices [53], iii) when using the practical finite-resolution phase shifters, the optimal analog beamformer lies in a discrete finite set, which typically leads to NP-hard integer programming problems [54], iv) the difficulty in CSI availability at BS TX side, because the mapping between transceivers and antennas (except the oneto-one mapping) makes channel estimation in both downlink and uplink more complicated [55].

Given the importance of hybrid beamforming in mmWave massive MIMO communications, the proposed solutions for implementation of the hybrid beamforming, and the existing issues and challenges, we provide in this paper an extensive survey of state-of-the-art proposals, approaches, and the open research issues in hybrid beamforming architectures, analog and digital signal processing and HetNet as an application of mmWave massive MIMO communications.

\section{E. Scope and Organization}

In this paper, we present a comprehensive survey of hybrid beamforming at architecture, signal processing and HetNet (at an application level). The scope of the paper covers important aspects of hybrid beamforming including hardware architecture (including fully connected and partially connected architectures), analog and digital signal processing for various UE/BS configurations, resource management, and HetNet which is an intrinsic application of mmWave massive MIMO communications. This survey paper is intended for the researchers in the field of hybrid beamforming. We do not review the basics of mmWave frequencies, channel models, and massive MIMO, as there exist ample review literature in these fields. For example, for review in mmWave communication we refer to [56], for massive MIMO we refer to [16], [57], for general beamforming in indoor and outdoor mmWave communications we refer to [23], for hybrid beamforming 
categorization on the basis of instantaneous and average CSI we refer to [22]. However, there are other important aspects of hybrid beamforming, like EE, hardware impairments, etc, that we are thoroughly presenting in this paper.

This paper is organized as follows: In Section II we present the review of survey papers on mmWave, massive MIMO, and beamforming technologies. Section III is about the different hybrid beamforming architectures. Section IV lists the various system models of hybrid beamforming along with the solution methods for obtaining digital and analog beamformers. Section $\mathrm{V}$ discusses resource management issues for hybrid beamforming architectures. Hybrid beamforming in small cells (HetNets) is covered in Section VI, followed by the conclusions in Section VII.

\section{Review of Survey Papers on MmWave, Massive MIMO, AND BEAMFORMING}

In this section, we review recent survey papers on mmWave, massive MIMO and beamforming in 5G wireless networks.

The future $5 \mathrm{G}$ wireless network has to bear various challenges in terms of increased capacity, traffic demand, etc. which can be solved by variety of advanced features, such as network densification, massive MIMO antenna, coordinated multi-point processing, inter-cell interference mitigation techniques, carrier aggregation, and new spectrum exploration. A compressive analysis of proposed developments and technologies to enable 5G technology is given in [58]. This paper describes research studies about Orthogonal Division Multiple Access (OFDMA), mmWave communication, energy efficient D2D communication, etc. The paper highlights key features of 5G mobile technology, i.e., flexibility, accessibility, and cloudbased service offerings. These key features are going to enable the futuristic mobile communication technology. It is shown in [59] that mmWave beamforming is one of enabling technology of $5 \mathrm{G}$.

Reference [60] describes 5G wireless network architectures and their enabling technologies. These technologies aim to enable the 5G network architecture to fulfill the users demand such as high capacity, high data rates, and high quality of service. Furthermore, this paper addresses some of these enabling technologies such as spectrum sharing with cognitive radio (CR), mmWave solutions for the $5 \mathrm{G}$ networks, multiradio access technology, full duplex radio, cloud technologies for $5 \mathrm{G}$ radio access networks (RANs), etc. The authors propose a general architecture for $5 \mathrm{G}$ network which will encompasses direct D2D, Internet of Things (IoTs), small cell access points, and network cloud. 5G mobile networks are featured by heterogeneous connectivity, zero latency, and ultrahigh data rates. In [61], the authors tent to answer "what will be done by $5 \mathrm{G}$ and how?". The author discusses the limitations of $4 \mathrm{G}$ cellular networks and new features of $5 \mathrm{G}$ networks. In addition, their paper classifies the proposed architectures based on EE, network hierarchy, and network types. The author highlights various issues such as interference, quality of service (QoS), handoff, security-privacy, channel access, and load balancing that affects the realization of $5 \mathrm{G}$ networks. Finally, the paper discusses the feasibility of current trends in research on $5 \mathrm{G}$ networks through an evaluation on real testbeds and experiments. The authors of [62] present the latest technologies, including innovations in wireless networks, next generation Internet, content networks, and other related area. The authors outline the latest research and new paradigms for wireless technologies, access networks, content delivery networks, components for server side and mobile device side, open source communities, and services.

Since there is a difference in the characteristics of mmWave to the conventional signals, PHY and MAC layer aspects need to be changed to visualize the concerned benefits of the hybrid beamforming in mmWave systems. The authors in [63] make an exhaustive survey of next generation $5 \mathrm{G}$ wireless networks. The paper begins by pointing out the new architectural changes associated with the RAN design, including air interfaces, smart antennas, cloud and heterogeneous RAN. Thereafter, the paper presents an in-depth survey on underlying mmWave physical layer technologies, encompassing new channel model estimation, directional antenna design, beamforming algorithms, and massive MIMO technologies. After that, the details of medium access control (MAC) layer protocols and multiplexing schemes needed to efficiently support this new underlying physical layer are elucidated. Next, the paper sheds the light on new QoS, quality of experience (QoE), and self-organized networking (SON) features associated with the $5 \mathrm{G}$ evolution. In addition, the authors provide a detailed review on energy awareness and cost efficiency to mitigate the network energy consumption and operating expenditure. Furthermore, the authors discuss relevant field trials, drive tests, and simulation experiments.

The author in [64] summarizes the key activities toward 5G wireless network in particular those conducted in Europe. The paper reviews recent thematic IEEE Communications Magazine $5 \mathrm{G}$ issues and relevant white papers from different sources such as Nokia, Huawei, etc. The paper sheds the light on what $5 \mathrm{G}$ is about: what are the building blocks of core $5 \mathrm{G}$ system concept, what are the main challenges and how to tackle them. The paper states that the performance enhancements are mainly expected from integrating the network densification (e.g., small cells, D2D), increased spectrum (enhanced carrier aggregation, spectrum sharing, beyond $6 \mathrm{GHz}$ frequencies), and enhanced wireless communication technologies (e.g., massive MIMO, new waveforms, virtual zero latency radio access technologies (RATs). From the standardization bodies, it is also evident that mmWave, massive MIMO and beamforming will be an integral part of $5 \mathrm{G}$ systems.

From the papers [62]-[64], it is evident that in order to meet such ambitious $5 \mathrm{G}$ requirements, various potential technologies such as D2D, ultra-dense small cells, Internet of things, etc will be an integral part of $5 \mathrm{G}$ network. However, mmWave, massive MIMO, and beamforming will further assist in improving the performance of the technologies and subsequently, help in attaining the ambitious $5 \mathrm{G}$ requirements. In the following sub-sections, we provide review of survey papers on the hybrid beamforming architecture, analog and digital beamforming techniques for mmWave and massive MIMO systems, and beamforming in small cells. 


\section{A. Hybrid Beamforming Architectures}

In contrast to general 5G overview papers, there are only few papers in literature that illustrates the hardware aspects of hybrid beamforming. In [22], the authors categorized hybrid beamforming architectures for the downlink transmission at BS according to the required CSI, the complexity (full complexity, reduced complexity, and switched), and the carrier frequency range (cm-wave versus mmWave). The utilization of the large array antenna elements aims to increase the capacity gains in massive MIMO systems. Based upon the analysis provided in the paper, it is clear that there is no single hybrid beamforming structure that can provide the best trade-off between complexity and performance. Thus, in order to get the best performance from hybrid beamforming, the structure needs to be dynamic based upon the application and channel conditions. Typical mmWave hybrid beamforming architectures, signal processing algorithms, and RF system implementation aspects are described in [23]. In addition, determination of the optimal number of RF chains (which has a direct impact on the complexity, cost and power consumption) is carried out under practical constraints such as the number of multiplexed streams, antenna elements, constant amplitude and quantized phases of the analog phase shifters, is generally based on the sum rate maximization [23]. The authors present hybrid beamforming architectures, signal processing algorithms and implementation aspects for various indoor and outdoor environments such as WPAN, WLAN and outdoor, which can scratch many use cases for visualizing hybrid beamforming in a $5 \mathrm{G}$ network.

Heath et al. [12] classify the hybrid beamforming on the basis of analog beamforming components. The analog beamforming can be implemented either by digitally controlled phase shifters, electronic switches or lens antenna. The digitally controlled phase shifters can eliminate the residual interference between data streams but they suffer from high power consumption and the quantization error because only finite step phase shifts are available. Alternatively, switch based analog combiner exploits the sparse nature of mmWave massive MIMO channel and only a subset of antennas is selected instead of an optimization over all quantized phase values. The third approach uses lens antenna for the analog beamforming at the front-end. In this architecture, the continuous aperture lens antenna steers the beam, controlled by the mmWave beam selector. The lens based front-end can be realized by the unitary discrete Fourier transform matrix. However, this paper only presents the various design of analog beamforming without discussing the detail of digital counterpart. The hardware complexity of hybrid beamforming in terms of ADC resolution is surveyed in [65]. It has been shown that low resolution ADC (1-bit ADC) has gained much attention in research community. Although 1-bit ADCs impose EE but suffer from rate loss and require long training sequence for channel estimation. This paper does not account the other hardware components like phase shifters or switches in different hybrid beamforming architectures.

In summary, none of the aforementioned paper comprehensively reviews the architecture, performance, and the com- plexity of the hybrid beamforming. Whereas, in this paper, we provide the complete review of hybrid beamforming architectures, namely: fully-connected, partially- or subconnected, fully-connected with virtual sectorization, dynamic subarray, and hybrid beamforming with low complexity analog beamforming. Also, we compare various hybrid beamforming architectures on the basis of power hungry ADCs and phase shifters.

\section{B. Analog and Digital Beamforming Techniques for MmWave and Massive MIMO Systems}

Next generation cellular networks will utilize mmWave to support more users and to achieve higher data rates. However, network nodes tuned at mmWave experience small coverage area problems as well as outdoor penetration difficulty. The main challenges in mmWave cellular networks are found in spatial management, link margin operation, interference management, object blockage, etc. The link margin, for instance, can be overcome by enabling beamforming approach in high directional antenna arrays (massive MIMO). Authors in [56] conduct a survey to delve deeper into proposed solutions for combating the mmWave challenges. In the light of these solutions, architecture and protocols for mmWave communications have been proposed. Furthermore, mmWave applications in $5 \mathrm{G}$ wireless networks such as wireless backhaul, small cell access, etc., are presented. Some investigations to unveil open research issues related to mmWave of $5 \mathrm{G}$ have been outlined that will help continuing the development of mmWave for $5 \mathrm{G}$ wireless network.

Massive MIMO possesses many potential benefits including extensive use of inexpensive low-power components, reduced latency, MAC layer simplification, and robustness against intentional jamming. However, massive MIMO technology uncovers new problems such as pilot contamination which needs to be immediately addressed. Accurate and timely CSI estimation is vital for wireless communications. In case of multiuser MIMO or massive MIMO communications, this CSI becomes more important to enable the multiple streams and eliminate the inter-user interference. The estimation of the CSI is carried out by the training sequences (also called pilots). The pilot contamination is caused by the inter-cell and intracell interference during pilot transmissions from the UEs to BS. In this context, the reference [66] provides an up-todate survey on pilot contamination and presents the major sources that impact the massive MIMO system performance using TDD. These sources include non-reciprocal transceivers and hardware impairments, etc. Subsequently, the paper reviews and categorizes some of the established theories that analyze the effect of pilot contamination on the performance of massive MIMO systems. Finally, the paper outlines the open research issues of pilot contamination which include computational complexity, training overhead, and channel reciprocity usage. The paper addresses and proposes a real challenge in massive MIMO. In addition, the investigation will be helpful in the standardization and the ongoing research on mmWave and massive MIMO. But it lacks the review of the hybrid beamforming design techniques in the presence of pilot 
contamination and the channel estimation errors. The largescale MIMO or massive MIMO system has been considered as a promising technology for the beyond 2020 next generation network. In this regard, the authors in [57] provide a survey on the state-of-the-art of massive MIMO systems. The paper classifies and analyzes their typical application scenarios. In addition, the paper sheds the light at channel measurements and channel models alongside with a variety of open research issues. The authors also present key techniques for both the physical and network layers. The authors not only address the physical and networking techniques but also present the key application scenarios, which can facilitate the implementation of hybrid beamforming while considering massive MIMO. Potential 5G physical layer technologies including mmWave and massive MIMO in small cells are discussed in [67]. It provides a brief description of technical challenges and the recent results.

The aforementioned survey papers either discuss the characteristics of mmWave frequencies and channel, and the challenges in the interference management and pilot contamination or the potential applications of massive MIMO in 5G, like backhaul, HetNet, software defined architectures, small cells, adaptive beamforming etc. In this paper, we focus on the digital and analog beamforming techniques in the mmWave massive MIMO systems for various possible number of nodes and antennas.

\section{Small Cells and Beamforming}

It is expected that the $5 \mathrm{G}$ network will greatly depend on small cell base-stations (SBSs) and multiple antennas technologies. The paper [68] reviews state-of-the-art literatures related to the applications and challenges associated with using multiple antennas in SBSs. This paper presents design challenges associated with size, cost, and performance in SBSs. In addition, it also provides insights to the design challenges faced by the possible futuristic networks using new frequency bands. Since small cells will be an integral part of the $5 \mathrm{G}$ network, it is very important to see the issues that are related with mmWave and beamforming that the authors present in the paper.

Another article [47] explores network densification in multiple dimensions, including deployment of superdense HetNets with different types of cells, multiple RATs, massive MIMO at BSs and/or UE, and exploitation of both the microwave/mmWave frequency bands. The authors discuss the key benefits and challenges of $5 \mathrm{G}$ wireless HetNet that integrates massive MIMO and mmWave technologies. This unparalleled network should exploit the developments including superdense and heterogeneous deployment of cells. The paper describes the potential HetNet architecture as well as its design and technical challenges that employs microwave / mmWave frequency bands. Next, the paper presents three case studies addressing some of the challenges and showing their benefits. This paper lays out full-scale 5G HetNet architecture and highlights the challenges that must be combated to pave the way of $5 \mathrm{G}$ network. It is important to realize network densification in multiple dimensions, including deployment of superdense HetNets with different types of cells, multiple RATs, massive MIMO at BSs and/or UE, and exploitation of both the microwave and mmWave frequency bands. HetNet is also considered as a pioneering technology to enable cellular networks to evolve to greener 5G systems [69]. Optimizing energy consumption in HetNets can be achieved by dynamically switching off BSs. This will potentially improve the overall network performance. Several strategies basis for switching off BSs have been proposed according to different design prospective such as random, distance-aware, load-aware, and auction. In addition, many studies mainly in cloud access networks have been done to integrate BS switch-off strategies with other strategies such as user association, resource allocation, and physical-layer interference cancellation. Reference [69] summarizes the state-of-the-art BS switch-off strategies on the basis of optimization objective and the constraints, specific BS-switching techniques, joint BS-switching and another $5 \mathrm{G}$ enabling strategy, and BS-switching techniques in cloud-RAN (C-RAN)/heterogeneous C-RAN in tabular forms. Due to the densification of small cells in future $5 \mathrm{G}$ networks, an energyefficient concern has gained paramount importance. Among various possibilities, the paper suggests an attractive technique for conserving the energy by switching off BSs based upon certain criterion.

The authors in [67] discuss the daunting requirements for $5 \mathrm{G}$ networks. These requirements include high frequency spectra with large bandwidth, BS densification, and huge numbers of antennas to support gigantic data traffic. These requirements have driven many studies, researches, etc. to innovate new technologies. This paper highlights several design choices, key features, and technical challenges that provides deep understanding of $5 \mathrm{G}$ networks. The article states that the physical layer technologies such as spatial multiplexing using multiuser MIMO (MU-MIMO) techniques with mmWave in small cell geometry appears as a promising technology to increase throughput. However, the paper does not provide a final solution and states that these inevitable technologies have many technical challenges which open the door for further research studies. The paper also presents a table that shows the impacts of the most significant techniques as well as the pros and cons of their use in 5G wireless networks. In 5G ultradense small cell networks, the SBS would be connected to the core network/macrocell BS over mmWave massive MIMO link [70]. The high directional beam along with the spatial multiplexing enables the point-to-multipoint communication links at the same time and frequency without interference. The 5G backhaul technology forks many challenges: (1) >10 Gbps capacity, (2) <1 msec end-to-end latency, (3) high security and resilience, (4) time and frequency synchronization, (5) low energy consumption and low cost [70]. The authors in [70] identify six key research directions including mmWave massive MIMO that would jointly pave the way to $5 \mathrm{G}$ backhaul.

From this review of survey papers on $5 \mathrm{G}$ technologies and the summary in the Table III, it can be seen that only few papers briefly cover the hybrid beamforming techniques. In [23], authors analyze the evolution and advancements in antenna beamforming for mmWave communications in the 
context of the distinct requirements for indoor and outdoor communication scenarios till midyear 2015. A CSI based classification of hybrid beamforming structures has been presented in [22]. There is no other survey paper on this topic and non of these two papers touches the different hybrid beamforming scenarios arise from the different antenna configurations systematically. Since hybrid beamforming is born with massive antenna elements, in this paper we review the hybrid beamforming architectures, signal processing solutions related to various massive MIMO configurations in system models, and the application of hybrid beamforming in the HetNets. We discuss all the techniques that have been used in the literature pertaining directly or indirectly to the hybrid beamforming. The taxonomy of hybrid beamforming used in this paper is shown in Fig. 2. The taxonomy starts with a generalized architectures which include fully-connected and sub-connected. Then, the system model perspectives (which include various antenna configuration on BS and UE side) is presented. More specifically, the impact of various antenna configurations in different technologies such as D2D, backhaul, inter-cell interference (pilot contamination), etc are analyzed for hybrid beamforming systems. In addition, resource management and application which can have direct and indirect impact on the performance of hybrid beamforming are surveyed. Although the resource management issues are not directly related to hybrid beamforming, they have an indirect impact on the performance of hybrid beamforming systems such as by beam management, initial search and tracking, etc. Lastly, the issues of hybrid beamforming in HetNets are explored and the associated research challenges are also marked for the further performance improvement of HetNets by utilizing the hybrid beamforming architecture.

\section{HyBRID BEAMFORMING ARCHITECTURES}

The ideal mmWave massive MIMO hardware realization requires number of RF chains equal to the number of antennas. The RF chain consists of hardware components such as ADC/DAC, mixer, etc. On the other hand mmWave wireless channel does not encourage multipath reflections and offers a sparse channel between TX and RX. These two conditions motivates the design of hybrid beamforming with the number of RF chains as low as the required data streams. The increase of energy consumption in massive MIMO antennas technology adopted for $5 \mathrm{G}$ wireless communication systems is mainly due to the large number of antennas and RF chains. Large antenna arrays will be intensively used in the future mmWave cellular networks, and different possible antenna architectures and MIMO techniques will be needed. Instead of implementing a fully digital beamforming, which requires one distinct RF chain for each antenna, a two-stage hybrid linear precoding and combining scheme is a possible solution. In contrast to centimeter wave (cmWave) communication systems which make the use of large antenna arrays at either TX or RX or both to have sufficient link margin, the mmWave communication systems are much more compact and require fewer spatial degrees of freedom for parallel multi-stream transmission [22]. Hybrid analog and digital beamforming is an emerging technique for large-scale MIMO systems since it can reach the performance of the conventional fully digital beamforming schemes, with much lower hardware implementation complexity and power consumption. The green design of the RF chains, the use of simplified TX/RX structures, and the energyefficient design of power amplifiers are among the hardware solutions to improve the EE, especially in systems with many antennas such as massive MIMO systems and mmWave systems. Hybrid analog and digital beamforming structures have been proposed to lower energy consumption for mmWave communications [73]. Digital beamforming offers better performance at increased complexity and cost. In contrast, the analog beamforming is a simple and cost effective method with less flexibility. The Hybrid beamforming architecture provides sharp beams with phase shifters (PSs) at analog domain and flexibility of digital domain [74]. The vulnerability to blocking and the need for strong directionality are the most important physical challenges for mmWave cellular systems. The authors of [41], review mathematical models for mmWave cellular systems and analyze the fundamental physical differences from conventional Sub-6GHz cellular systems. The need for significant directionality at the TX and/or RX, is achieved through the use of large antenna arrays of small individual elements [41]. A fully-digital processing is hard to realize at mmWave frequencies with wide bandwidths and large antenna arrays, because the baseband precoding/combining processing assumes that the transceiver dedicates an RF chain per antenna as shown in Fig. 3(a). An analog beamforming can be implemented using networks of phase shifters as shown in Fig. 3(b), but it consumes high power and cannot provide the data streams multiplexing. The hybrid precoding architecture (as in Fig. 3(c)), provides a flexible compromise between hardware complexity and system performance [41]. The hybrid beamforming structure are proposed as an enabling technology to obtain the benefits of MIMO and also to provide high beamforming gain to overcome the high propagation loss in mmWave bands, for 5G cellular communications [59].

\section{A. Fully-connected and Sub-connected Hybrid Beamforming Structures}

In a fully connected hybrid beamforming structure, each RF chain is connected with all antennas, and the transmitted signal on each of the $N_{R F}$ digital transceivers goes through $N_{t}$ RF paths (mixer, power amplifier, phase shifter, etc.) and summed up before being connected with each antenna see Fig. 4.a. In sub-connected or partially connected structure each of the $N_{R F}$ $\mathrm{RF}$ chain is connected to $N_{t} / N_{R F}$ number of sub-arrays as shown in Fig. 4.b. The fully-connected structure provides full beamforming gain per transceiver but has a high complexity of $N_{R F} \times N_{t}$ RF paths. On the other hand, sub-connected has lower hardware complexity of $N_{t} \mathrm{RF}$ paths at the cost of $1 / N_{R F}$ beamforming gain compared with fully-connected structure. The sub-connected structure is more practical for UEs in uplink because of stringent power constraints at handheld terminals. In general, a smaller transceiver number $N_{R F}$ brings more EE performance improvement with a given $\mathrm{SE}$ reduction. Thus the combination of massive MIMO systems 


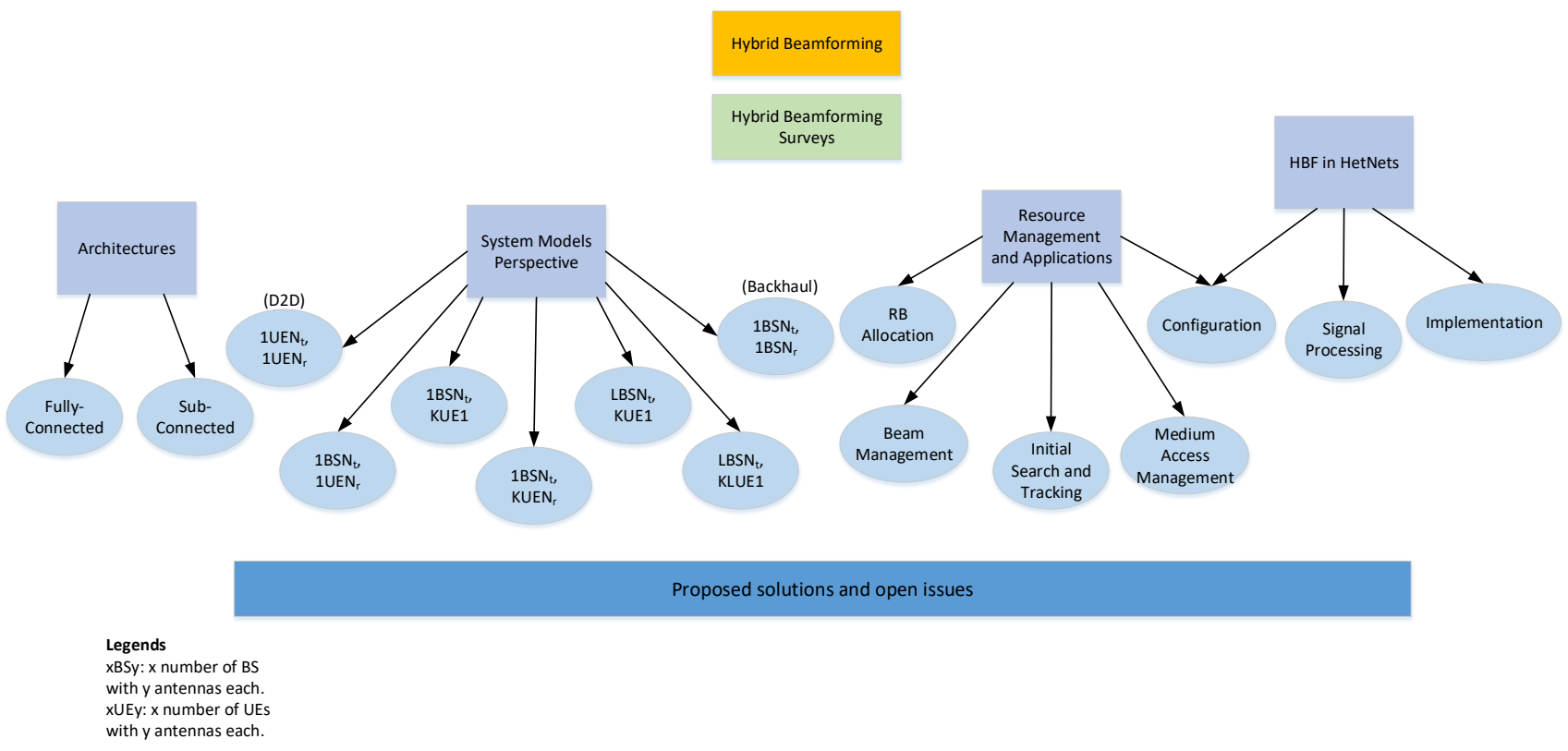

Fig. 2: Taxonomy of hybrid beamforming used in this review paper.

TABLE III: Summary of Review Papers on hybrid beamforming Architectures, MmWave, Massive MIMO, and HetNets/Small Cells

\begin{tabular}{|c|c|c|c|c|c|c|}
\hline & \multicolumn{5}{|c|}{ Hybrid Beamforming Aspects } & General Aspects \\
\hline Paper & Architectures & $\begin{array}{l}\text { MmWave } \\
\text { and/or } \\
\text { massive } \\
\text { MIMO }\end{array}$ & $\begin{array}{l}\text { Digital and } \\
\text { analog BF }\end{array}$ & $\begin{array}{l}\text { Resource } \\
\text { Manage- } \\
\text { ment }\end{array}$ & $\begin{array}{l}\text { HetNets/Small } \\
\text { cells } \\
\text { network }\end{array}$ & Aspects \\
\hline $\begin{array}{l}\text { Molisch et al. } \\
\text { [22] }\end{array}$ & $\checkmark$ & & & & & \\
\hline $\begin{array}{l}\text { Kutty et al. }[23] \\
\text { Zheng } \text { et al. [57] }\end{array}$ & $\checkmark$ & $\checkmark$ & $\checkmark$ & & & $\begin{array}{l}\text { mmWave/massive } \\
\text { MIMO }\end{array}$ \\
\hline Jaber et al. [71] & & & & & & bachhaul \\
\hline Mitra et al. [58] & $\checkmark$ & $\checkmark$ & & & & \\
\hline Roh et al. [59] & $\checkmark$ & $\checkmark$ & & & & \\
\hline $\begin{array}{l}\text { Gupta et al. }[60] \\
\text { Panwar } \text { et al. }[61]\end{array}$ & & $\checkmark$ & & & $\checkmark$ & $\begin{array}{l}\text { CRAN, } \\
\text { D2D, } \\
\text { multi-tier } \\
\text { architectures }\end{array}$ \\
\hline Kim et al. [62] & & & & & & $\begin{array}{l}\text { CRAN, soft- } \\
\text { ware defined } \\
\text { networks }\end{array}$ \\
\hline $\begin{array}{l}\text { Agiwal et al. [63] } \\
\text { Pirinen et al. [64] }\end{array}$ & & $\checkmark$ & $\checkmark$ & $\checkmark$ & & $\begin{array}{l}\text { CRAN } \\
5 \mathrm{G} \text { projects } \\
\text { overview }\end{array}$ \\
\hline $\begin{array}{l}\text { Elijah et al. [66] } \\
\text { Alsharif et al. } \\
\text { [67] } \\
\text { Muirhead et al. } \\
\text { [68] }\end{array}$ & $\checkmark$ & & & & & $\begin{array}{l}\text { mmWave/massive } \\
\text { MIMO } \\
\text { mmWave/massive } \\
\text { MIMO, } \\
\text { HetNets }\end{array}$ \\
\hline $\begin{array}{l}\text { Bogale et al. [47] } \\
\text { Han et al. }\end{array}$ & & $\checkmark$ & $\checkmark$ & & $\checkmark$ & $\begin{array}{l}\text { HetNet/Small } \\
\text { cells network }\end{array}$ \\
\hline Noura et al. [72] & & & & & & $\begin{array}{l}\text { Interference } \\
\text { management } \\
\text { in D2D }\end{array}$ \\
\hline
\end{tabular}




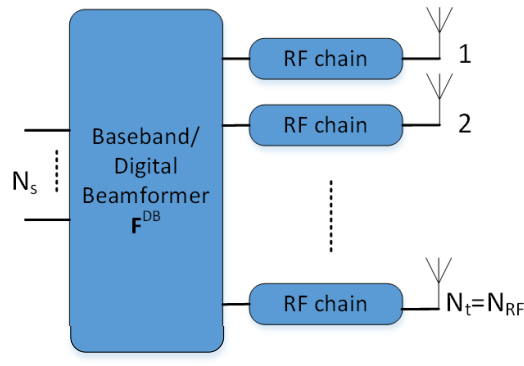

a. Digital Beamforming

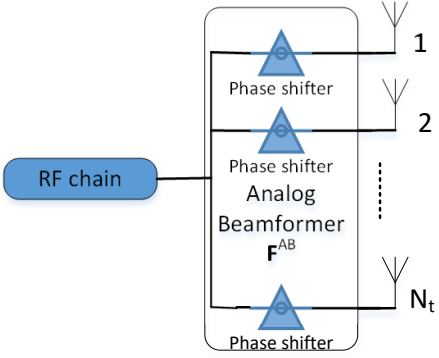

b. Analog Beamforming

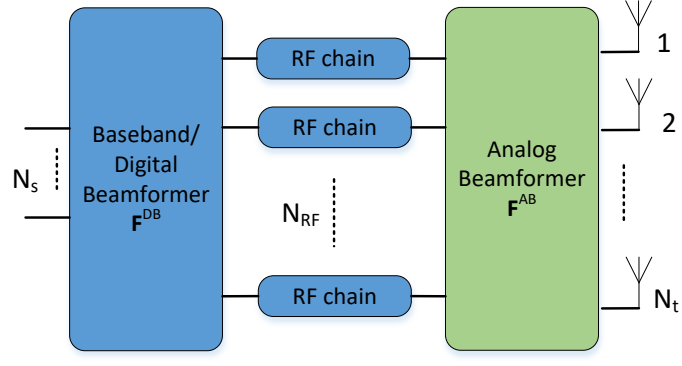

c. Hybrid Beamforming

Fig. 3: Beamforming architectures in mmWave massive MIMO systems: (a) Fully-digital architecture, (b) Analog only architecture, (c) Hybrid analog/digital architecture.

with hybrid beamforming structure including a much reduced transceiver number, is expected to bring significant $\mathrm{EE}$ and $\mathrm{SE}$ enhancement to $5 \mathrm{G}$.

1) Challenges and Performance of Fully-connected hybrid beamforming Architectures: Despite its complexity, the fullyconnected hybrid beamforming architectures are used in several research works to improve the different performance metrics mainly, the EE and SE. For a fully-connected beamforming structure, optimization of the number of transceivers and the number of the antennas per transceiver/RF chain for a fixed total number of antennas $N_{t}$, and for independent numbers $N_{R F}$ and $N_{t} / N_{R F}$ is performed in [26]. It shows optimal points in EE-SE trade-off curves while considering the static power consumption (power dissipated in electronic components) for different number of RF chains and transmit antennas. This could be helpful in choosing different design parameters according to the application requirements. It also shows that hybrid beamforming architectures can archive the multiuser channel capacity. The authors of [52] propose an energy efficient hybrid precoding with a minimum number of $\mathrm{RF}$ chains algorithm and analyze the trade-off between energy and cost efficiency for $5 \mathrm{G}$ wireless communication. This suboptimal solution of BS EE maximization aimed to reduce the energy consumption of RF chains and baseband processing. In the joint optimization of energy and cost efficiency with BS antennas and number of UEs, it shows over $170 \%$ improvement even with the suboptimal solutions. The paper [75] develops an EE based multiuser hybrid beamforming for downlink mmWave massive MIMO systems. The analog beamforming is used to increase the link gain and to select the optimal beam which can maximize the power of the objective user and minimize the interference to all other users. Whereas, the digital beamforming increases the spatial multiplexing gain and maximizes the EE of the objective user with zero-gradientbased approach. Compared to the other works which use traditional MIMO digital beamforming for multiple streams multiplexing-demultiplexing, it uses analog beamforming for the inter-user interference management via beam selection. In [54], [76] the fully-connected hybrid beamforming architecture for a large-scale MIMO system is considered at the TX and the RX. For this structure, considering a limited number of RF chains and finite-resolution PSs, a fast heuristic algorithm is proposed for the case where the number of RF chains $\left(N_{R F}\right)$ is either equal to or greater than the number of data streams $\left(N_{s}\right)$ and showed that the achievable rate can be improved. Because of the non-convex nature of the optimization problem, authors use the constant amplitude assumption on the DB and heuristically solve the $\mathrm{AB}$ problem with per antenna power constraint without complexity analysis. Chiang et al. [77] propose a reduced complexity and feedback overhead method for the joint channel estimation and hybrid beamforming problems. They show that, based on the mmWave channels sparsity and the limited beamwidth, the maximization of the data rates can be obtained by using a channel estimation method to estimate full rank array response matrices [78]. For comparison of the coverage and rate performance of hybrid beamforming enabled multiuser MIMO and single-user spatial multiplexing (SU$\mathrm{SM}$ ) with single-user analog beamforming (SU-BF), a fullyconnected hybrid architecture at the BSs and UEs is considered in [79]. It has been reported that with perfect CSI at the TX and round robin scheduling, MU-MIMO is usually a better choice than SU-SM or SU-BF in mmWave cellular networks. Two precoding/combining strategies taking into account the different hardware constraints, different antenna scales, and different channel characteristics, making them suitable for operation in mmWave systems are presented in [43]. These architectures are the fully-connected hybrid analog/digital precoding/combining and combining with low-resolution ADCs. Both models use fully-connected hybrid beamforming in both TX and RX. In the second model, the RX structure includes a 1-bit ADC used for each in-phase and quadrature baseband received signal. The ADC can be implemented by a single comparator, which results in very low power consumption. This design is suitable for small battery operated UEs in downlink transmission, whereas, the first model can achieve better performance in the backhaul links. The paper [80], proposes hybrid codebooks and precoder designs under the assumption of limited feedback channel between the TX and RX. For the same hybrid beamforming structure, the orthogonal matching pursuit (OMP) algorithm and the gradient pursuit (GP) algo- 


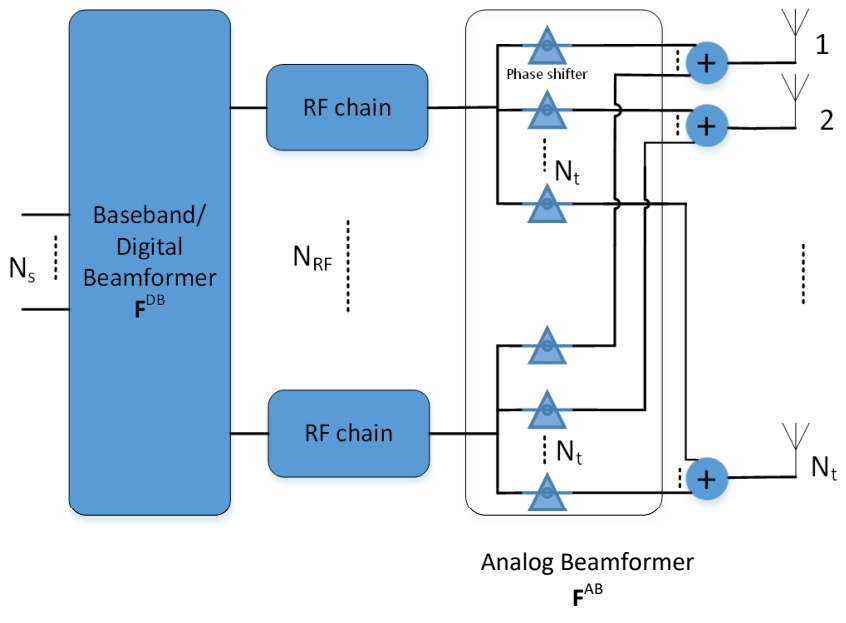

a. Fully connected hybrid beamforming

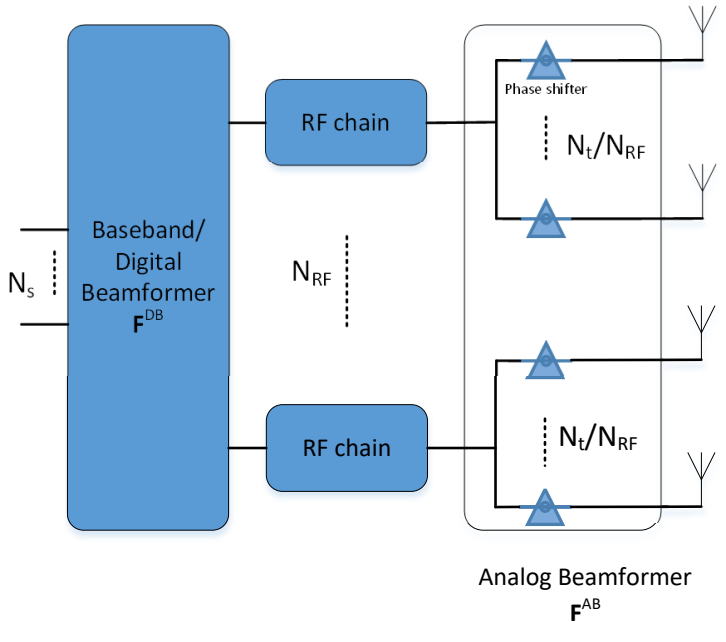

b. Sub-connected hybrid beamforming

Fig. 4: Major types of hybrid beamforming: (a) Fully-connected hybrid beamforming, (b) Sub-connected hybrid beamforming. In the fully-connected hybrid beamforming, each RF chain is connected to $N_{t}$ PSs, hence, there are $N_{R F} N_{t}$ PSs. In the subconnected hybrid beamforming, each RF chain is connected to a subset $\left(N_{t} / N_{R F}\right)$ of PSs, hence, there are $N_{t}$ number of PSs. The fully-connected structure provides gain of $N_{R F} \log _{2}\left(N_{R F}\right)$ in SE over the sub-connected, but at the expense of $N_{R F}$ times more power consumption [26].

rithm are proposed to provide high performance solution to the problem, and to meet the optimization objective [81]. The paper [82] proposes a beam training protocol which effectively accelerates the link establishment while dealing with practical constraints of mmWave transceivers. A geometric approach is used to synthesize multi-beamwidth beam patterns that can be leveraged for simultaneous multi-direction scanning. For a randomly selected single user fully-connected hybrid precoding in mmWave cellular network a simple precoding solution is proposed in [83] assuming only partial channel knowledge at the BS and mobile station in the form of angleof-arrival (AoA). But the proposed solution is limited to single cell and single user. Considering hybrid digital and analog beamforming architecture at the BS and the user terminals with fully-connected hybrid beamforming at both TX and RX, it has been reported in [84] that the same performance of any fully digital beamforming scheme can be obtained with much fewer number of RF chains; the required number of RF chains only needs to be twice the number of data streams. Heuristic algorithms requiring perfect CSI, for overall SE maximization over a point-to-point MIMO system and over a downlink multiusers multiple-input single-output (MU-MISO) system, are proposed when the number of RF chains is less than twice the number of data streams. These algorithms can be modified to be applied when only very low resolution phase shifters are available. To tackle the interference in the mmWave MIMO system under the practical hardware limitation, a hybrid interference cancellation solution tailored for mmWave channel by decomposing the analog beamformer into Kronecker products of unit-modulus component vectors is proposed in [85]. A compressive sensing assisted low- complexity optimal full-digital precoder acquisition algorithm and a beamspace hybrid precoding algorithm for a single-user mmWave MIMO system are proposed in [86]. The beamspace singular value decomposition (SVD) algorithm, is reported to reduce complexity of the hybrid precoding by $99.4 \%$ compared with an optimal full-digital precoder acquisition using fulldimension SVD. However, the real potential of discrete lens array beamspace has not been exploited for the multiuser communication system. In [87], a practical hybrid beamforming for multiuser massive MIMO systems, including ZF precoding in digital beamforming, and beam selection for analog beamforming, is presented. The proposed methods show that the hybrid beamforming with more RF chains can outperform the conventional digital precoding [87]. An iterative hybrid transceiver design algorithm for mmWave MIMO systems [88] shows that it can achieve almost the same performance as the optimal full-baseband design, with much smaller number of RF chains. Based on beam widening with the multi-RF-chain sub-array, two algorithms BWM-MS/LCS and BWM-MS/CF [89] have been proposed to design a hierarchical codebook for channel estimation in mmWave communications with a hybrid precoding structure. It is reported that the algorithms have close performances, and outperform the existing alternatives under the per antenna power constraint. Another enabler of hybrid precoding is the spatial modulation in MIMO systems (SM-MIMO). The fundamental idea of SM-MIMO is in using both the amplitude and phase modulation (APM) and antenna indices to convey information bits with only a single activated antenna. Using the idea of SM-MIMO, the authors of [90], propose an adaptive multimode hybrid precoding for single-RF virtual space modulation with analog phase shift network in 
MIMO systems. The proposed transmission scheme combines the function of analog phase shift network, i.e., the analog precoding, with the SM concept via exploiting the antenna virtualization which allows the RX perceiving virtual antennas instead of physical antennas. The transmission approach includes the analog precoding aided virtual space modulation (APAVSM), and its corresponding multimode hybrid precoder designs in the MIMO system. Evaluation of the proposed designs is achieved through simulations in Rayleigh fading and mmWave channels. An energy-efficiency based multiuser hybrid beamforming for downlink mmWave massive MIMO systems is developed in [75]. The analog beamforming aims to select the optimal beam which can maximize the power of the objective user and mitigate the inter-user interference, whereas, the digital beamforming is solved to maximize the $\mathrm{EE}$ of the BS through flexible baseband processing. For a fully-connected hybrid beamforming, a modeling algorithm is proposed to reduce the computation complexity and minimize the bit-error-rate (BER) to perform precoder/combiner reconstruction [91]. In a fully-connected hybrid beamforming architecture [92], an analytical approach for antenna selection is used to circumvent the degradation in fading channel in conventional antenna selection schemes. This approach requires only variable phase shifters and combiners to reduce the number of RF chains. The presented solution dynamically works both for the diversity as well as the multiplexing gains but obviously with some hardware overhead. The authors of [93], show that in fully-connected hybrid beamforming architecture, both the asymptotic signal-to-interference-plusnoise ratio (SINR) and the effective number of transmit antennas per user can be reduced by a factor of $\pi / 4$. The sumrate degradation can be compensated by using more transmit antennas without any additional RF chains. The author of [94], proposes an iterative hybrid transceiver design algorithm in which the phase shifters can only supply discrete phase adjustments, to maximize the SE and to facilitate low-cost implementation of the analog beamformers via practical finite resolution phase shifters. Another fully-connected architecture, in which the analog beamformer creates multiple 'virtual sectors', which enables separated baseband processing, downlink training, and uplink feedback in different virtual sectors and permit to minimize both signaling overhead and computational complexity is shown in Fig. 5 [22]. This solution can be deployed in the dense urban area with high-rise buildings to facilitate the near LOS acquisition required for the mmWave communications.

2) Challenges and Performance of Partially-connected hybrid beamforming Architectures: The use of multiple antenna arrays for independent beamforming, aimed to obtain diversity/multiplexing gains in mmWave systems. The paper [95] proposes a hybrid beamforming architecture, where the TX and/or RX antenna array consist of multiple subarrays, and each of the subarrays is capable of independent electronic beam steering using RF phase shifters. A multi-beam transmission diversity scheme based on the partial connected hybrid beamforming structure is given in Fig. 4(b). The transmission signal can be adjusted adaptively according to the channel while overcoming the unfavorable channel char-

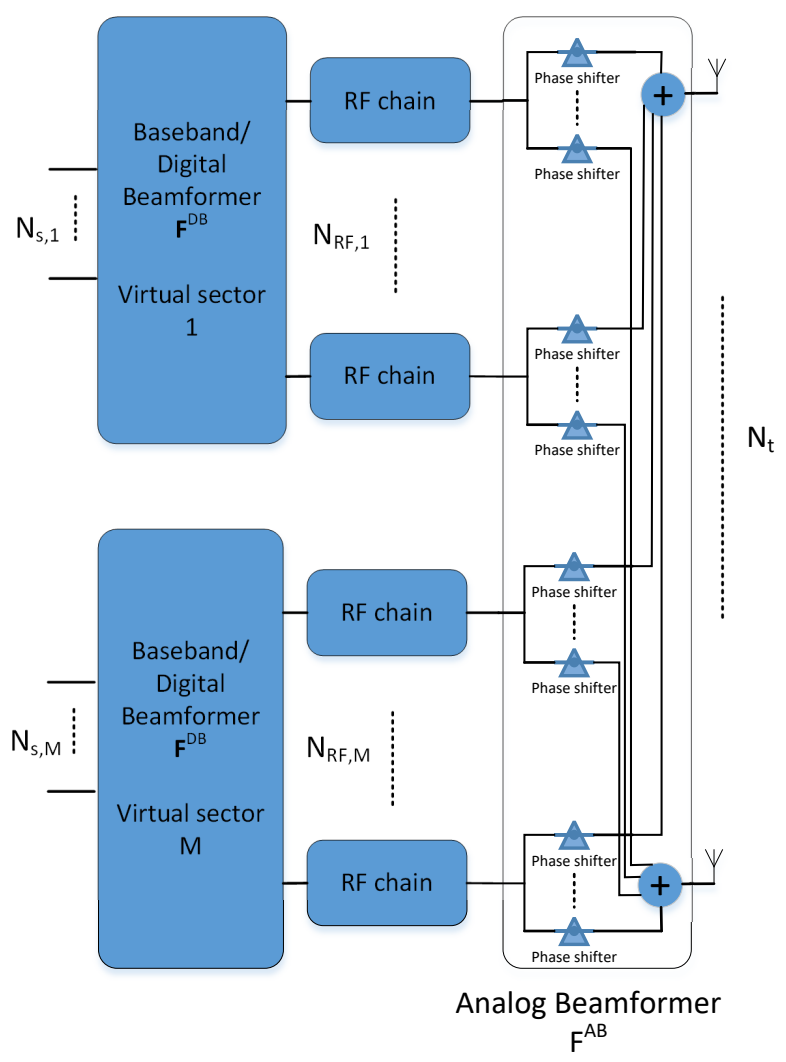

Fig. 5: A fully-connected architecture in which the analog beamformer creates multiple 'virtual sectors' called virtual sectorization. This structure reduces the signaling overhead and computational complexity by providing users' grouping through analog beamforming and serving alike users in the same digital beamformer [22].

acteristics at high frequency bands including mmWave [96]. The sub-connected architecture with multiple steerable beams is practically suitable for the femto-cell BS which could have occasional movement and have to work on batteries (in case of power failure). Among the different hybrid beamforming architectures, the architecture with so-called shared array antenna is analyzed with respect to the peak-to-mean envelope power ratio over antenna elements and the total average transmission power constraint upon multi-beam transmissions [97]. This paper first time proposes a hybrid beamforming solution to well-known peak-to-average-power-ratio (PAPR) problem for massive MIMO-OFDM system. An optimization problem of the analog and digital beamforming matrices aiming to maximize the achievable rate with transmit power constraint is considered in [98]. It also uses a practical TX structure in which each antenna of ULA is only connected to a unique RF chain, and shows that the proposed method outperforms the beam steering method under different configuration of antennas. The authors of [99], propose a hybrid beamforming dedicated to reduce the number of RF chains for single group multicast or broadcast beamforming which has an application in real-time sports broadcast to multiple devices in a same 
building or hall. The combinatorial optimization problem is transformed into a continuous programming formulation by using two bit RF phase shifters as analog beamformers. A low complexity architecture to design the hybrid beamforming system is proposed in [100]. It consists of designing the baseband digital beamforming and then the RF domain analog beamforming via an iterative algorithm. Each antenna of the uniform linear array (ULA) is equipped with a phase shifter. The TX/RX antennas are partitioned into sub-arrays each of which is driven by a digital baseband processing module. An energy efficient design of the hybrid precoder and combiner with sub-connected architecture is presented in [101]. The analog precoder and combiner are optimized via the alternating direction optimization method, where the phase shifter can be easily adjusted with an analytical structure. Then, the digital precoder and combiner are optimized for an effective MIMO communication systems. Rehman et al. [102] propose a mmWave communication system with multiple RF beamforming chains each capable of forming beams in different spatial directions by using iterative search for the capacity maximizing RF beams over subsets of RF chains. Using analog beamforming subarrays such as phased arrays, the hybrid configuration can effectively collect or distribute signal energy in sparse mmWave channels, the multiple digital chains in the configuration provide multiplexing capability and more beamforming flexibility to the system [103]. The analysis of sub-connected architectures reveals that phase shifter based hybrid beamforming gives better performance with narrowband signals, whereas, the tapped delay based beam steering is suitable for wideband signals but suffers from hardware complexity. In [104], a hybrid beamforming structure implemented on dual polarized (DP) planar antenna array (PAA) is presented for downlink MU-MIMO in mmWave channels. An iterative algorithm design permitted to have better SINR than directly steering the RF BFs, and 5-6 dB lower the conventional $\mathrm{BF}$, which uses 8 times more baseband modules for the same $4 \times 4$ DP-PAA. The paper [105], presents the SE performances of different downlink MU-MIMO beamforming strategies for a mmWave communication system using hybrid beamforming assuming perfect knowledge of the CSI at the BS and at the mobile station.

3) Challenges and Performance of Dynamic Sub-array Architecture: A dynamic sub-array structure that selects the subarray according to the long-term channel statistics is proposed in [106] and shown in Fig. 6. As shown in the Fig. 6, the switching matrix is shifted towards the RF chains such that it can select the optimal sub-array along with the phase shifters. For the optimal sub-arrays selection, the exhaustive antenna partitioning has been replaced by a low complexity greedy algorithm that approaches the SE of the optimal exhaustive search solution.

4) Fully-connected and Partially-connected hybrid beamforming Architectures Comparison: The performance loss induced by partially-connected architecture can be compensated by increasing the number of antennas. This constitutes a realistic trade-off between performance loss and the number of phase shifters as compared to the fully-connected architecture [56]. In [107], the hybrid precoder design is

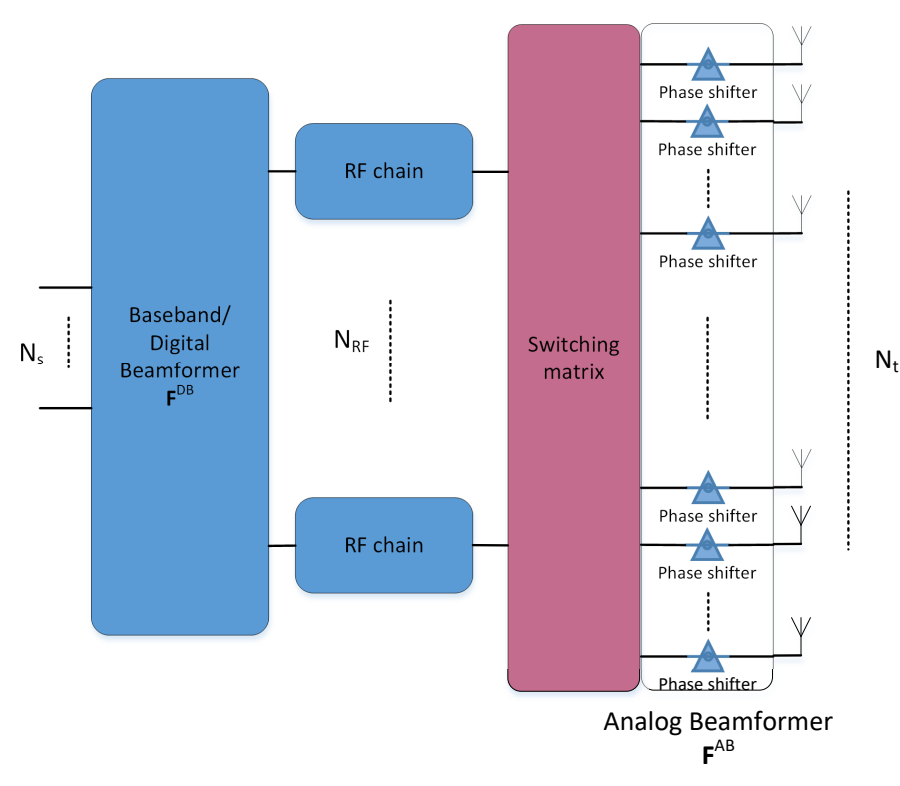

Fig. 6: Hybrid beamforming with dynamic sub-array structure for low complexity sub-array selection. The channel covariance matrix based dynamic antenna sub-array selection can maximize the mutual information rate. It also reduces the power consumption as compared to the PS-based analog precoding/combining. But the current technology of electronic switches at mmWave frequencies is not matured for practical implementation of the switch-based hybrid precoding/combining [106].

considered as a matrix factorization problem. Effective alternating minimization algorithms are proposed for two different hybrid precoding structures, namely the fully-connected and partially-connected structures. This paper shows that the fullyconnected with a higher complexity, cannot approach the performance of the fully digital precoder, unless the number of RF chains is slightly larger than the number of data streams. The partially-connected structure has better performances in terms of SE and provides substantial gains. The authors of [108] propose a successive interference cancellation-based hybrid precoding with near-optimal performance and low complexity. It is noteworthy that partially-connected architecture requires less phase shifters than the fully-connected architecture requires; and therefore, it is more energy efficient. Heuristic algorithms are proposed in [109], for the design of two different hybrid beamforming structures, the fully-connected and partially-connected structures. Such algorithms permit to maximize the overall SE of a broadband system. Besides, the proposed algorithm for the fully-connected structure can achieve SE close to that of the optimal fully-digital solution with much less number of RF chains. An alternating minimization approach for the hybrid precoding design in mmWave MIMO systems under assumption of an averaged channel is proposed in [110]. For the partially-connected structure, the precoder design solution is obtained by using the semi-definite relaxation enabled alternating minimization algorithm. It is remarkable that the fully-connected structure has a higher SE 


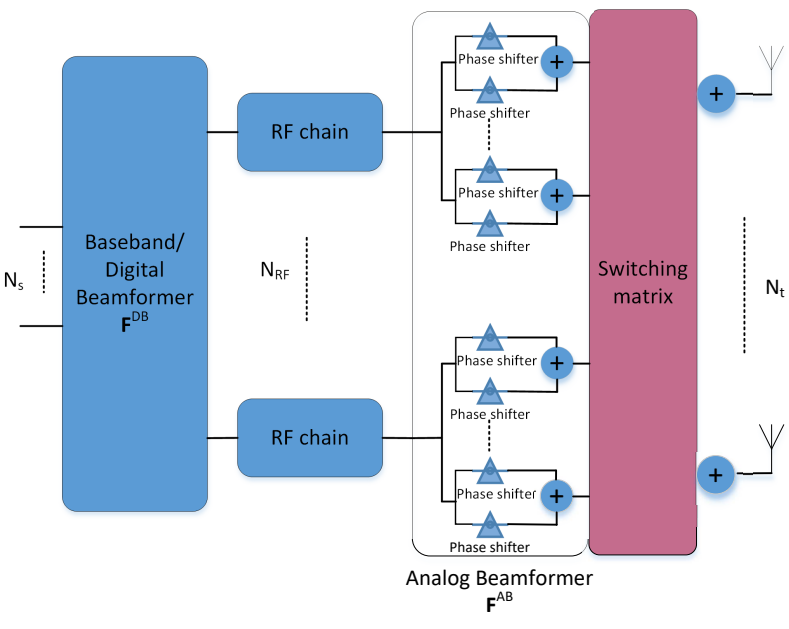

Fig. 7: A sub-connected hybrid beamforming architecture that gives the performance of ideal (pure digital) beamformer with $2 N_{R F}\left(N_{t}-N_{R F}+1\right)$ phase shifters and $N_{R F}$ RF chains [49].

while the partially-connected structure, achieves higher EE.

Antenna selection and hybrid beamforming, are proposed as full digital beamforming alternatives, for simultaneous wireless information and power transfer in a multi-group multicasting scenario in [111]. For antenna selection, only the selected antennas and the corresponding RF chains are active with the help of RF switches. The authors of [112], used antenna selection technique to establish low-complexity algorithm that can jointly determine the RF beamforming array vector and baseband precoding matrix for 2D planar antenna array in mmWave systems. Bogale et al. [49] show that if we realize each element of the analog beamforming matrix by the sum of two phase shifters as shown in Fig. 7, then, the maximum number of nonzero elements in analog beamforming matrix reduces to $N_{R F}\left(N_{t}-N_{R F}+1\right)$. This beamforming structure gives the performance of ideal (pure digital) beamformer with $2 N_{R F}\left(N_{t}-N_{R F}+1\right)$ phase shifters and $N_{R F}$ RF chains. With the help of switching matrix this design can be customized to have either fully-connected or partially-connected hybrid beamforming architecture.

Though mostly hybrid beamforming consists of symmetric precoder at TX and combiner at the RX, there are some works that focus only on the hybrid combiner design. The authors of [113], have explored the performance of two hybrid combining architectures for mmWave based on phase shifters and switches see Fig. 8 . It has been reported that the architecture based on switches is a low power and low complexity solution for standard array sizes and equal number of RF chains. The results show that the switching architecture with the proposed hybrid antenna selection and combining algorithms provides good SEs, close to the one obtained with the architecture based on quantized phase shifters. Six hybrid combining architectures in Fig. 9 are analyzed using algorithms for CSI estimation [44] using phase shifters or switches as main building blocks in the analog processing stage . A generalized hybrid beamforming architecture namely Overlapped Sub-Array
(OSA) structure which includes both fully-connected and subarray architecture is proposed in [114]. Using a Unified Low Rank Sparse recovery algorithm for hybrid beamforming in downlink multiuser scenarios, the OSA design is reported to be a good compromise of the performance and the required hardware complexity. Compared to the existing equal gain transmission (EGT) scheme for the fully-connected array, it is possible to maintain good performance with an around $50 \%$ complexity reduction. Different hybrid beamforming architectures presented in the recent papers are summarized in Table IV. Table VI shows that the fully-connected structure always dominates the other architectures in terms of the SE performance for any combination of $N_{t}, N_{r}$, regardless of the digital/analog signal processing techniques. Fully-connected structure provides approx. $N_{R F} \log _{2} N_{R F}$ b/s/Hz higher SE performance than the sub-connected structure. The power consumption of fully-connected is $N_{R F}$ times higher than the sub-connected. Comparisons of fully-connected and subconnected are provided by [26], [22], [106] and reported in the Table VI. The virtual sectorization architecture in Fig. 5 is a type of fully-connected structure with the separate digital beamformer for each virtual sector. This structure has similar SE performance to that of fully-connected. The dynamic sub-array structure in Fig. 7 uses switches and PSs to dynamically adapt the average channel statistics. Its SE performance is lower than the fully-connected but higher than the sub-connected architectures for all SNR values, number of RF chains, and the number of antennas. Although, the practical implementation of the switches at mmWave frequencies incur high insertion losses and are not feasible with current stateof-the-art technology [113], theoretical comparison shows that there is a large performance gap between the PS-based and switch-based analog beamforming structures. The PS-based architecture is far better than the PS-based structure in SE performance. The performance gap is greater than $2 \mathrm{~b} / \mathrm{s} / \mathrm{Hz}$ in the practical range of RF chains $1-7$. However, switch-based architecture gives $45-70 \%$ reduction in power consumption for equal number of RF chains. The Table VI lists the hybrid beamforming structures for precoders and combiners in the decreasing order of the SE performance along with the usability of each structure for easy comparison. The SE performance of precoder and combiner structures is shown in Fig.10 and Fig. 11, respectively.

\section{B. Phase Shifters, DACs/ADCs Resolutions, Antennas config- urations and technologies}

Hybrid beamforming consists of baseband digital beamforming and the RF analog beamforming. The digital beamforming part contains the power-hungry DAC at the TX side and $\mathrm{ADC}$ at the RX side, whereas, the analog beamforming part contains phase shifters network. The practical circuits are implemented with finite resolution DAC/ADC and phase shifter. At mmWave frequencies, the high sampling rate and high bit-resolution requirements entail high power consumption [43]. As DAC consumes less power compared with its counter-part $\mathrm{ADC}$ (as a reference, a high resolution ADC $\geq$ 8bits consumes several Watts [132]), the most of the 


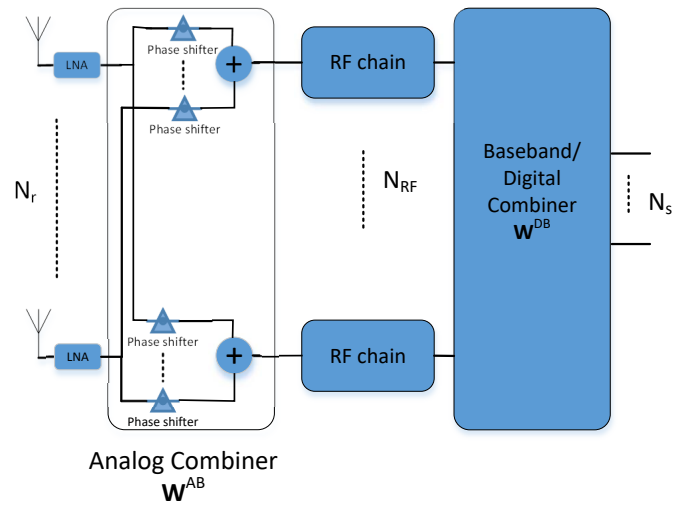

a. Hybrid combiner with phase shifters

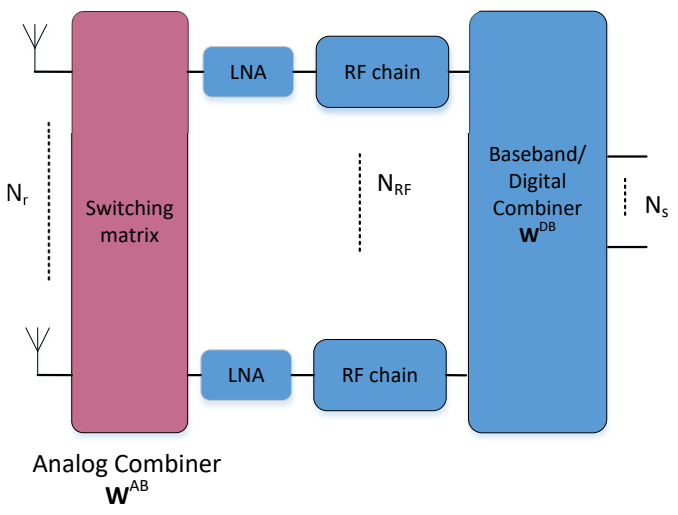

b. Hybrid combiner with antenna selection

Fig. 8: Two different architectures for hybrid combining in MS: (a) Fully-connected hybrid combining with phase shifters, (b) Hybrid combining with antenna selection. Switch-based hybrid combiner consumed $45-75 \%$ less power than the PS-based combiner in the setting of $N_{r}=8-32$ with $N_{R F}=4$ [113].

research on the power and energy minimization focuses on the ADC or PS. There is a lack of research work on joint optimization of ADC, DAC, and PS resolutions. There are many research works that optimize one of them by considering full or infinite resolution for others. Hybrid precoding/combining and pure digital combining with 1-bit ADC resolution are compared in [43]. It shows that at low and medium SNR regimes, the combining with low resolution $\mathrm{ADC}$ has the same performance of hybrid beamforming (which uses fewer RF chains with full precision ADCs). In mmWave massive MIMO systems, both approaches can coexist in the same system. For example, a power-limited mobile station might adopt combining with low-resolution ADCs on the downlink, while hybrid precoding/combining might be used for the uplink [43]. Hybrid beamforming with few bits ADC has been analyzed for SE and EE trade-off in [127], [133], [134].

It has been concluded in [127] that the hybrid combining with coarse quantization (4-5 bits) achieves better SE-EE trade-off compared with both hybrid combining with fullresolutions ADCs and pure digital combining with 1-bit ADC. Roth et al. [133] show that in the low SNR regime the performance of pure digital beamforming with 1-2 bits resolution outperforms hybrid beamforming. However, the hybrid beamforming with 3-5 bits resolution achieves the best ratio of SE and power consumption for the RF RX frontend over a very wide SNR region. Digital, hybrid, and analog combining schemes are compared for SE and EE trade-off in [134] by taking into account all RX's components (not only the ADC). The analog combining achieves both the best SE and EE when the mmWave channel has rank 1 and/or in very low SNR links, and is the only viable architecture under a very stringent power constraint. When comparing the digital combining with fewer bits ADC and the hybrid combining, the digital combining always have better SE performance. It shows that in the downlink where the RX is equipped with a small number of antennas, the digital combining achieves better performance than the hybrid combining, whereas in the Uplink, the hybrid combining outperforms the digital combining in terms of EE. It is shown in [135] that for the massive MU-MIMOOFDM uplink system, the coarse quantization (e.g., four to six bits $\mathrm{ADC}$, depending on the ratio between the number of BS antennas and the number of users) gives no performance loss compared with the infinite-precision ADC by assuming infinite resolution PSs. Ayach et al. [42] show that 2 bits PS yields almost perfect performance for a $64 \times 16$ systems with $N_{s}=1$ and no more than 3 bits are needed to quantize each steering angle in practical systems with full resolution ADC. In a sub-connected architecture, [99] uses 2-bit PS to analytically analyze the multicasting problem. The 2-bit structure transforms the complex combinatorial problem in a continuous optimization form which can be solved by semidefinite programming. Chen et al. [94] propose an iterative algorithm based on the coordinate descent method to design the phases of analog beamformer. The simulation results show that the 2-bit resolution PSs can provide comparable SE to the OMP algorithm with infinite resolution PSs [42], [88], [107]. A 2-bit phase shifter based hybrid scheme is presented in [136]. The quantized RF precoder is combined with the channel matrix to form an equivalent channel and then $\mathrm{ZF}$ baseband precoding is applied to get the hybrid phased ZF (PZF) scheme. The PS and ADC resolutions and their effects are summarized in Table V.

Adaptive control of antenna directivity permits to compensate the radio propagation loss which increases in high frequency bands by using massive-element antennas. In [137], the authors describe the operation and effect of massive element antennas as 5G multi-antenna technology, the related technical issues in high frequency bands and the practical implementations of NTT DOCOMO of this type of antennas. It is reported that using a flat antenna array with a uniform antenna spacing (element spacing equal half the wavelength $(7.5 \mathrm{~mm}))$ in the $20 \mathrm{GHz}$ band, 256 elements can be mounted in an area approximately $12 \mathrm{~cm}^{2}$. The number of mounted elements can be significantly increased when using higher 


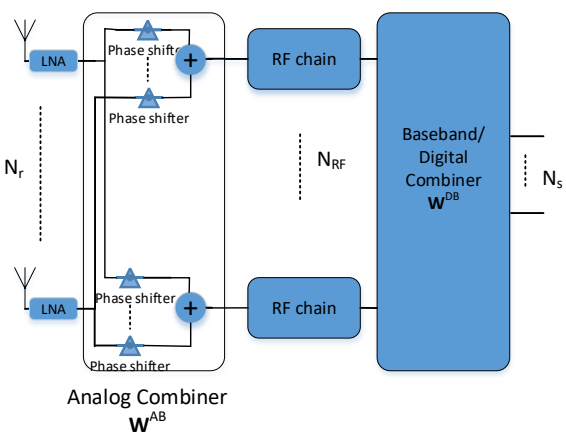

a. Hybrid combiner with phase shifters

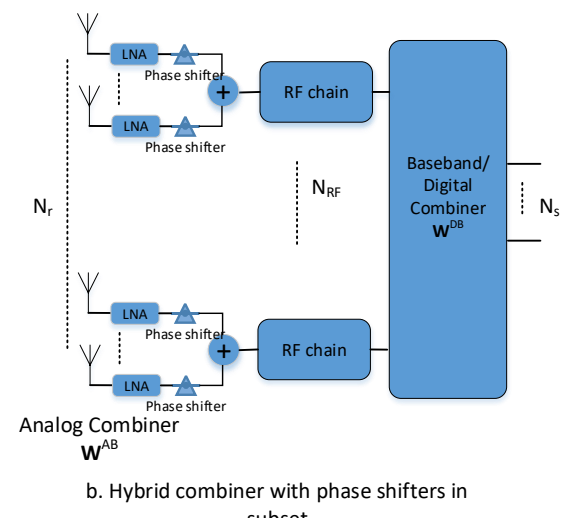

subset

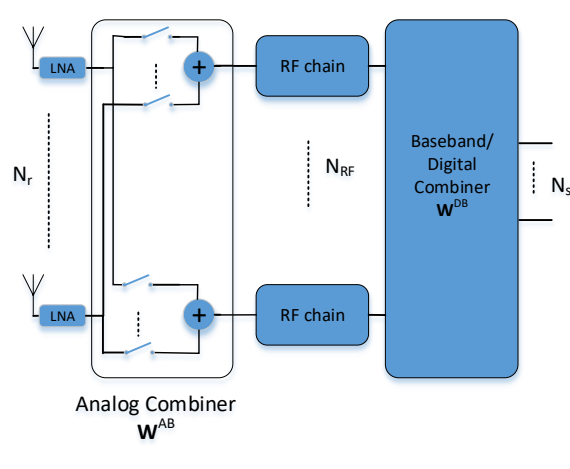

c. Hybrid combiner with switches

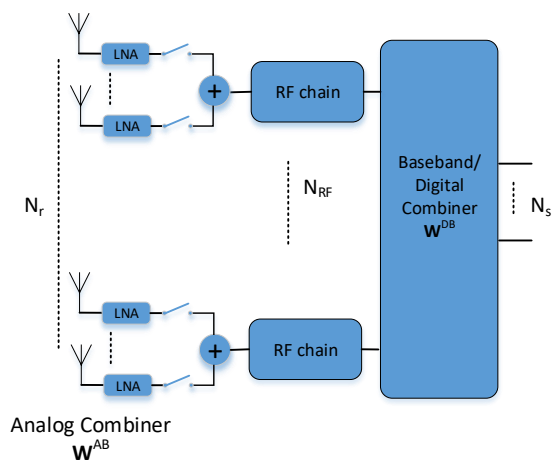

d. Hybrid combiner with switching network in subset

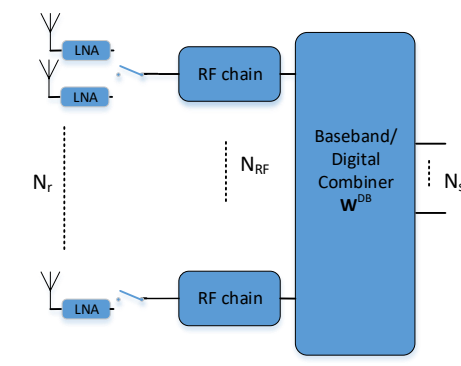

Analog Combiner $\mathbf{W}^{\mathrm{AB}}$

e. Hybrid combiner with switches

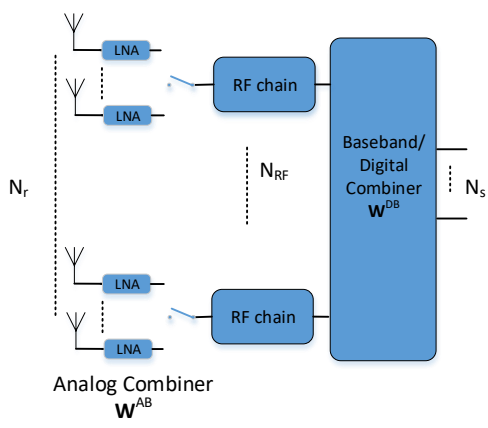

f. Hybrid combiner with switching network in subset

Fig. 9: Hybrid beamforming structures at receive side show better channel estimation performance in case of switch-based combiner. Structures in (a) and (b) are suitable for BS receiver where power and hardware complexity can provide high spectral efficiency. The structures in (e) and (f) are suited for hand-held devices due to the power consumption and hardware complexity constraints at mobile station. These two architectures give low power consumption and low hardware complexity and provide moderate spectral efficiency. Structures in (c) and (d) give marginal improvement over simple antenna selection structures of (e) and (f) [44].

frequency bands [137].

Different configurations of massive MIMO antenna arrays aiming to enhance significantly the performance are described in [138]. Planar antenna arrays obtained by placing linear arrays one parallel to the other, permit to ensure high antenna gains in a desired direction and low side lobe levels in undesired directions. Cylindrical antenna array, a set of piled circular arrays one above the other, provides 360-degree symmetry and increased gain and directivity [138].

Uniform planar array (UPA) antennas structure is used in [120] for mmWave systems. Hybrid beamforming permits to generate beams by combining a multitude of antennas using only a few RF chains.

An integrated antenna is preferred at mmWaves in order to keep the interconnect losses as low as possible. Three main types of technologies are described in [139]. Antennain-Package (AiP): the antenna is integrated in the packaging technology of the IC, which can be of type PCR or LTCC. Antenna-on-Chip (AoC): the antenna is integrated in the backend of an IC, using a monolithic on-chip metalization process. Additional dielectric (superstrate, resonator, lenses) on top of the IC's back-end, permits to improve the off-chip radiation of an antenna. Hybrid integrated mmWave antenna: the antenna is integrated in the same package with the front-end IC, but is neither implemented in the packaging technology nor in the back-end of the chip. The dipole antenna on a fused silica substrate is an example of such technology. The AiP and hybrid approaches are reported to achieve the best radiation efficiency compared to the AoC approach. However, based on some considerations with respect to performance and producibility of the individual antenna concepts, it is recommended to use the diverse technology and approach depending on the target application.

\section{Future research Directions}

The joint optimization of EE-SE [44] could be investigated under the bandwidth, power consumption in RF chain and power consumption in phase shifters and power amplifiers. It would be interesting to see the performance of EE-SE optimization algorithms [52] in the presence of inter-cell and intra-cell interference. In MU massive MIMO systems [43], the large feedback overhead for channel estimation and sparse nature of mmWave channel suggests the use of compressed 
TABLE IV: Hybrid Beamforming Architectures Summary

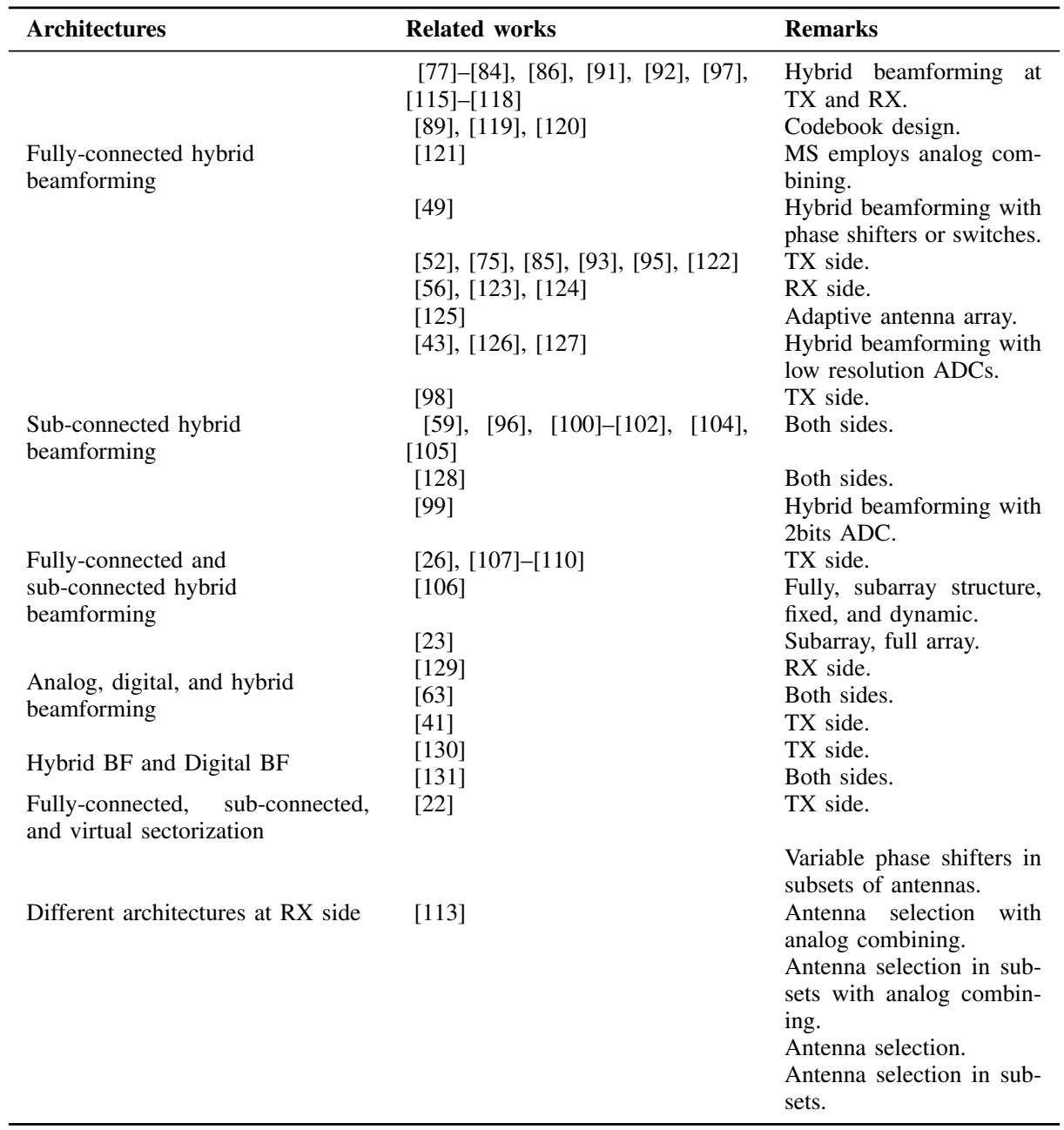

sensing estimation of multiuser mmWave channel for further improvement. The precoding solution with partial channel knowledge in the form of AoA, channel sparsity, reciprocity, and the basis pursuit in [83] can be extended to multi-cell and multiuser scenario. Since AoA estimator uses the best long-term average power directions [95], as a future research, it is possible to reduce the pilot overhead by transmitting the pilot signals for only a subset of the beam pairs at any time instant without impacting the performance of the AoA estimator. Detailed studies in this context are important topics for the future research. In the multi-beam transmission diversity scheme of [96], one can investigate the beam search complexity without the performance loss. The two step approach for hybrid beamforming in sub-connected architecture of [100] can be extended with more practical partial CSI knowledge or with multi-cell multiuser interference. It would be interesting to perform the theoretical analysis of the upper and lower bounds gap between the converging solution and the optimal solution for the RF beam steering iterative search algorithm [102]. The addition of the rank optimization and the multiuser MIMO would also be valuable. The cost comparison of different combinations of hardware components in the hybrid beamforming RX is given in [103, Fig.5], the EE and SE analysis of these architectures would be interesting research contribution. On the radio side, the future research should be focused on successful fabrication and test of a modular array prototype at mmWaves. On the other hand, the radiation efficiency measurement, the effect of packaging on the antenna performance and the yield of the integrated antenna concepts, still need to be investigated in the future.

\section{Hybrid Beamforming: A System Model PERSPECTIVE}

Hybrid beamforming in mmWave massive MIMO systems has been studied by the $5 \mathrm{G}$ research community in various combinations of transmit/receive number of nodes and the number of antennas. In the hybrid beamforming, a signal is first digitally precoded by a baseband precoder, passes through the RF chain (ADC/DAC, data converter, mixer), and then processes in analog phase-only precoder as shown in Fig. 12. Let the digital and analog precoders are denoted by $\mathbf{F}^{D B}$ and $\mathbf{F}^{A B}$, respectively, the corresponding digital and analog combiner are denoted by $\mathbf{W}^{D B}$ and $\mathbf{W}^{A B}$, and $N_{t}$ and $N_{r}$ are the number of transmit and receive antennas, respectively, then the receive signal at the output of the receive combiner is given by

$$
\mathbf{Y}=\mathbf{W}^{D B^{*}} \mathbf{W}^{A B^{*}} \mathbf{H} \mathbf{F}^{A B} \mathbf{F}^{D B} \mathbf{S}+\mathbf{W}^{D B^{*}} \mathbf{W}^{A B^{*}} \mathbf{n}
$$


TABLE V: Hybrid Beamforming Architectures: PS and ADC Resolutions

\begin{tabular}{|c|c|c|c|c|c|c|c|}
\hline Paper & Combining & Phase shifters & ADC resolution ${ }^{1}$ & SE & Power consumption & $\mathbf{E E}$ & Remarks \\
\hline $\begin{array}{l}\text { Alkhateeb et al. } \\
\text { [43] }\end{array}$ & Digital & $\begin{array}{l}\text { Infinite } \\
\text { resolution PS }\end{array}$ & 1-bit & low & lowest & high & \multirow{5}{*}{$\begin{array}{l}\text { Low EE due to } \\
\text { high rate loss. }\end{array}$} \\
\hline \multirow{4}{*}{ Mo et al. [127] } & Hybrid & 7-bit PS & full precision & high & high & low & \\
\hline & Hybrid & $\begin{array}{l}\text { Infinite } \\
\text { resolution PS }\end{array}$ & $1-2$ bits & low & low & low & \\
\hline & Hybrid & $\begin{array}{l}\text { Infinite } \\
\text { resolution PS }\end{array}$ & $4-5$ bits & high & med & high & \\
\hline & Hybrid & $\begin{array}{l}\text { Infinite } \\
\text { resolution PS }\end{array}$ & $7-8$ bits & high & high & low & \\
\hline \multirow[t]{2}{*}{ Roth et al. [133] } & Digital & $\begin{array}{l}\text { Infinite } \\
\text { resolution PS }\end{array}$ & 1-bit & low & lowest & high & \multirow[t]{2}{*}{$\begin{array}{l}\text { Low } \\
\text { regime. }\end{array}$} \\
\hline & Hybrid & $\begin{array}{l}\text { Infinite } \\
\text { resolution PS }\end{array}$ & $3-5$ bits & high & med & high & \\
\hline \multirow[t]{3}{*}{ Abbas et al. [134] } & Hybrid & $\begin{array}{l}\text { Infinite } \\
\text { resolution PS }\end{array}$ & $1-2$ bits & low & low & low & $\begin{array}{l}\text { Suitable for } \\
\text { uplink with } \\
\text { small number of } \\
\text { receive antennas }\end{array}$ \\
\hline & Hybrid & $\begin{array}{l}\text { Infinite } \\
\text { resolution PS }\end{array}$ & $3-5$ bits & high & low & high & $\begin{array}{l}\text { High EE in up- } \\
\text { link. }\end{array}$ \\
\hline & Hybrid & $\begin{array}{l}\text { Infinite } \\
\text { resolution PS }\end{array}$ & full precision & high & high & low & $\begin{array}{l}\text { Suitable for } \\
\text { uplink with } \\
\text { small number of } \\
\text { receive antennas. }\end{array}$ \\
\hline $\begin{array}{l}\text { Studer et al. } \\
{[135]}\end{array}$ & Hybrid & $\begin{array}{l}\text { Infinite } \\
\text { resolution PS }\end{array}$ & $4-6$ bits & \multicolumn{2}{|c|}{$\begin{array}{l}\text { comparable low } \\
\text { to } \mathrm{DC}^{2}\end{array}$} & high & $\begin{array}{l}\text { MU-MIMO- } \\
\text { OFDM uplink. }\end{array}$ \\
\hline Ayach et al. [42] & Hybrid & $2-3$ bits & full precision & \multirow{2}{*}{\multicolumn{2}{|c|}{$\begin{array}{l}\text { comparable } \\
\text { to DC } \\
\text { comparable } \\
\text { to DC }\end{array}$}} & & \multirow{4}{*}{$\begin{array}{l}\text { For single or few } \\
\text { data streams. } \\
\text { When compared } \\
\text { with antenna se- } \\
\text { lection in multi- } \\
\text { casting. } \\
\text { Discrete phases } \\
\text { based on the } \\
\text { coordinate } \\
\text { descent method. } \\
\text { MU-MIMO sys- } \\
\text { tem with quan- } \\
\text { tized phase ZF } \\
\text { precoding. }\end{array}$} \\
\hline Demir et al. [99] & Hybrid & 2-bit & full precision & & & & \\
\hline Chen et al. [94] & Hybrid & 2-bit & full precision & $\begin{array}{l}\text { comparable } \\
\text { to DC }\end{array}$ & & & \\
\hline Liang et al. [136] & Hybrid & 2-bit & full precision & $\begin{array}{l}\text { comparable } \\
\text { to DC } \\
\text { with ZF } \\
\text { precod- } \\
\text { ing }\end{array}$ & & & \\
\hline
\end{tabular}

${ }^{1}$ ADC resolution: Full precision corresponds $\geq 8$ bits

2 DC: Digital Combining

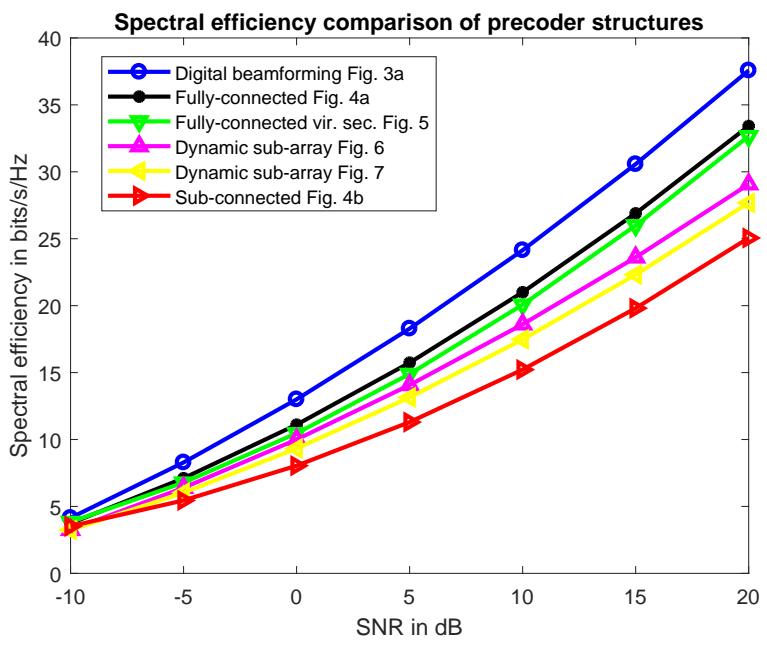

Fig. 10: Spectral efficiency comparison of different precoder structures with $N_{t}=64, N_{r}=4, N_{R F}^{t x}=4, N_{R F}^{r x}=4, K=1$

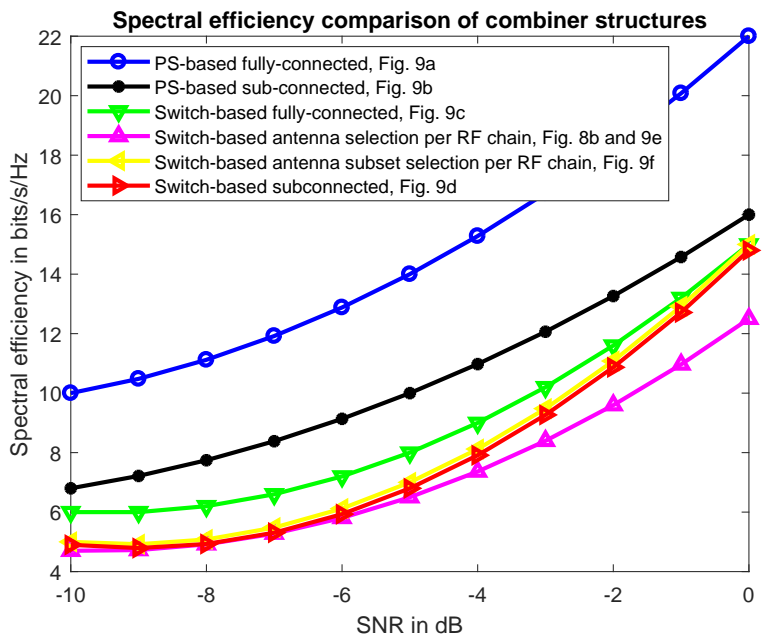

Fig. 11: Spectral efficiency comparison of different combiner structures with $N_{t}=64, N_{r}=16, N_{R F}^{r x}=4, N_{s}=4, K=1$. The transmitter is assumed fully digital, therefore, $N_{R F}^{t x}=64$. 
TABLE VI: Performances of Hybrid Beamforming Structures

\begin{tabular}{|c|c|c|c|c|c|c|c|c|c|c|c|c|}
\hline Papers & Architectures ${ }^{1}$ & $\begin{array}{l}\text { SE } \\
\text { perfor- } \\
\text { mance } \\
(\mathbf{b} / \mathbf{s} / \mathbf{H z}) \\
\end{array}$ & $\begin{array}{l}\text { SNR } \\
\text { (dB) }\end{array}$ & $\begin{array}{l}\text { Performance } \\
\text { gap } \\
(b / \mathrm{s} / \mathrm{Hz})^{2}\end{array}$ & $\mathbf{N}_{\mathbf{t}}$ & $\mathbf{N}_{\mathbf{r}}$ & $\mathbf{K}$ & $\mathbf{N}_{\mathrm{cl}}$ & $\mathbf{N}_{\text {ray }}$ & $\mathbf{N}_{\mathbf{R F}}^{\mathrm{tx}}$ & $\mathbf{N}_{\mathrm{RF}}^{\mathrm{rx}}$ & $\mathbf{N}_{\mathrm{s}}$ \\
\hline \multirow[b]{2}{*}{ Han et al. [26] } & Fully-connected & 28 & -100 & & 500 & 1 & 4 & & & 4 & & \\
\hline & Sub-connected & 20 & -100 & & 500 & 1 & 4 & & & 4 & & \\
\hline Sohrabi et al. [54] & Fully-connected & 9 & -10 & 2 & 64 & 16 & 1 & 1 & 15 & & & \\
\hline Sohrabi et al. [76] & Fully-connected & 6 & -10 & 0.5 & 64 & 8 & 1 & 1 & 20 & & & \\
\hline Chiang et al. [77] & Fully-connected & 0.1 & -10 & & 32 & 32 & 1 & 1 & 8 & 4 & & 2 \\
\hline Chiang et al. [78] & Fully-connected & 3.5 & 10 & & 32 & 32 & 1 & 1 & 5 & 2 & & 2 \\
\hline Alkhateeb et al. [43] & Fully-connected & 5 & -10 & 1 & 64 & 4 & 1 & & & & & 1 \\
\hline Alkhateeb et al. [80] & Fully-connected & 4 & -10 & 1.5 & 32 & 8 & 1 & 6 & 5 & 3 & & 3 \\
\hline Kaushik et al. [81] & Fully-connected & 6 & -10 & 0.2 & 64 & 16 & 1 & 8 & 10 & 4 & & 1 \\
\hline Palacios et al. [82] & Fully-connected & 9 & 10 & & 64 & 24 & 1 & 1 & 3 & 10 & 6 & 1 \\
\hline Alkhateeb et al. [83] & Fully-connected & 7 & -10 & 3 & 200 & 100 & 1 & 1 & 3 & 3 & 3 & 1 \\
\hline Sohrabi et al. [84] & Fully-connected & 10 & -10 & 0.2 & 64 & 16 & 1 & 1 & 15 & 4 & 4 & 4 \\
\hline Zhu et al. [85] & Fully-connected & 25 & 10 & 4 & 16 & 1 & 4 & 1 & 3 & 8 & & \\
\hline Chen et al. [86] & Fully-connected & 5 & -10 & 0 & 64 & 16 & 1 & 1 & 8 & 2 & & 1 \\
\hline Ren et al. [87] & Fully-connected & 5 & -10 & 2 & 100 & 1 & 20 & 1 & 10 & 40 & & \\
\hline Chen et al. [88] & Fully-connected & 8 & -10 & 0.08 & 64 & 16 & 1 & 8 & 10 & 4 & 4 & \\
\hline Xiao et al. [89] & Fully-connected & 4 & -10 & & 32 & 32 & 1 & 1 & 1 & & & \\
\hline Lee et al. [91] & Fully-connected & 8 & -10 & & 8 & 8 & 1 & 1 & 9 & 8 & & 4 \\
\hline Ying et al. [93] & Fully-connected & 8 & -10 & & 128 & 1 & 10 & & & 10 & & \\
\hline Chen et al. [94] & Fully-connected & 15 & -10 & 1 & 144 & 36 & 1 & & 10 & 3 & 3 & 3 \\
\hline Molisch et al. [22] & $\begin{array}{l}\text { Fully-connected } \\
\text { Sub-connected } \\
\text { Fully-connected } \\
\text { (Virtual sec.) }\end{array}$ & $\begin{array}{l}70 \\
25 \\
40\end{array}$ & $\begin{array}{l}10 \\
10 \\
10\end{array}$ & $\begin{array}{l}0 \\
45 \\
30\end{array}$ & 64 & 1 & 16 & 1 & 1 & 16 & & 16 \\
\hline Singh et al. [95] & Sub-connected & 10 & 10 & & $2 \times 8$ & $2 \times 4$ & 1 & & & 2 & 2 & \\
\hline $\mathrm{Xu}$ et al. [98] & Sub-connected & 11 & 10 & & $4 \times 32$ & 4 & 1 & 1 & 1 & 4 & 4 & \\
\hline Wang et al. [100] & Sub-connected & 8 & 10 & & $4 \times 4$ & 16 & 1 & & & 4 & & 1 \\
\hline Park et al. [106] & $\begin{array}{l}\text { Fully-connected } \\
\text { Sub-connected } \\
\text { Dynamic sub-array }\end{array}$ & $\begin{array}{l}22 \\
15 \\
18 \\
\end{array}$ & $\begin{array}{l}10 \\
10 \\
10 \\
\end{array}$ & $\begin{array}{l}3 \\
10 \\
7 \\
\end{array}$ & 64 & 4 & 1 & 8 & 10 & 4 & 4 & \\
\hline Bogale et al. [49] & Sub-connected & 35 & 10 & 15 & 64 & 1 & 8 & 1 & 8 & 16 & & \\
\hline Mendez-Riala et al. [113] & $\begin{array}{l}\text { Switch-based Sub- } \\
\text { connected }\end{array}$ & 0.7 & -10 & & 64 & 16 & 1 & & & & 4 & 4 \\
\hline \multirow{6}{*}{ Mendez-Riala et al. [44] } & $\begin{array}{l}\text { Fully-connected } \\
\text { combiner (DB at } \\
\text { TX) }\end{array}$ & 10 & -10 & & & & & & & & & \\
\hline & $\begin{array}{l}\text { Sub-connected } \\
\text { combiner (DB at } \\
\text { TX) }\end{array}$ & 7 & -10 & & 64 & 16 & 1 & 4 & 6 & 64 & 4 & 4 \\
\hline & $\begin{array}{l}\text { Switch-based fully- } \\
\text { connected combiner } \\
\text { (DB at TX) }\end{array}$ & 6 & -10 & & & & & & & & & \\
\hline & $\begin{array}{l}\text { Switch-based } \\
\text { Sub-connected } \\
\text { combiner(DB at } \\
\text { TX) }\end{array}$ & 4.6 & -10 & & & & & & & & & \\
\hline & $\begin{array}{l}\text { Antennas selection } \\
\text { switches at com- } \\
\text { biner (DB at TX) }\end{array}$ & 5 & -10 & & & & & & & & & \\
\hline & $\begin{array}{l}\text { Antennas selection } \\
\text { switches within the } \\
\text { antenna subsets at } \\
\text { combiner (DB at } \\
\text { TX) }\end{array}$ & 5 & -10 & & & & & & & & & \\
\hline
\end{tabular}

\footnotetext{
${ }^{1}$ Fully-connected architecture in Fig.4(a), sub-connected in Fig.4(b), fully-connected (virtual sectorization) in Fig.5, dynamic sub-array in Fig.6, sub-connected with $2 N_{R F}\left(N_{t}-N_{R F}+1\right)$ PS in Fig.7, switch-based sub-connected combiner in Fig.8, and fully-connected combiner, sub-connected combiner, switch-based fully-connected combiner, switch-based sub-connected combiner, antennas selection switches at combiner, antennas selection switches within the antenna subsets 
TABLE VII: Massive MIMO precoders and combiners in the decreasing order of the SE performance

\begin{tabular}{|c|c|c|}
\hline & Structure & Usability \\
\hline \multirow[t]{5}{*}{ Precoders } & PS-based fully-connected (Fig. 4a) & $\begin{array}{l}\text { Highest SE performance. Suitable for indoor high speed } \\
\text { fixed wireless access points. }\end{array}$ \\
\hline & PS-based fully connected virtual sec. (Fig. 5) & Suitable for FDD system for reduce training overhead. \\
\hline & Dynamic (SW+PS) sub-array(Fig. 6) & $\begin{array}{l}\text { With the current technology switches at mmWave are not } \\
\text { realizable because of high insertion loss [106]. }\end{array}$ \\
\hline & Dynamic (SW+PS) sub-array (Fig. 7) & $\begin{array}{l}\text { With the current technology switches at mmWave are not } \\
\text { realizable because of high insertion loss [106]. }\end{array}$ \\
\hline & PS-based sub-connected (Fig. 4b) & Cellular base-station [26] \\
\hline \multirow[t]{6}{*}{ Combiners } & PS-based fully-connected (Fig. 8a and 9a) & $\begin{array}{l}\text { Highest SE performance. Indoor high speed fixed wireless } \\
\text { access points. }\end{array}$ \\
\hline & PS-based sub-connected (Fig. 9b) & Cellular BS [26]. \\
\hline & Switch-based fully-connected (Fig. 9c) & $\begin{array}{l}\text { Suitable for future BSs with low power consumption } \\
\text { but not realizable with current switching technology at } \\
\text { mmWave [113]. }\end{array}$ \\
\hline & $\begin{array}{l}\text { Switch-based antenna selection per RF chain } \\
\text { (Fig. } 8 \text { b and 9e) }\end{array}$ & $\begin{array}{l}\text { Suitable for future MS with low power consumption } \\
\text { but not realizable with current switching technology at } \\
\text { mmWave [113]. }\end{array}$ \\
\hline & $\begin{array}{l}\text { Switch-based antenna subset selection per RF } \\
\text { chain (Fig. 9f) }\end{array}$ & $\begin{array}{l}\text { Suitable for future MS with low power consumption } \\
\text { but not realizable with current switching technology at } \\
\text { mmWave [113]. }\end{array}$ \\
\hline & Switch-based sub-connected (Fig. 9d) & $\begin{array}{l}\text { Lowest SE performance. Suitable for future low power and } \\
\text { moderate date-rate devices. }\end{array}$ \\
\hline
\end{tabular}

where $\mathbf{H}$ is a $N_{r} \times N_{t}$ complex matrix whose elements are usually modeled by the extended Saleh-Valenzuela model [42], [76], $\mathbf{s}$ is $N_{s} \times 1$ column vector, and $\mathbf{n} \sim C \mathcal{N}\left(0, \boldsymbol{\sigma}^{2}\right)$ is the $N_{r} \times 1$ vector of additive white Gaussian noise (AWGN) of which each element follows complex normal distribution with zero mean and variance $\sigma^{2}$. The signal processing algorithms for $\mathbf{F}^{D B}, \mathbf{F}^{A B}, \mathbf{W}^{D B}$, and $\mathbf{W}^{A B}$ aim to minimize the number of RF chains $\left(N_{R F}\right)$ at $\mathrm{TX}$ and $\mathrm{RX}$ to obtain the optimal performance by exploiting the sparsity of the wireless channel. Within the scope of BS/UE number of antenna configurations, the digital and analog beamforming is also categorized into four classes: codebook design, channel estimation, limited feedback, and low complexity implementation as shown in Table VIII. In the following subsection we classify the hybrid beamforming formulations, solutions, challenges, and the future research directions on the basis of system models.

\section{A. Classification of the Related Work on the Basis of System Models}

1) Hybrid Beamforming: BS with $N_{t}$ antennas and single user with one antenna system $\left(1 B S N_{t}\right.$ and $\left.1 U E 1\right)$ : This system model considers a single cell downlink scenario in which one BS transmits with $N_{t}$ antennas to a single-antenna UE. This is a simplest massive MISO configuration that has been used in few papers for codebook design and performance analysis. The drawbacks of mmWave such as higher pathloss and other atmospheric effects can be ratified by beamforming techniques. However, the beamforming needs CSI, which, in case of mmWave massive MIMO could have large overhead because of large channel matrix. Instead, the beamforming in mmWave systems can be efficiently implemented by using feedback-assisted codebooks [140]. The codebook is designed such that each code vector minimizes the mean square error (MSE) between the code vector's beam pattern and its corresponding ideal beam pattern. Araujo et al. [141] show that massive MISO hybrid beamforming in frequency division duplex (FDD) can provide better SE than the fully digital beamforming in TDD in high mobility scenario. Table IX summarizes the solutions and techniques used to obtain the analog and digital beamforming matrices.

2) Hybrid Beamforming: BS with $N_{t}$ antennas and single user with $N_{r}$ antennas system $\left(1 B S N_{t}\right.$ and $\left.1 U E N_{r}\right)$ : This system model considers a single cell downlink scenario in which one BS transmits with $N_{t}$ antennas to an UE with $N_{r}$ antennas. It is an example of point-to-point massive MIMO system which has been largely investigated in recent research work. In [84], hybrid precoders are designed to maximize the SE for the mmWave massive MIMO systems with single and multiuser cases. Authors show that digital beamforming performance can be achieved if we have twice the number of RF chains than the total number of data streams. Then, they propose heuristic scheme that provides low dimensional baseband precoder and a high dimensional RF precoder, thus reducing the number of RF chains and the power consumption. Ayach et al. [42] use principle of basis pursuit based algorithms to get the unconstrained hybrid RF and BB precoders by solving sparsity constrained matrix reconstruction problem. The low complexity and power efficient design accurately approximates the optimal unconstrained precoders. In contrast to aforementioned works, [83] develops an iterative least square based uplink and downlink precoders to minimize the Frobenius norm between optimal fully digital precoder and the hybrid precoder in the presence of imperfect CSI. The developed precoder has near optimal SE for three simultaneous data streams. Reference [151] combines the beamspace MIMO and hybrid analog-digital transceiver to form continuous aperture phased (CAP) MIMO to achieve near optimal performance. In [107], alternating minimization algorithms are used to get the digital and analog precoders for fullyconnected (in which each RF chain is connected to all the 


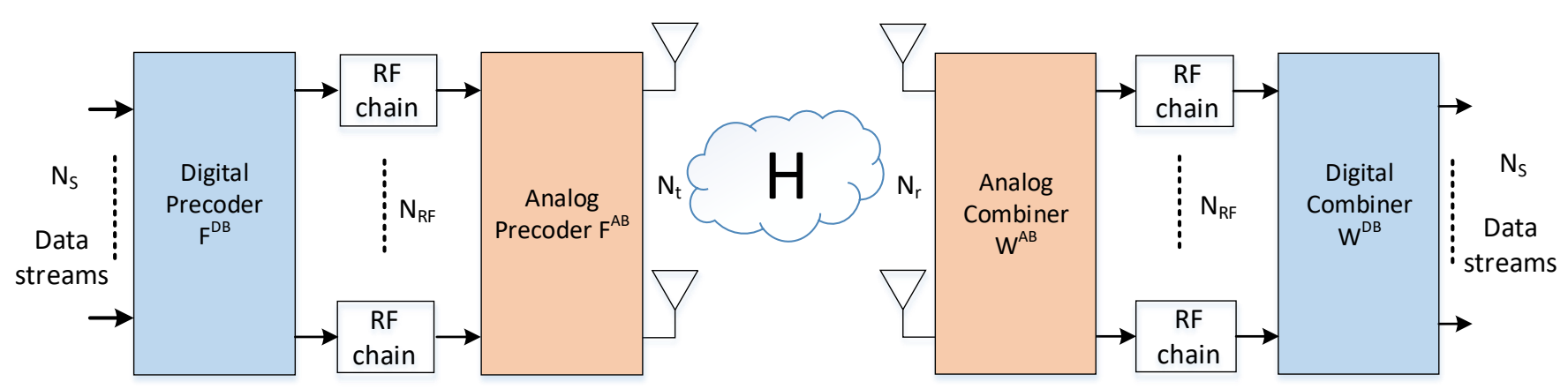

Fig. 12: Block diagram of hybrid precoder and combiner based transmitter and receiver. .

TABLE VIII: Analog and Digital Signal Processing in Hybrid Beamforming Categorized into Four Classes: Codebook Design, Channel Estimation, Limited Feedback, and Low Complexity Implementation

\begin{tabular}{|c|c|c|c|c|c|}
\hline Paper & $\begin{array}{l}\text { Codebook } \\
\text { design }\end{array}$ & $\begin{array}{l}\text { Channel estima- } \\
\text { tion }\end{array}$ & $\begin{array}{l}\text { Limited } \\
\text { feedback }\end{array}$ & $\begin{array}{l}\text { Low complexity } \\
\text { implementation }\end{array}$ & $\begin{array}{l}\text { Energy } \\
\text { efficiency }\end{array}$ \\
\hline Song et al. [140] & $\checkmark$ & & $\checkmark$ & & \\
\hline Araujo et al. [141] & & $\checkmark$ & & & \\
\hline Alkhateeb et al. [83] & & & $\checkmark$ & & \\
\hline Lee et al. [142] & & $\checkmark$ & & & \\
\hline Venugopal et al. [143] & & $\checkmark$ & & & \\
\hline Alkhateeb et al. [117] & $\checkmark$ & $\checkmark$ & & & \\
\hline Chiang et al. [77] & $\checkmark$ & $\checkmark$ & & & \\
\hline Alkhateeb et al. [80] & $\checkmark$ & & $\checkmark$ & & \\
\hline Rajashekar et al. [144] & $\checkmark$ & & & & \\
\hline Rusu et al. [118] & & & & $\checkmark$ & \\
\hline Noh et al. [145] & & & & $\checkmark$ & \\
\hline Zi et al. [52] & & & & & $\checkmark$ \\
\hline Bogale et al. [124] & & $\checkmark$ & & & \\
\hline Liu et al. [146] & $\checkmark$ & & $\checkmark$ & & \\
\hline Jing et al. [75] & & & & & $\checkmark$ \\
\hline Lin et al. [126] & & & & $\checkmark$ & \\
\hline Alkhateeb et al. [147] & & & $\checkmark$ & & \\
\hline Gao et al. [148] & & $\checkmark$ & & & \\
\hline Gao et al. [149] & & $\checkmark$ & & & \\
\hline
\end{tabular}

TABLE IX: Hybrid Beamforming with $1 \mathrm{BS} N_{t}$ and 1UE1

\begin{tabular}{cccc}
\hline Paper & Solution Method & Analog BF matrix & Digital BF matrix \\
\hline Song et al. $[140]$ & MMSE using OMP & Min the residual vector & Generalized Rayleigh quotient [150] \\
Araujo et al. $[141]$ & Eigenmodes selection & SVD & MMSE \\
\hline
\end{tabular}

antenna elements) and partially-connected (the output signal of each RF chain is only connected with $N_{t} / N_{R F}$ antennas) hybrid precoder structures. It treats the hybrid precoding problem as matrix factorization. Joint optimization of $\mathbf{F}^{D B}$ and $\mathbf{F}^{A B}$ is highly complicated due to the element-wise unit modulus constraints of $\mathbf{F}^{A B}$. By decoupling the optimization of these two variables, alternating minimization alternately solves for $\mathbf{F}^{D B}$ and $\mathbf{F}^{A B}$ while fixing the other. Hybrid precoding design in multi-carrier single-user massive MIMO system is presented in [152]. Sparsity of mmWave multi-carrier channel is exploited by using the truncated higher order SVD to compute the RF precoding matrices. Then, the digital precoder is provided by the truncated SVD of the equivalent channel
$\mathbf{H F}^{A B}$. In another method, authors find a low rank unimodular approximation of optimal precoder by $N_{R F}$ times sequentially computing its rank-1 unimodular approximations. In [153], authors consider a hybrid precoding for mmWave massive MIMO systems equipped with linear minimum mean square error (MMSE) equalization. RF precoding is obtained as a solution to a magnitude least square (MLS) approximation problem. It has been shown that the RF precoding design can be approximately transformed into a simultaneous matrix diagonalization problem that leads to an application of known Jacobi algorithms. Channel estimation for mmWave massive MIMO system with a TX with $N_{t}$ antennas and a RX with $N_{r}$ antennas is presented in [142]. The presented channel 
estimation is based on the parametric channel model with quantized AoAs and AoDs. The sparse signal recovery problem is solved by the OMP algorithm employing a redundant dictionary consisting of array response vectors with quantized angle grids. Wideband mmWave channel estimation problem for hybrid architectures is discussed in [143]. The channel estimation problem is formulated as sparse recovery problem and then, compressed sensing based solutions are provided in time, frequency, and combined time-frequency domains. A multi-resolution codebook is designed to obtain beamforming vectors with different beamwidths in mmWave sparse channel [117]. It provides channel estimation for single-path and multipath channels using compressive sensing techniques. Channel estimation without the SVD can be done by orthogonality of the selected array propagation vectors [77]. Thus, the complexity and the feedback overhead can be reduced, because only the codebook indices of the selected array propagation vectors have to be sent to the TX. Another way to reduce the power consumption in mmWave massive MIMO RF chains is the use of antenna selection through electronic switches. Zochmann et al. [154] compare the channel estimation of hybrid beamforming with channel estimation with antenna selection. It has been shown that the antenna selection based channel estimation has a better SE performance than that of the hybrid beamforming based channel estimation due to the limited phase resolution of phase shifters. Complexity and the power consumption can also be reduced by the use of the available inertial measurement units (IMU) in hand-held devices for the receive beamforming as shown in [155]. In this paper, the authors compare the performance of the hybrid beamforming with a fully digital Massive MIMO system, having as many RF chains as the hybrid system, but serving UEs with IMU assisted beamforming abilities. It advocates the use of fully digital beamforming at BS with less number of antennas along with IMU-assisted receive beamforming at users' terminals. In addition to hardware cost and power dissipation in the mmWave massive MIMO systems, one cannot arbitrary increase the number of streams in order to increase the SE because of the limited number of scatterers at mmWave frequencies. Reference [115] provides a guide for the optimal number of data streams which maximizes the SE. It uses a cluster channel model [42] to find the optimal number of data streams, given that $N_{t}, N_{r}, N_{R F}$, the number of clusters and the number of scatterers per cluster. Closed form solutions for hybrid precoding for fully-connected (in which each RF chain is connected to all transmit antennas) and partially connected (in which each RF chain is connected to a specific subset of the antennas) OFDM massive MIMO systems are derived in [106]. Then, a long-term CSI based near optimal adaptive sub-array construction algorithm is designed. In [80], authors develop a codebook that minimizes the average mutual information loss due to the quantized hybrid precoders. Then, they design a greedy hybrid precoding algorithm based on Gram-Schmidt orthogonalization for limited feedback frequency selective mmWave systems. Simulation run-time comparisons for beamforming using perfect CSI, imperfect CSI, ZF precoder based SE, fully digital, fully analog, and hybrid beamforming are provided in [156]. A finite input alphabet (quadrature amplitude modulation constellation) based hybrid beamforming system that maximizes the mutual information is presented in [144]. The optimal solution is achieved by an iterative gradient ascent algorithm that exploits the relationship between the minimum mean-squared error and the mutual information. It also designs a codebook for the analog and digital beamforming/combining matrices based on a vector quantization approach. Hybrid beamforming matrices for frequency-selective mmWave channel are obtained by using time-delay compensation [157]. The designed precoder and combiner maximize the SE and flatten the channel gain.

Four low complexity iterative (alternating optimization) algorithms are provided in [118] for hybrid beamforming in single user massive MIMO system. Authors first design decoupled precoder and study the properties of an optimal unconstrained precoder. Based on these properties, they proposed several low complexity iterative algorithms to obtain the near-optimal analog and digital precoders. Reference [145] proposes a low complexity energy-efficient method for training sequence design based on the steady-state channel estimation and Kalman filtering. The low-dimensionality constraint on training sequence and transmit precoding extends to a hybrid precoding scheme that uses a limited number of active RF chains for transmit precoding by applying the Toeplitz distribution theorem. In a partially-connected hybrid beamforming structure, [131] solves the sum-rate maximization problem iteratively, by taking one RF chain and its connected antenna array at a time. It gives a low complexity suboptimal solution. Reference [97] analyzes the hybrid beamforming partially connected structure with PAPR per subcarrier and total power constraints in massive MIMO-OFDM system. Since the channel estimation depends on the reference signal (RS), a well designed RS is an imperative requirement in massive MIMO system. A beam domain RS design is presented in [158] for partially connected hybrid beamforming structure in lineof-sight (LOS) environment. A consolidated list of reported developments in hybrid beamforming with $1 \mathrm{BS} N_{t}$ and $1 \mathrm{UE} N_{r}$ is presented in Table X.

3) Hybrid Beamforming: BS with $N_{t}$ antennas and $K$ single-antenna UEs $\left(1 B S N_{t}\right.$ and KUE1): This is another widely studied system model in hybrid beamforming signal processing. It represents a point-to-multipoint massive MIMO system where a BS with $N_{t}$ antenna transmits to $K$ singleantenna users. The research works [136] and [49] maximize the SE for $N_{t}$ antenna BS and $K$ single antenna UEs mmWave massive MIMO downlink systems. The optimized hybrid precoders reduce the number of RF chains at the cost of small degradation in the SE performance, specifically in [49] DB performance is obtained with the proposed hybrid beamforming scheme by just utilizing $r_{t} \mathrm{RF}$ chains and $2 r_{t}\left(N_{t}-r_{t}+1\right)$ phase shifters, where $r_{t} N_{t}$ is the rank of the combined digital precoder matrices of all subcarriers. In [159], authors combine the concepts of beamspace MIMO and the beam selection to get the near optimal performance. They evaluate the advantages of the discrete lens array (DLA) based beamspace MIMO scheme via capacity computations, comparisons of the required number of RF chains, and by studying the trade-off between SE and EE. The joint beamspace and beam selection 
TABLE X: Hybrid Beamforming with $1 \mathrm{BS} N_{t}$ and $1 \mathrm{UE} N_{r}$

\begin{tabular}{|c|c|c|c|c|c|}
\hline Paper & Solution Method & Analog BF matrix & Digital BF matrix & RF chains & SE Performance metric \\
\hline $\begin{array}{l}\text { Soharabi et al. } \\
\text { [84] }\end{array}$ & heuristic algorithm & $\begin{array}{l}\text { iterative coordinate de- } \\
\text { scent algorithm }\end{array}$ & $\begin{array}{l}\text { SU: Water-filling solution, } \\
\text { MU: ZF and water-filling }\end{array}$ & $\begin{array}{l}\text { BS: } N_{s} \leq N_{R F} \leq N_{t} \\
\text { UE: } N_{s} \leq N_{R F} \leq N_{r}\end{array}$ & $\begin{array}{l}27 b p s / H z \text { at } 0 d B \text { SNR } \\
\text { with } 64 \times 16, N_{R F}= \\
N_{s}=6 .\end{array}$ \\
\hline Ayach et al. [42] & Basis pursuit & $\begin{array}{l}N_{R F} \text { array response vec- } \\
\text { tors }\end{array}$ & $\begin{array}{l}\text { optimal combination of ar- } \\
\text { ray response vectors }\end{array}$ & $N_{s}<N_{R F}<N_{t}$ & $\begin{array}{l}8.5 \mathrm{bps} / \mathrm{Hz} \text { at } 0 \mathrm{~dB} \text { with } \\
64 \times 16, N_{s}=1, N_{c l}=8, \\
N_{\text {ray }}=10 .\end{array}$ \\
\hline $\begin{array}{l}\text { Alkhateeb et al. } \\
\text { [83] }\end{array}$ & Basis pursuit & $\begin{array}{l}\text { Selected ULA beamsteer- } \\
\text { ing vectors and selected } \\
\text { vectors from quantized } \\
\text { phase shifter matrix }\end{array}$ & $\begin{array}{l}\text { Linear least square esti- } \\
\text { mator }\end{array}$ & $\begin{array}{l}\text { BS: } N_{s} \leq N_{R F} \leq N_{t} \\
\text { UE: } N_{s} \leq N_{R F} \leq N_{r}\end{array}$ & $\begin{array}{l}10.5 \mathrm{bps} / \mathrm{Hz} \text { at } 0 \mathrm{~dB} \text { with } \\
200 \times 100, N_{R F}=3, \\
N_{S}=1 .\end{array}$ \\
\hline Brady et al. [151] & $\begin{array}{ll}\text { Continuous } & \text { Aperture } \\
\text { Phased-MIMO } & \end{array}$ & $\begin{array}{l}\text { Discrete lens array via } \\
\text { Butler matrices }\end{array}$ & $\begin{array}{l}\text { Conventional digital } \\
\text { beamforming for reduced } \\
\text { dimensional orthogonal } \\
\text { beams }\end{array}$ & $N_{R F}<N_{t}$ & $\begin{array}{l}40 \mathrm{bps} / \mathrm{Hz} \text { at } 0 \mathrm{~dB} \text { with } \\
676 \times 676, N_{R F}=4, \\
\text { discrete lens array. }\end{array}$ \\
\hline Yu et al. [107] & $\begin{array}{l}\text { Alternating minimization } \\
\text { (AltMin): Manifold op- } \\
\text { timization, Phase extrac- } \\
\text { tion, semidefinite relax- } \\
\text { ation }\end{array}$ & $\begin{array}{l}\text { Fully connected:Manifold } \\
\text { optimization } \\
\text { AltMin, Fully connected } \\
\text { low complexity: Phase- } \\
\text { extraction } \\
\text { Partially } \\
\text { Semidefinite rennected: } \\
\text { AltMin }\end{array}$ & $\begin{array}{l}\text { Least square solution to } \\
\text { the Euclidean distance be- } \\
\text { tween optimal and hybrid } \\
\text { precoder for fixed analog } \\
\text { precoder }\end{array}$ & $\begin{array}{l}\text { BS: } N_{s} \leq N_{R F} \leq N_{t} \\
\text { UE: } N_{s} \leq N_{R F} \leq N_{r}\end{array}$ & $\begin{array}{l}25 \mathrm{bps} / \mathrm{Hz} \text { at } 0 d B \text { with } \\
144 \times 36, N_{R F}=N_{s}=3, \\
N_{c l}=5, N_{\text {ray }}=10 .\end{array}$ \\
\hline $\begin{array}{l}\text { Zhang et al. } \\
\text { [152] }\end{array}$ & $\begin{array}{l}\text { Higher order SVD, and } \\
\text { Low rank unimodular ap- } \\
\text { prox. }\end{array}$ & Truncated HOSVD & Standard SVD & $\begin{array}{l}N_{R F} \quad<\quad N_{t}, N_{s}= \\
\min \left(N_{R F}, N_{t}\right)\end{array}$ & $\begin{array}{l}160 \mathrm{bps} / \mathrm{Hz} \text { at } 0 \mathrm{~dB} \text { with } \\
256 \times 64, N_{R F}=5, N_{s}= \\
2, N_{\text {ray }}=7 .\end{array}$ \\
\hline Mai et al. [153] & $\begin{array}{l}\text { minimizing the Euclidean } \\
\text { distance between the hy- } \\
\text { brid precoder and the op- } \\
\text { timal minimum MSE pre- } \\
\text { coder }\end{array}$ & $\begin{array}{l}\text { Jacobi algorithm on si- } \\
\text { multaneous matrix diago- } \\
\text { nalization problem }\end{array}$ & $\begin{array}{l}\text { Unconstrained } \\
\text { precoder vaseband } \\
\text { channel }\end{array}$ & $\begin{array}{l}\text { BS: } N_{s} \leq N_{R F} \leq N_{t} \\
\text { UE: } N_{s} \leq N_{R F} \leq N_{r}\end{array}$ & Not available. \\
\hline Lee et al. [142] & $\begin{array}{l}\text { Grid-based Orthogonal } \\
\text { Matching Pursuit for } \\
\text { channel estimation }\end{array}$ & Given Unitary matrix & $\begin{array}{l}\text { Minimize the total coher- } \\
\text { ence of the sensing matrix }\end{array}$ & $N_{R F} \leq \min \left(N_{t}, N_{r}\right)$ & $\begin{array}{l}14 b p s / H z \text { at } 0 d B \text { with } \\
32 \times 32, N_{R F}=2 .\end{array}$ \\
\hline $\begin{array}{l}\text { Venugopa et al. } \\
\text { [143] }\end{array}$ & $\begin{array}{l}\text { Compress sensing based } \\
\text { channel estimation }\end{array}$ & $\begin{array}{l}\text { Phase angles from a given } \\
\text { finite set }\end{array}$ & from a given finite set & $N_{R F} \leq \min \left(N_{t}, N_{r}\right)$ & Not available. \\
\hline $\begin{array}{l}\text { Alkhateeb et al. } \\
\text { [117] }\end{array}$ & $\begin{array}{lr}\text { Adaptive } & \text { compressed } \\
\text { sensing for } & \text { channel } \\
\text { estimation } & \end{array}$ & $\begin{array}{l}N_{R F} \text { array response vec- } \\
\text { tors }\end{array}$ & $\begin{array}{l}\text { optimal combination of ar- } \\
\text { ray response vectors }\end{array}$ & $N_{s}<N_{R F}<N_{t}$ & $\begin{array}{l}15 \mathrm{bps} / \mathrm{Hz} \text { at } 0 d B \text { with } \\
64 \times 32, N_{R F}^{t x}=10, \\
N_{R F}^{r x}=6 .\end{array}$ \\
\hline Chiang et al. [77] & $\begin{array}{l}\text { Compress sensing based } \\
\text { channel estimation and } \\
\text { beamforming }\end{array}$ & $\begin{array}{l}\text { minimize the Frobenius } \\
\text { norm of the error between } \\
\text { the precoder and the right } \\
\text { singular vectors of } \mathrm{H}\end{array}$ & $\begin{array}{l}\text { based on estimated array } \\
\text { propagation vector at TX }\end{array}$ & $N_{R F}<<N_{t}$ & $\begin{array}{l}0.5 \mathrm{bps} / \mathrm{Hz} \text { at } 0 \mathrm{~dB} \text { with } \\
32 \times 32, N_{R F}=4, N_{s}= \\
2, N_{\text {ray }}=8 .\end{array}$ \\
\hline $\begin{array}{l}\text { Zochmann et al. } \\
\text { [154] }\end{array}$ & $\begin{array}{l}\text { Compress sensing based } \\
\text { channel estimation and } \\
\text { beamforming }\end{array}$ & $\begin{array}{l}N_{R F} \text { array response vec- } \\
\text { tors }\end{array}$ & $\begin{array}{l}\text { optimal combination of ar- } \\
\text { ray response vectors }\end{array}$ & $\begin{array}{l}B S: N_{R F}<N_{t}, U E: \\
N_{R F}<N_{r}\end{array}$ & $\begin{array}{l}10 \mathrm{bps} / \mathrm{Hz} \text { at } 0 d B \text { with } \\
64 \times 16, N_{R F}^{t x}=16, \\
N_{R F}^{r x}=8 .\end{array}$ \\
\hline Girim et al. [115] & Basis pursuit & $\begin{array}{l}N_{R F} \text { array response vec- } \\
\text { tors }\end{array}$ & $\begin{array}{l}\text { optimal combination of ar- } \\
\text { ray response vectors }\end{array}$ & $N_{s}<N_{R F}<N_{t}$ & $\begin{array}{l}23 \mathrm{bps} / \mathrm{Hz} \text { at } 12 \mathrm{~dB} \text { with } \\
64 \times 16, N_{R F}=4, N_{s}= \\
2 .\end{array}$ \\
\hline Park et al. [106] & $\begin{array}{l}\text { Closed form solution for } \\
\text { relaxed problem }\end{array}$ & $\begin{array}{l}\text { Optimal solution to the re- } \\
\text { laxed problem }\end{array}$ & $\begin{array}{l}\text { For a given analog pre- } \\
\text { coder and CSI, digital pre- } \\
\text { coder is given by the SVD } \\
\text { of effective channel }\end{array}$ & $N_{R F} \leq N_{t}$ & $\begin{array}{l}7.5 \mathrm{bps} / \mathrm{Hz} \text { at } 0 \mathrm{~dB} \text { with } \\
16 \times 4, N_{R F}=4 .\end{array}$ \\
\hline $\begin{array}{l}\text { Alkhateeb et al. } \\
{[80]}\end{array}$ & $\begin{array}{l}\text { Gram-Schmidt } \\
\text { greedy algorithm }\end{array}$ & $\begin{array}{l}\text { Iterative selection of } \\
N_{R F} \text { beamforming } \\
\text { vectors from given } \\
\text { codebook that maximizes } \\
\text { the mutual information }\end{array}$ & $\begin{array}{l}\text { Based on the selected ana- } \\
\text { log precoder and SVD of } \\
\text { effective channel in }[80 \text {, } \\
\text { eq. (18)] }\end{array}$ & $N_{s}<N_{R F}<N_{t}$ or $N_{r}$ & $\begin{array}{l}10 \mathrm{bps} / \mathrm{Hz} \text { at } 0 d B \text { with } \\
32 \times 16, N_{R F}=3, N_{s}= \\
2, N_{\text {ray }}=6 .\end{array}$ \\
\hline Phyo et al. [156] & $\begin{array}{l}\text { Zero forcing beamforming } \\
\text { algorithm }\end{array}$ & $\begin{array}{l}\text { Monte-Carlo simulation } \\
\text { for rate maximization }\end{array}$ & $\begin{array}{l}\text { Monte-Carlo simulation } \\
\text { for rate maximization }\end{array}$ & $\begin{array}{l}\text { BS: } N_{R F}<N_{t} \text {, UE: } \\
N_{R F}<N_{r x}\end{array}$ & $\begin{array}{l}20 \mathrm{bps} / \mathrm{Hz} \text { at } 0 \mathrm{~dB} \text { with } \\
120 \times 60, N_{R F}^{t x}=16, \\
N_{R F}^{r}=8, N_{\text {ray }}=3 .\end{array}$ \\
\hline $\begin{array}{l}\text { Rajashekar et al. } \\
\text { [144] }\end{array}$ & $\begin{array}{l}\text { Iterative gradient ascent } \\
\text { algorithm }\end{array}$ & $\begin{array}{l}\text { DFT-based directional } \\
\text { codebook }\end{array}$ & $\begin{array}{l}\text { gradient-ascent based iter- } \\
\text { ative solution }\end{array}$ & $N_{s}=N_{R F} \leq N_{t}$ & $\begin{array}{l}8 \mathrm{bps} / \mathrm{Hz} \text { at } 0 \mathrm{~dB} \text { with } \\
32 \times 32, N_{R F}=4\end{array}$ \\
\hline Wang et al. [157] & Time-delay compensation & Iterative path elimination & Water-filling solution & $\begin{array}{l}N_{R F} \text { is a optimization } \\
\text { variable }\end{array}$ & $\begin{array}{l}17 \mathrm{bps} / \mathrm{Hz} \text { at } 0 \mathrm{~dB} \text { with } \\
16 \times 16 \text {. }\end{array}$ \\
\hline Rusu et al. [118] & $\begin{array}{l}\text { Iterative solutions (alter- } \\
\text { nate optimization, } \mathbf{F}^{A B} \text {, } \\
\mathbf{F}^{D B} \text { ) }\end{array}$ & $\begin{array}{l}\text { Minimize MSE between } \\
\text { optimal precoder and ana- } \\
\text { log precoder for fixed dig- } \\
\text { ital precoder }\end{array}$ & $\begin{array}{l}\text { orthonormal Procrustes } \\
\text { problem or least square }\end{array}$ & $N_{s} \leq N_{R F} \leq N_{t}$ & $\begin{array}{l}34 \mathrm{bps} / \mathrm{Hz} \text { at } 0 \mathrm{~dB} \text { with } \\
128 \times 128, N_{R F}=4, \\
N_{s}=2, N_{c l}=3, \\
N_{\text {ray }}=18 .\end{array}$ \\
\hline Dai et al. [131] & Subarray-wise Iterative & $\begin{array}{l}\text { MMSE to obtain precoder } \\
\text { and then matrix decompo- } \\
\text { sition }\end{array}$ & $\begin{array}{l}\text { MMSE to obtain precoder } \\
\text { and then matrix decompo- } \\
\text { sition }\end{array}$ & & $\begin{array}{l}4 \mathrm{bps} / \mathrm{Hz} \text { at } 10 \mathrm{~dB} \text { with } \\
128 \times 16, N_{R F}=16, \\
N_{\text {ray }}=10 .\end{array}$ \\
\hline Kim et al. [97] & Shared array antenna & $\begin{array}{l}\text { Selection from quantized } \\
\text { codebook }\end{array}$ & $\begin{array}{l}\text { Selection from quantized } \\
\text { codebook }\end{array}$ & $\begin{array}{l}N_{R F}<N_{t}, 1 \leq N_{s} \leq \\
\min \left(N_{R F}, N_{t}\right)\end{array}$ & Not available. \\
\hline
\end{tabular}


are used to get the near-optimal performance. The beam selection can be performed according to different parameters, such as the pathloss, the SINR at the RX, the capacity of the system, and the minimum error rate. The mmWave massive MIMO systems increase the capacity of $5 \mathrm{G}$ wireless networks. However, the large number of antennas require separate RF chains, which increases the cost and energy consumption in massive MIMO systems. In [52], authors formulate an EE optimization problem for large number of antennas and RF chains. Suboptimal solution for EE is obtained by separate algorithms for hybrid precoding, minimum RF chains, critical number of antennas, and optimal number of UEs. The cost and energyefficiency of mmWave massive MIMO are addressed by four different angles in [52]. Due to the nonconcave optimization problem, the following suboptimal iterative algorithms are developed: energy-efficient hybrid precoding (EEHP), critical number of antennas searching (CNAS) and user equipment number optimization (UENO) for maximizing the $\mathrm{EE}$ of $5 \mathrm{G}$ networks; and EEHP with the minimum number of RF chains (EEHP-MRFC) to reduce the cost of RF circuits. Cai et al. [160] present a low complexity precoder by implementing the analog beamformer with linear precoder ( $\mathrm{ZF}$ and MRT), namely, ZF-hybrid and MRT-hybrid to control the phase of precoder. It has been shown that at low SNR regime, MRThybrid has better precoding gain and at high SNR values, ZFhybrid precoding scheme achieves near optimal performance. In case of multiple UEs with frequency selective fading in sub $6 \mathrm{GHz}$ frequency bands, a unified analog precoder based on the spatial covariance matrix (SCM) knowledge of all UEs is proposed in [161]. This work is contrary to [160] and it realizes digital precoder matrix by $\mathrm{ZF}$ precoder and the analog precoding matrix is given by $N_{t} \times N_{R F}$ sub-matrix of a $N_{t} \times N_{t}$ unitary matrix with each nonzero element having the same amplitude. In the presence of perfect channel covariance matrix, [124] utilizes the TDD MMSE based hybrid analogdigital estimated channels to exploit beamforming for data transmission. It shows that there is a trade-off between the training duration and achievable throughput when the hybrid analog-digital channel estimation and beamforming is applied with limited number of RF chains. In the hybrid beamforming, the cost and hardware complexity are reduced by connecting one RF chain to multiple antennas. For a MU-MIMO scenario, one RF chain per single-antenna user has been used in [93] to evaluate the performance degradation. It has been shown that the asymptotic SINR is lowered by $\pi / 4$ which can be compensated by $27 \%$ increase in transmit antennas. The CSI impact on the capacity of MU-MIMO is studied in [146]. The analog precoder is selected from the finite codebook. The codebook is designed with instantaneous CSI and hybrid (statistical and instantaneous) CSI at the BS. In hybrid CSI, BS has the knowledge of the distribution of channel and utilizes this information to find the analog precoder. Then, the corresponding digital precoder is obtained from the instantaneous CSI and effective channel $\mathbf{H F}^{A B}$. It is shown that full instantaneous CSI does not help in reducing the RF chains to achieve the first order massive MIMO gain, therefore, low complexity statistical CSI precoding is preferable for practical situations.
The interleaved subarray structure can be used for space division multiplex access in multiuser massive MIMO system because of fine narrow beam formation [162]. The disadvantage of extra inter-antenna elements distance results in grating lobes (side lobes). A robust hybrid beamforming scheme is presented in [162] that can withstand in the presence of AoA estimation error at BS. Common beam is formed through multiple subarrays in order to minimize the maximum difference between estimated user's direction and main beam (main lobe) direction. A consolidated list of reported developments in hybrid beamforming with $1 \mathrm{BS} N_{t}$ and $K \mathrm{UE} 1$ is presented in Table XI.

4) Hybrid Beamforming: BS with $N_{t}$ antennas and $K$ UEs with $N_{r}$ antennas per $U E\left(1 B S N_{t}\right.$ and $\left.K U E N_{r}\right)$ : The considered system model consists of a BS equipped with $N_{t}$ antennas transmitting to $K$ users each with $N_{r}$ antennas. This is a common scenario in a downlink of single cell multiuser massive MIMO system. Usually, a UE is equipped with single RF chain to minimize the signal processing power consumption, whereas $N_{r}$ antennas are used to provide beamforming gain. The high propagation loss at mmWave frequencies can be compensated by the use of antenna arrays and beamforming techniques. Beamforming for multiple users, also known as multiuser precoding, can be utilized to further improve the SE of mmWave MU-MIMO systems. The authors in [163] design a hybrid MMSE multiuser precoder in mmWave massive MIMO system in which a BS, equipped with $N_{t}$ antennas and $N_{R F}$ RF chains, communicates with $K$ remote UEs. Each UE is equipped with $N_{r}$ receive antennas and only one RF chain. They use orthogonal matching pursuitbased algorithm to obtain near optimal SE performance. An energy-efficient hybrid beamforming for mmWave massive MU-MIMO downlink is presented in [75]. This paper proposes the analog beamforming to select the optimal beam which can maximize the power of the objective user and minimize the interference to all other users. In addition, the digital beamforming maximizes the $\mathrm{EE}$ of the objective user with zero-gradient-based approach. It is shown that, in mmWave multiuser massive MIMO system, the analog beamforming can mitigate the inter-user interference more effectively with the selection of the optimal beam. A low complexity hybrid analog beam selection and digital precoder design is proposed in [126]. Based on the CSI from UEs, BS finds the optimal beam subset via alternative tree search algorithm. The proposed solution uses one-bit ADC which results in a low sum-rate. In MU-MIMO, the CSI feedback constitutes a considerable overhead. A limited feedback hybrid precoding scheme is presented in [147]. The formulated problem maximizes the sum rate constraint to the total power and the quantized analog precoders. The mixed integer programming problem is solved in two stages. In the first stage, RF precoder at the $\mathrm{BS}$ and RF combiner at the UE are jointly designed to maximize the desired signal power, and in the second stage, the $\mathrm{BS}$ digital precoder is designed to manage the inter-users interference. Garcia et al. [164] decompose the fully connected hybrid beamforming structure into banks of commonly used RF chains and model their losses using S-parameters. They reveal that insertion losses have a significant impact on the 
TABLE XI: Hybrid Beamforming with $1 \mathrm{BS} N_{t}$ and $K \mathrm{UE} 1$

\begin{tabular}{|c|c|c|c|c|c|}
\hline Paper & Solution Method & Analog BF matrix & Digital BF matrix & RF Chains & SE performance metric \\
\hline Liang et al. [136] & Phased-ZF & $\begin{array}{l}\text { Conjugate transpose of the } \\
\text { aggregate downlink chan- } \\
\text { nel }\end{array}$ & ZF on effective channel & $N_{R F}=K$ & $\begin{array}{l}20 \mathrm{bps} / \mathrm{Hz} \text { at } 0 \mathrm{~dB} \text { with } \\
N_{t}=128, K=4\end{array}$ \\
\hline Bogale et al. [49] & $\begin{array}{l}\text { Independent design of } \\
\text { analog (PS) and digital } \\
\text { (ZF) precoders }\end{array}$ & $\begin{array}{l}\text { Matrix with each element } \\
\text { composed of the sum of } \\
\text { two digitally controlled } \\
\text { phase shifters (PSs) }\end{array}$ & $\begin{array}{l}\text { SVD of ZF precoder and } \\
\text { then truncation for re- } \\
\text { quired number of RF } \\
\text { chain }\end{array}$ & $\begin{array}{l}N_{R F}=r_{t} \leq N_{t}, r_{t}= \\
\operatorname{rank}\left(F^{D B}\right)\end{array}$ & $\begin{array}{l}12 \mathrm{bps} / \mathrm{Hz} \text { at } 0 \mathrm{~dB} \text { with } \\
N_{t}=64, K=8 .\end{array}$ \\
\hline $\begin{array}{l}\text { Amadori et al. } \\
{[159]}\end{array}$ & $\begin{array}{l}\text { Beamspace MIMO and } \\
\text { beam selection }\end{array}$ & $\begin{array}{l}\text { Beam selection algorithms } \\
\text { with discrete lens array }\end{array}$ & $\begin{array}{l}\mathrm{K} \text { dimensional ZF pre- } \\
\text { coder }\end{array}$ & $N_{R F}=K$ & $\begin{array}{l}1.3 \mathrm{bps} / \mathrm{Hz} \text {, at } 0 \mathrm{~dB} \text { with } \\
N_{t}=81 \text { discrete lens ar- } \\
\text { ray, selected antenna sub- } \\
\text { set } N=40, K=40 .\end{array}$ \\
\hline $\mathrm{Zi}$ et al. [52] & $\begin{array}{l}\text { Minimize the Euclidean } \\
\text { distance between optimal } \\
\text { precoder and hybrid pre- } \\
\text { coder }\end{array}$ & $\begin{array}{l}\text { iterative algorithms to } \\
\text { find } N_{R F} \text { column vectors } \\
\text { from steering matrix }\end{array}$ & $\begin{array}{l}\text { Iterative algorithm to find } \\
N_{R F} \text { rows matrix }\end{array}$ & $K<N_{R F}<N_{t}$ & $\begin{array}{l}35 \mathrm{bps} / \mathrm{Hz} \text { at } P_{t}= \\
33 \mathrm{dBm} \text { with } N_{t}=200 \\
K=N_{R F}=10, L=30\end{array}$ \\
\hline Cai et al. [160] & Linear precoding & ZF/MRT precoding & $\begin{array}{l}\text { ZF/MRT precoding on ef- } \\
\text { fective channel }\end{array}$ & $N_{R F}=K$ & $\begin{array}{l}7 \mathrm{bps} / \mathrm{Hz} \text { at } 0 \mathrm{~dB} \text { with } \\
N_{t}=128, K=N_{R F}= \\
8 .\end{array}$ \\
\hline Zhu et al. [161] & $\begin{array}{l}\text { Subspace construction al- } \\
\text { gorithm }\end{array}$ & $\begin{array}{l}\text { Spatial covariance matrix } \\
\text { based }\end{array}$ & ZF precoding & $N_{s}<N_{R F}<<N_{t}$ & $\begin{array}{l}45 \mathrm{bps} / \mathrm{Hz} \text { at } 0 \mathrm{~dB} \text { with } \\
N_{t}=16 \times 16 \text { planar array, } \\
N_{R F}=64, K=16 .\end{array}$ \\
\hline $\begin{array}{l}\text { Bogale et al. } \\
{[124]}\end{array}$ & MMSE & $\begin{array}{l}\text { First } N_{R F} \text { columns of } \\
\text { left singular matrix of } \\
\text { SVD(CE) }\end{array}$ & Interior point method & $N_{R F}<N_{t}$ & Not available. \\
\hline Ying et al. [93] & Eigen-beamforming & $\begin{array}{l}\text { Phase-only eigen- } \\
\text { beamforming matrix }\end{array}$ & Scaled identity matrix & $K=N_{R F}<<N_{t}$ & $\begin{array}{l}35 \mathrm{bps} / \mathrm{Hz} \text { at } 0 d B \text { with } \\
N_{t}=128, K=N_{R F}= \\
10 .\end{array}$ \\
\hline Liu et al. [146] & $\begin{array}{l}\text { Codebook-based hybrid } \\
\text { beamforming }\end{array}$ & $\begin{array}{l}\text { Semidefinite relaxation } \\
\text { and fairness-aware greedy } \\
\text { algorithm }\end{array}$ & $\begin{array}{l}\text { Linear precoding on effec- } \\
\text { tive channel }\end{array}$ & $K=N_{R F}<<N_{t}$ & $\begin{array}{l}42 \mathrm{bps} / \mathrm{Hz} \text { at } 10 \mathrm{~dB} \text { with } \\
N_{t}=64, K=N_{R F}= \\
10 .\end{array}$ \\
\hline Fujio et al. [162] & $\begin{array}{l}\text { Interleaved subarray } \\
\text { beamforming }\end{array}$ & $\begin{array}{l}\text { Weighted array manifold } \\
\text { vectors }\end{array}$ & $\begin{array}{l}\text { Linear precoding on effec- } \\
\text { tive channel }\end{array}$ & & $\begin{array}{l}5 b p s / H z \text { at } 0 d B \text { with } \\
N_{t}=16, K=2 .\end{array}$ \\
\hline $\begin{array}{l}\text { Soharabi et al. } \\
{[84]}\end{array}$ & heuristic algorithm & $\begin{array}{l}\text { iterative coordinate de- } \\
\text { scent algorithm }\end{array}$ & $\begin{array}{l}\text { SU: Water-filling solution, } \\
\text { MU: ZF and water-filling }\end{array}$ & $\begin{array}{l}\text { BS: } K N_{s} \leq N_{R F} \leq N_{t}, \\
\text { UE: } N_{s} \leq N_{R F} \leq N_{r}\end{array}$ & $\begin{array}{l}26 \mathrm{bps} / \mathrm{Hz} \text { at } 0 d B \text { with } \\
N_{t}=64, K=8, N_{R F}= \\
9 .\end{array}$ \\
\hline
\end{tabular}

EE of the hybrid beamforming design. Block diagonalization can be used to obtain hybrid precoder and combiner in a MU-MIMO system [116]. This paper mainly focuses on the analog RF processing design. High channel gain is obtained by analog beamforming using Fourier transform bases at the RF combiners and the phases of channel coefficients and combiners at the BS. The inter-user interference is canceled by the digital beamforming. A rank constraint resource allocation and hybrid beamforming technique using semidefinite programming have been proposed in [18]. The analog and digital precoders are obtained to maximize the proportional fair throughput in multiuser MIMO-OFDM system. Jiang et al. [165] propose another joint user scheduling and MU-MIMO hybrid beamforming scheme for OFDM mmWave system. It first allocates the frequency domain resources to the users' set with optimal beam, and then the analog beamforming vectors apply the optimal beam of each MU-MIMO user and the digital beamforming is realized by weighted MMSE to mitigate the residual inter-user interference. A consolidated list of reported developments in hybrid beamforming with $1 B S N_{t}$ and $K U E N_{r}$ is presented in Table XII.

5) Hybrid Beamforming: $L N_{t}$-antenna $B S s$ and $K$ singleantenna UEs in each cell (LBSN $N_{t}$ and KLUE1): In a multicell multiuser MIMO system, inter-cell interference is a critical problem. Interference mitigation techniques rely on the inter-cell coordination which incurs overhead. This problem becomes more severe in massive MIMO systems which require large amount of feedback information for channel estimation. Due to the excessive feedback required for the channel estimation in massive MIMO systems, TDD transmission mode has been widely adopted for the design and analysis of the mmWave massive MIMO systems. It leverages the channel reciprocity for simultaneous channel estimation in the uplink and downlink. The uplink pilot contamination causes channel estimation errors at BS which in turns causes inter-cell interference in downlink. Within a cell, the pilot signals used by UEs are mutually orthogonal, however, the re-use factor of one among neighboring cells causes pilot contamination in the considered cell. In the presence of inter-cell and intra-cell interference the receive signal at the output of the BS combiner can be written as

$$
\begin{aligned}
\mathbf{Y} & =\mathbf{W}^{D B^{*}} \mathbf{W}^{A B^{*}} \mathbf{H s}+\mathbf{W}^{D B^{*}} \mathbf{W}^{A B^{*}} \mathbf{G} \mathbf{x} \\
& +\mathbf{W}^{D B^{*}} \mathbf{W}^{A B^{*}} \mathbf{n}
\end{aligned}
$$

where $\mathbf{H}$ and $\mathbf{G}$ represent the data-channel and interferencechannel matrices, respectively; $\mathbf{s}$ is the intended signal vector, $\mathbf{x}$ is the interference signal vector; $\mathbf{W}^{D B}, \mathbf{W}^{A B}$ are the digital and analog combiners at the BS, and $\mathbf{n} \sim \mathcal{C N}\left(0, \sigma^{2}\right)$ is the AWGN noise with zero mean and variance $\sigma^{2}$. The BS maximizes the selected objective over the optimization variables $\mathbf{W}^{D B}$ and $\mathbf{W}^{A B}$ such that $\left\|\mathbf{W}^{A B}\right\|^{2}=1$ and $\left\|\mathbf{W}^{A B^{*}} \mathbf{G}\right\|=0$. The first constraint ensures the uni-modulus nature of analog beamformer and the second constraint is used for interference 
TABLE XII: Hybrid Beamforming with $1 B S N_{t}$ and $K U E N_{r}$

\begin{tabular}{|c|c|c|c|c|c|}
\hline Paper & Solution Method & Analog BF matrix & Digital BF matrix & RF Chains & SE performance metric \\
\hline $\begin{array}{l}\text { Nguyen et al. } \\
{[163]}\end{array}$ & OMP & $\begin{array}{l}\text { Transmit array response } \\
\text { vectors assuming perfect } \\
\text { AoD knowledge }\end{array}$ & $\begin{array}{l}\text { For known } F^{A B} \text {, mini- } \\
\text { mize the sum-MSE }\end{array}$ & $K \leq N_{R F}$ & $\begin{array}{l}38 \mathrm{bps} / \mathrm{Hz} \text { at } 0 \mathrm{~dB} \text { with } \\
N t=64, N_{r}=16, K= \\
N_{R F}=8 .\end{array}$ \\
\hline Jing et al. [75] & $\begin{array}{l}\text { Compress sensing on } \\
\text { feedback CSI }\end{array}$ & $\begin{array}{l}\text { Conjugate transpose of } \\
\text { downlink channel }\end{array}$ & ZF & $K \leq N_{R F}$ & $\begin{array}{l}1.37 \mathrm{bps} / \mathrm{Hz} \text { at } 0 \mathrm{~dB} \text { with } \\
N_{t}=64, N_{r}=1, K= \\
N_{s}=2 .\end{array}$ \\
\hline Lin et al. [126] & $\begin{array}{l}\text { Beamspace and beam se- } \\
\text { lection }\end{array}$ & $\begin{array}{l}\text { Butler matrix and tree } \\
\text { search based beam selec- } \\
\text { tion }\end{array}$ & $\begin{array}{l}\text { MMSE to cancel interuser } \\
\text { interference }\end{array}$ & $N_{s}=K \leq N_{R F}$ & $\begin{array}{l}5 \mathrm{bps} / \mathrm{Hz} \text { at } 0 d B \text { with } \\
N_{t}=64, N_{R F}^{t x}=8, K= \\
4, N_{r}=4, N_{R F}^{r x}=1 .\end{array}$ \\
\hline $\begin{array}{l}\text { Alkhateeb et al. } \\
\text { [147] }\end{array}$ & Quantized codebook & Beamsteering codebook & $\begin{array}{l}\text { ZF on the effective chan- } \\
\text { nel }\end{array}$ & $N_{s}=K \leq N_{R F}$ & $\begin{array}{l}6.8 \mathrm{bps} / \mathrm{Hz} \text { at } 0 \mathrm{~dB} \text { with } \\
N_{t}=64, N_{R F}^{t x}=N_{s}= \\
K=4, N_{r}=16 .\end{array}$ \\
\hline $\begin{array}{l}\text { Garcia et al. } \\
{[164]}\end{array}$ & DFT implementation & $\begin{array}{l}\text { DFT-based } \\
\text { connected }\end{array} \begin{array}{l}\text { Fully- } \\
\text { beamforming network }\end{array}$ & ZF precoding & $K<N_{R F}<N_{t}$ & $\begin{array}{l}60 \mathrm{bps} / \mathrm{Hz} \text { at } 0 \mathrm{~dB} \text { with } \\
N_{t}=64, N_{R F}=32, \\
K=4, N_{r}=1 .\end{array}$ \\
\hline Ni et al. [116] & $\begin{array}{l}\text { Hybrid block diagonaliza- } \\
\text { tion }\end{array}$ & $\begin{array}{l}\text { Equal gain transmission } \\
\text { method [136] }\end{array}$ & $\begin{array}{l}\text { Block diagonalization of } \\
\text { effective channel }\end{array}$ & $\begin{array}{l}\text { BS: } K N_{S}<N_{R F}<N_{t}, \\
\text { UE: } N_{s}<N_{R F}<N_{r}\end{array}$ & $\begin{array}{l}80 \mathrm{bps} / \mathrm{Hz} \text { at } 0 d B \text { with } \\
N_{t}=256, N_{R F}^{t x}=16, \\
K=8, N_{r}=16, N_{R F}^{r x}= \\
2, N_{s}=2 .\end{array}$ \\
\hline Ahmed et al. [18] & $\begin{array}{l}\text { Discipline } \\
\text { programming }\end{array}$ & QR-decomposition & QR-decomposition & $N_{s}=K \leq N_{R F}$ & $\begin{array}{l}2.5 b p s / H z \text { at } 0 d B \text { with } \\
N_{t}=64, N_{R F}=16, \\
K=8, N r=1 .\end{array}$ \\
\hline Jiang et al. [165] & $\begin{array}{l}\text { Joint radio resource allo- } \\
\text { cation and hybrid beam- } \\
\text { forming }\end{array}$ & $\begin{array}{l}N_{R F} \text { column vectors } \\
\text { selection from quantized } \\
\text { codebook }\end{array}$ & Weighted MMSE & $N_{s}<=K \leq N_{R F}<=N_{t}$ & $\begin{array}{l}10.15 \mathrm{bps} / \mathrm{Hz} \text { at }-10 \mathrm{~dB} \\
\text { with } N_{t}=512, N_{R F}^{t x}= \\
2, K=2 .\end{array}$ \\
\hline
\end{tabular}

nulling.

In [166], authors propose a multi-layer hybrid precoding structure to combat the inter-cell and intra-cell interferences in a multi-cell system with $L$ cells/BSs and $K$ single antenna users in each cell. They form a three stage precoding matrix. Each matrix is designed to achieve only one precoding objective, i.e., maximizing desired signal power, minimizing inter-cell interference, and minimizing intra-cell interference. Zhu et al. [167] present a hybrid beamforming framework and the corresponding channel estimation techniques to tackle the pilot contamination. The hybrid beamforming framework consists of a high-dimension analog and a low-dimension digital beamformers. They develop a hybrid beamforming solution with the Kronecker decomposition of the analog beamforming and channel vectors into Kronecker products of phase-shift factors. In this framework, the inter-cell interference is canceled by analog beamformer and then the low-dimension digital beamformer demultiplexes the users' data streams by suppressing the intra-cell interference. A fully connected hybrid beamforming is compared with digital beamforming and analog beamforming in a dense urban environment [168]. It has been found that in a particular multi-cell scenario with $K$ multiantenna users and LOS environment, the hybrid beamforming with $N_{R F}=16$ gives the performance near to pure digital beamforming with $N_{t}=64$.

Most of the work on multi-cell multiuser massive MIMO systems focus on the TDD transmission mode to tackle the huge feedback required for channel estimation. But the current cellular systems commonly use FDD. In [145], authors consider a massive MIMO FDD system for pilot signal design along with the transmit hybrid precoder design. The proposed pilot sequence aims to minimize the average channel MSE using Kalman filtering framework. The proposed solution can be applicable on the sub $6 \mathrm{GHz}$ cellular networks which can be further seamlessly integrated with current deployed FDD cellular networks. The summary of hybrid beamforming with $L B S N_{t}$ and $K L \mathrm{UE} 1$ is shown in Table XIII.

6) Hybrid Beamforming: $K N_{t}$-antenna UEs and a BS with $N_{r}$ antennas system $\left(K U E N_{t}\right.$ and $\left.1 B S N_{r}\right)$ : In this type of system model, an uplink of a single cell where $K N_{t}$-antenna UE transmit to $N_{r}$-antenna BS is investigated. A multiuser uplink channel estimation scheme for mmWave massive MIMOOFDM systems is presented in [148]. It uses distributed compressive sensing and exploits the angle-domain structured sparsity of mmWave frequency selective fading channels for the reduced training overhead. The power leakage problem due to the continuous estimation of AoA/AoD is solved by the grid matching pursuit scheme. In mmWave multiusers uplink, BS receives the signals from multiple users with probably similar AoA, which results in severe inter-user interference. This interference can be minimized by the hybrid beamforming design at the BS where both digital and analog beamformers are designed to minimize the inter-user interference [170]. Li et al. [170] derive the analog beamformer based on the Gram-Shmidh method to reduce the inter-user interference and MMSE based digital beamformer is derived from the effective channel. A switch-based low complexity combiner for multiuser massive MIMO system has been proposed in [51]. The resultant non-coherent combining problem with quantized combining vectors is replaced by the quasi-coherent combining problem and solved by a low complexity greedy algorithm which is polynomial in terms of the number of antennas. Table XIV summarizes the methods used to obtain the analog and digital matrices for hybrid beamforming with $K \mathrm{UEN} N_{t}$ and $1 \mathrm{BS} N_{r}$.

7) Hybrid Beamforming: Backhaul system $\left(1 B S N_{t}\right.$ and $\left.1 B S N_{r}\right)$ : This configuration is a practical example of backhaul link. The exponential growth in mobile data requires high 
TABLE XIII: Hybrid Beamforming with $L B S N_{t}$ and $K L U E 1$

\begin{tabular}{|c|c|c|c|c|c|}
\hline Paper & Solution Method & Analog BF matrix & Digital BF matrix & RF Chains & SE performance metric \\
\hline $\begin{array}{l}\text { Alkhateeb et al. } \\
\text { [166] }\end{array}$ & Multi-layer precoding & 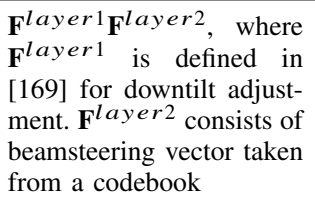 & $\begin{array}{l}F^{\text {layer } 3} \text { for canceling the } \\
\text { intra-cell multiuser inter- } \\
\text { ference. }\end{array}$ & $N_{R F}<N_{t}$ & $\begin{array}{l}24 \mathrm{bps} / \mathrm{Hz} \text { at } 10 \mathrm{~dB} \text { with } \\
N_{t}=120 \times 30 \text { planar } \\
\text { array, } K=20, L=7\end{array}$ \\
\hline Zhu et al. [167] & $\begin{array}{l}\text { Dividing the optimization } \\
\text { problem into sequential } \\
\text { problems of designing } \mathrm{DB} \\
\text { and } \mathrm{AB} \text { separately }\end{array}$ & $\begin{array}{l}\text { Kronecker product factor } \\
\text { of channel matrix }\end{array}$ & MMSE precoder & $K=N_{R F}<<N_{t}$ & $\begin{array}{l}34 \mathrm{bps} / \mathrm{Hz} \text { at } 0 d B \text { with } \\
N_{t}=128, N_{R F}^{t x}=4, \\
K=4, N_{R F}^{r x}=2 .\end{array}$ \\
\hline $\begin{array}{l}\text { Gimenez et al. } \\
{[168]}\end{array}$ & Monte-Carlo & $\begin{array}{l}\text { DFT matrix based code- } \\
\text { book }\end{array}$ & MRT precoder & $K<=N_{R F}<<N_{t}$ & Not available. \\
\hline Noh et al. [145] & $\begin{array}{l}\text { Kalman filter based } \\
\text { channel estimation and } \\
\text { Toeplitz distribution } \\
\text { theorem for hybrid } \\
\text { beamforming }\end{array}$ & $\begin{array}{l}\text { Toeplitz matrix decompo- } \\
\text { sition by DFT matrices }\end{array}$ & $\begin{array}{l}\text { Toeplitz matrix decompo- } \\
\text { sition by DFT matrices }\end{array}$ & $1<=N_{R F}<<N_{t}$ & $\begin{array}{l}6 b p s / H z \text { at } 0 d B \text { with } \\
N_{t}=32, K=5\end{array}$ \\
\hline
\end{tabular}

TABLE XIV: Hybrid Beamforming with $K \mathrm{UE} N_{t}$ and $1 \mathrm{BS} N_{r}$

\begin{tabular}{|c|c|c|c|c|c|}
\hline Paper & Solution Method & Analog BF matrix & Digital BF matrix & RF Chains & SE performance metric \\
\hline Gao et al. [148] & $\begin{array}{l}\text { Distributed grid matching } \\
\text { pursuit }\end{array}$ & $\begin{array}{l}\text { Iterative algorithm in an- } \\
\text { gular domain }\end{array}$ & $\begin{array}{l}\text { Iterative algorithm in an- } \\
\text { gular domain }\end{array}$ & $\begin{array}{l}\text { BS: } K=N_{R F}<<N_{r}, \\
\text { UE: } 1=N_{R F}<<N_{t}\end{array}$ & $\begin{array}{l}8.8 b p s / H z \text { at } 0 d B \text { with } \\
K=4, N_{t}=32, N_{R F}^{t x}= \\
1, N_{r}=128, N_{R F}^{r x}=4 .\end{array}$ \\
\hline Li et al. [170] & Reduce interuser interference & Gram-Schmidt method & MMSE beamforming & BS: $K=N_{R F}<N_{r}$ & $\begin{array}{l}50 \mathrm{bps} / \mathrm{Hz} \text { at } 0 d B \text { with } \\
K=2, N_{t}=1, N_{r}= \\
128, N_{R F}^{r x}=8 .\end{array}$ \\
\hline $\begin{array}{l}\text { Alkhateeb et al. } \\
\text { [51] }\end{array}$ & Switch-based combining & Quasi-coherent combining & 1 & $K \leq N_{R F}<N_{r}$ & $\begin{array}{l}2.8 b p s / H z \text { at } 0 d B \text { with } \\
K=3, N_{t}=1, N_{r}=64, \\
N_{R F}^{r x}=3 .\end{array}$ \\
\hline
\end{tabular}

capacity backhaul links in next generation HetNets. mmWave massive MIMO backhaul links with hybrid beamforming help solve the backhaul problems. The hybrid beamforming offers an optimal performance and complexity trade-off. Multiple RF chains allow data-stream multiplexing between the TX and the RX, which can be used in backhaul to enhance the overall throughput. The hybrid beamforming is also applicable in point-to-multipoint scenarios, enabling the TX to send multiple streams to independent RXs simultaneously. This may further improves the backhaul network capacity [171]. The ultra-dense network has been considered as a promising candidate for future 5G cellular networks. These networks are composed of a macro BS and a number of small-BSs. Usually, the macro BS is connected with small-BSs through optical fiber. However, mmWave massive MIMO has paved a way to cost effective and readily installable solution. The mmWave can provide the Giga-bit/sec traffic for backhauls. In [149], a digitally controlled phase shifter network based hybrid beamforming and the associated compressive sensing based channel estimation is proposed.

8) Hybrid Beamforming: Multiple UEs interference model $\left(K U E N_{t}\right.$ and $\left.K U E N_{r}\right)$ : It is a general multiuser massive MIMO interference model, which is used to design and analyze the hybrid beamforming solutions. MMSE based Hybrid beamforming design for mmWave massive MIMO interference system is presented in [172]. Sparse approximation problems are formulated and solved by orthogonal matching pursuit based algorithms to select the near-optimal analog beamformer and optimize the corresponding digital beamformer.

\section{B. Relation with the standardization activities}

MmWave band, particularly at $60 \mathrm{GHz}$, has received significant attention in the last decade from the WLAN and WPAN community. Specifically, two IEEE standards of mmWave have been proposed for IEEE 802.11ad and IEEE802.15.3c. Although the hybrid beamforming has not been utilized in the current standardization of IEEE 802.11ad and IEEE 802.15.3.c, the mentioned related works in Section IV have validated that the hybrid beamforming is a potential candidate for achieving low-complexity mutli-user BF in mmWave communication systems. For example, it has been proved that hybrid beamforming can achieve close to the optimal digital beamforming performance with reduced complexity [173]. In addition, an overhead involved in the incorporation of hybrid beamforming in IEEE 802.11ad has been studied in [174]. The simulation results demonstrate that the beamforming performance increases and then decreases with the increase of a number of users. Therefore, it is suggested that the hybrid beamforming architectures can support only a finite number of users. In addition, there are many open questions related to the architecture and signal processing level of the hybrid beamforming and are listed in the future research directions. These future research directions can not only be helpful in the standardization of the hybrid beamforming in IEEE $802.11 \mathrm{ad}$ and IEEE $802.15 .3 \mathrm{c}$ but also in the future $5 \mathrm{G}$ wireless networks. 


\section{Future Research Directions}

In case of $1 \mathrm{BS} N_{t}$ and 1UE1 system, designing codebooks for different kind of antenna arrays would be an interesting extension in [140]. The single-user hybrid beamforming with mobility in [141] could be extended for multiuser scenario, towards optimizing the number of RF chains allocated for each user and designing the multiuser hybrid beamforming.

In point-to-point downlink (1BSN $N_{t}$ and $1 \mathrm{UE} N_{r}$ ), the proposed heuristic approach in [84] can be extended to MUMIMO and more practical scenario of imperfect CSI. The decoupled approach to mmWave transceiver design [42] can be used for direct joint optimization of hybrid beamforming. Moreover, the problems in [42, eq. (17)] and [42, eq. (24)] can be solved by algorithms for simultaneously sparse approximation [175]. It will be interesting to extend the alternating minimization techniques in [107] to switch-based hybrid precoder design problems. Another extension is to consider the hybrid precoder design combined with channel training and feedback. A detailed convergence analysis and optimality characterization of the proposed algorithms could be a significant contribution. The hybrid beamforming design [106] could be evaluated for the trade-off between the achieved SE and the consumed energy of the dynamic subarray structure, and could be compared with the fullyconnected and the fixed-subarray architectures. The wideband mmWave hybrid beamforming design with limited feedback [80] could be extended to exploit the frequency correlation of mmWave channels to further reduce the feedback overhead. Also, another important extension would be to design efficient hybrid precoding codebooks for wideband multiuser mmWave systems. The running-time complexity of beamforming algorithms in [156] could be investigated using block diagonalization method and considered non-uniform linear arrays using genetic algorithm under imperfect channel estimation. It would be interesting to consider the mmWave channel estimation work [117] with random blockage between the BS and UE and design the adaptive channel estimation algorithms. It would be also important to develop efficient algorithms that adaptively estimate the channel with random or time-varying array manifolds. A possible extension of [141] work could be optimizing the number of RF chains allocated for each user in multiuser massive MIMO system. [118] could be modified to work with a UPA in terms of unitary structures, because array manifold of UPA is a Kronecker product of ULA manifolds.

In point-to-multipoint massive MIMO system $\left(1 \mathrm{BS} N_{t}\right.$ and $K$ UE1) [49], the user scheduling and sub-carrier allocation algorithm may not necessarily converge to the global optimal solution, and the development of global optimal user scheduling and sub-carrier allocation algorithm for the presented hybrid beamforming design is not trivial, and it is still an open research problem. Another interested extension could be the comparison of the given beamforming structures for cost and energy consumption. The generalization of the wideband MU-MIMO hybrid beamforming [161] to the case that the analog beamforming is realized by a partially connected phase shifter network, and in mmWave frequencies is a possible research work. The MMSE based hybrid channel estimator for imperfect CSI in [124] can be used to investigate the trainingthroughput trade-off for imperfect channel covariance matrix between BS and UE. It would be interesting to replace the uncorrelated Rayleigh fading channel in [93] by a realistic mmWave geometric channel to evaluate the performance of MU-MIMO system.

For a downlink of a single cell multiuser massive MIMO system $\left(1 \mathrm{BSN} N_{t}\right.$ and $\left.K \mathrm{UE} N_{r}\right)$, low complexity beamspace MIMO and beam selection based hybrid beamforming in [126] can be extended with adaptive power allocation and imperfect CSI. It would be interesting to investigate the EE of the hybrid beamforming based on S-parameters in [164] with mmWave geometric channel model. As a future work, it is of interest to extend the [147] to develop efficient mmWave precoding and channel estimation algorithms for multiuser cellular systems taking into consideration the inter-cell interference.

As a future work in a multi-cell multiuser MIMO system $\left(L B S N_{t}\right.$ and $\left.K L \mathrm{UE} 1\right)$, it would be interesting to investigate and optimize the multi-layer precoding using different hybrid beamforming structures in [166]. It is also of interest to develop techniques for the channel training and estimation under impaired hardware constraints. The design of the hybrid beamforming while considering pilot contaminated channel estimation and hardware impairment [167] could be extended for inter-cell interference cancellation in broadband channel in analog domain. Another interesting application would be the application of Kronecker analog beamformer on other antenna configurations, e.g., circular or cylindrical arrays. It is of interest to investigate the performance of hybrid beamforming case study in [168] when different planar array solutions and codebook designs are used with imperfect CSI. Reference [145] considers flat Rayleigh-fading channel which has limited applications in mmWave massive MIMO systems. A more practical geometrical channel model, like, extended SalehValenzuela model [42] could be used to design the hybrid beamforming framework.

In a multiuser massive MIMO uplink ( $K \mathrm{UE} N_{t}$ and $\left.1 \mathrm{BS} N_{r}\right)$, it would be interesting to evaluate the performance of the switch-based beamforming architecture [51] by considering the impact of practical factors like switching speed, switches imperfect isolation, and insertion losses.

\section{Hybrid BeAmforming: Resource Management}

MmWave massive MIMO systems leverage advantages and disadvantages. An intelligent and smart design of MAC can benefit from the value added features like small form factor for antenna arrays, pencil beams, and compensate the weaknesses like large path loss, rain absorption etc.

\section{A. Resource Block Allocation}

The mmWave massive MIMO system defines the resource block (RB) in the time, frequency, and space domain in contrast to long term evolution (LTE), which uses timefrequency RB. In order to optimally use the three dimensional time-frequency-space RB, one needs complete CSI knowledge both at the TX and the RX, which is not feasible 
in mmWave massive MIMO systems due to the high complexity of pilot transmission and channel estimation. Hybrid beamforming makes the massive MIMO channel estimation feasible by employing the low dimensional baseband digital beamformer along with high dimensional RF analog beamformer. In mmWave systems, the closely located UEs can be treated as a group and BS can use separate analog beam for each group [176]. In mmWave HetNets' backhauls, this grouping technique can be applied on a group of adjacent small BSs [177]. Analog beamforming can control the spatial part of time-frequency-space RB and the digital beamforming realizes the multiplexing within a group. This is particularly important for the case of an ultra-dense environment, in which beamforming can be used for spatial allocation of resources and thus, can improve the system capacity by reducing the interference. Therefore, it is evident that a combination of low-dimensional analog beamforming and high dimensional beamforming can be used for optimizing time-frequency-space resources.

\section{B. Beam Management}

Beamforming helps in the addition of space domain which allows concurrent frequency domain RB allocation to different UEs in the same time slot. The mmWave massive MIMO based future mobile networks will consist of virtual cells. A virtual cell is defined as a set of UEs served by the same analog beamformer of the BS but not necessarily located closely in an area centered by the BS. The fixed area size cell boundaries of traditional cellular would no longer exist in the future mmWave massive MIMO systems. Narrow beams can serve distant UEs without interfering other UEs provided that there is no obstacle between BS and intended UE. On the other hand, a closely located UE may deprive of connection due to the obstacles. The hybrid precoding and combining offer extra degree of freedom in space domain with large number of antennas and analog beamforming. The virtual cell could dynamically change depending upon the UE traffic load, the channel between BS and UE, and the BS load [178].

Generally, two types of beam management are common in mmWave multiuser massive MIMO systems: 1) hybrid beamforming for single-antenna grouped UEs and 2) hybrid beamforming for multiple-antenna UEs.

Hybrid beamforming for single-antenna grouped UEs: In a group based hybrid beamforming, the BS with $N_{t}$ transmit antennas and $N_{R F} \mathrm{RF}$ chains (such that $N_{R F}<N_{t}$ ) can group UEs together based on the average CSI and direct a single beam towards a group of UEs that have similar covariance matrix using one beamforming vector of the analog beamforming matrix [179]. In a similar way, the beam allocation to different groups is carried out under time-frequency-space scheduling in MAC layer. In order to get maximum benefits from hybrid beamforming, the following things need attention such as a careful scheduling design of time, bandwidth, and the area by considering the coherence time, coherence bandwidth, and the covariance matrix of the channel.

Hybrid beamforming for multiple-antenna UEs: In case of multiple antennas at UE, UEs are also capable of beamforming. The multi-beam capability at UE entails simultaneous connections with multiple MBSs or small BSs [180]. It significantly improves the received signal quality and coverage. In addition, the stringent $5 \mathrm{G}$ network requirements can be met by exploiting smart antenna technologies which can provide spatial degree of freedom. However, the only limiting factor here is the number of required RF chains, which can also be reduced by using optimized hardware architectures.

Since the $5 \mathrm{G}$ network is expected to be extremely dense and heterogeneous, it becomes challenging to meet such stringent $5 \mathrm{G}$ requirements. Therefore, the beam management, in either of its two mentioned forms, can potentially help in acquiring the benefits such as SNR improvement, interference avoidance and rejection, and network efficiency and can lead to the performance improvement of hybrid beamforming in $5 \mathrm{G}$ network.

\section{Medium access management}

In this section, we address, why the conventional MAC design methods are not suitable for mmWave systems and what should be taken into account while proposing MAC designs for mmWave systems.

In mmWave systems, it is important to use directional beams for mitigating the effect of higher path losses at mmWave frequencies. In an outdoor environment, the standard MAC methods, such as CSMA, which is used for interference management by listening to neighbor nodes are not suitable here. Because the directivity of directional beams makes interference among neighboring transmission links almost negligible [181]. Therefore, the conventional MAC designs are not applicable for mmWave system. On the other hand, in an indoor environment, the negligible interference due to the high directionality of the beams is no longer valid. In both the cases, deafness is considered as one of the most critical problem, which arises due to directional communication in beamforming antennas. Therefore, the MAC design of mmWave system should address the deafness problem. One possible way to address the deafness problem is to have a coordination among BSs.

The limited range of mmWave links require a large number of APs, which further introduces extra interference components such as intra-APs and inter-AP. In order to address these problems there has been some work related to directional MAC for mmWave systems [182], [183] and [184] and all the protocols are based on TDMA, as CSMA/CA is difficult to visualize for mmWave systems. However, a TDMA-based MAC protocol can cause either under- or over-allocated time for the users. Although several standards defined for MAC design of mmWave system are based on TDMA, there is a need for further exploration due to an unfair utilization of the time slots in TDMA. There are also some efforts for designing MAC for mmWave system based upon a centralized architecture. A directive CSMA/CA protocol is proposed in [185] in which virtual carrier sensing is utilized for behaving like CSMA/CA; however, it does not fully exploit the spatial resource for enhancing the system capacity. The authors in [186] propose an adaptive multicast group in which the beam widths are generated based on the location of the multicast 
devices. For the outdoor mesh network for mmWave system, there has been some efforts for distributed MAC protocol designing [187]. In [187], a memory-guided distributed MAC protocol is proposed in which a Markov state transition diagram is included for avoiding a deafness problem. It employs memory for scheduling the slots and it does not fully exploits the spatial reuse. Although some work has been done on directional MAC for mmWave system, most of the solutions do not take into account the deafness problem which is a real challenge in the MAC design of mmWave systems.

\section{Initial Search and Tracking}

In this section, we present initial search and tracking of beams for mmWave system which is of vital importance in hybrid beamforming. This is because if the cellular BS have to use mmWave technologies, then the directional antennas in the BS should be able to track the users; otherwise, supporting mobility is no longer possible. We summarized the initial search and tracking possibilities in four ways. First, an exhaustive search beamforming can be done via two ways: transmit beamforming and receive beamforming [188]. In the transmit beamforming, there are training indicators (TI) and beam-transponders (BT). Initially, the TI sweeps through beams by transmitting one packet in each direction and the $\mathrm{BT}$ receives the packet within an omni-directional antenna pattern. Based on the received SINR, TI and BT exchange their roles and together learn their own optimal directions. In the RX beamforming, TI transmits in omni-direction, BT scans the direction, and finally they come up with optimal directions. Second, in IEEE, there are two standards of mmWave communication such as IEEE 802.15.3c WPAN [189] and IEEE 802.11ad WLAN [190]. In both the standards, a two-stage beam-forming and training operation is utilized: coarse-grained beam training and fine-grained beam training. Although the coarse-grained and fine-grained protocol is faster than the exhaustive search one, still it is slow as pointed out in [190]. Third, the details of the interactive beam training is presented in [190]. The main philosophy behind it is that it searches the optimal direction via the exhaustive training and once it finds the reasonable optimal configuration then it stops with the concern of reducing the complexity. Fourth, in order to fasten the average speed, the order of beams can be prioritized. Although some propositions for initial searching and tracking are listed above, it is also important to explore further on the complexity, accuracy, and timing related issues in searching and tracking.

\section{HyBRID BEAMFORMING IN HETNETS}

HetNets are considered as one of the key building blocks of $5 \mathrm{G}$ wireless networks, which guarantee capacity and coverage enhancement. The overall capacity of HetNets can be enhanced by employing hybrid beamforming. Based on these lines, in this section, we list the key efforts that have been done in hybrid beamforming for HetNets and particularly, what are the future challenges that can further improve the performance of HetNets.
Primarily, HetNet is classified as a RAN which encompass traditional macro cells and many small cells, where the small cells (including femto cells, pico cells and micro cells) are categorized by the range they provide. Traditionally, SBSs are connected to main BSs through optical fiber. In 5G wireless networks, it is anticipated that small cells will provide an ultra-dense network and coverage for meeting the stringent $5 \mathrm{G}$ capacity requirements. However, due to ultra-dense small cell network in $5 \mathrm{G}$ wireless networks, it is impossible to realize such optical fiber deployment everywhere. Fortunately, mmWave massive MIMO is considered as a candidate to realize backhaul networks, since it is cost effective and a readily installable solution which can provide a giga-bits/sec connection. Considering the importance of mmWave massive MIMO and hybrid beamforming in HetNets, we categorize the related work into three classes BS/UE configuration, signal processing, and implementation and are also given in Table $\mathrm{XV}$.

The authors in [191] focus on the combination of massive MIMO and dense small cells. Both massive MIMO and dense small cells are used for spatial reuse of the spectrum, where the former support spatial multiplexing of more users, while the later support of more users by densification. Although there are some works which consider both as extremes cases (or competitors) [196], in fact it is not true and they can be combined as [197], [198]. The authors investigated clustered small cells deployments with two dimensional active antenna array and the performance in terms of throughput is measured by varying the number of antennas and small cell density. Simulation results validate the superior performance of the joint MIMO and dense small cells compared to the benchmarks. Thus, it is evident that the stringent $5 \mathrm{G}$ requirements can be satisfied by combining the benefits of both massive MIMO and dense small cells. However, in order to reduce the complexity incurred by massive MIMO, hybrid beamforming can provide an adequate trade-off between performance and complexity. The authors in [165] present a joint user scheduling and multi user hybrid beamforming for the downlink of MIMO OFDMA systems. The proposed system comprises of two steps. First, the user scheduling is carried out and the users having a similar optical beam form an OFDMA user group. Second, the BS allocates the frequency resources to each formed OFDMA group, which is considered as a virtual user to the BS. Moreover, the best beam for each user in MIMO is selected by analog beamforming and then the digital beamforming algorithm is solved using MMSE for achieving the best performance gain and for mitigating the user interference.

The authors in [192] present a user association problem in MIMO and mmWave enabled HetNet environments. They considered a heterogeneous environment where macro cells utilize MIMO while small cells utilize mmWave. Further, it is also assumed that the BSs are solely operated on renewable energy. The problem is formulated in a manner that the network utility is maximized without passing the threshold on the harvested energy on the BSs. The investigation concluded that increasing the number of antennas at the macro BS increases the throughput but at the cost of more power consumption at the BS. On the other hand, the introduction of mmWave 
TABLE XV: Hybrid Beamforming in HetNets

\begin{tabular}{lccc}
\hline \multicolumn{1}{c}{ Paper } & BS/UE configurations & Signal processing & Implementation \\
\hline Hao et al. $[191]$ & $\checkmark$ & & \\
Jiang et al. $[165]$ & $\checkmark$ & & \\
Xu et al. $[192]$ & $\checkmark$ & $\checkmark$ & \\
Gao et al. $[149]$ & & $\checkmark$ & \\
Wu et al. $[193]$ & & & $\checkmark$ \\
Pi et al. $[194]$ & & & $\checkmark$ \\
Hur et al. al $[195]$ & & & \\
\hline
\end{tabular}

BSs or antennas enhances the throughput further. Finally, they demonstrate that mmWave based small cells play a key role in enhancing the overall throughout of the network. Although a variety of $\mathrm{BS} / \mathrm{UE}$ configurations are listed above, still it is an open question as what is the most optimized configuration considering the improved performance, scalability, reduced information exchange overhead, etc.

The authors in [149] propose a digitally controlled phase shifter network-based hybrid precoding and combining scheme for mmWave MIMO for ultra-dense networks. Specifically, the low-rank property of mmWave MIMO channel matrix allows implementation of low cost and less-complex transceiver architecture with negligible performance overhead. The important concern in this regard is that the macro BS is able to receive multiple streams from the small cells, which is not the case with conventional hybrid precoding and combining schemes. The scheme also provides a viable solution for point to multipoint links for realizing mmWave in wireless backhaul. The authors in [193] propose a reconfigurable mmWave based hybrid beamforming system, which exploits the channel and antenna array diversities. Within their work, sub antenna array is configured for adjusting the spatial and polarization diversities and this configuration is done by considering the environment and the relative location between the TX and RX. In the proposed reconfigurable hybrid beamforming architecture, a training sequence mechanism is introduced both for TX and RX so that they can learn the best operation modes based on the current channel characteristics. Simulation results illustrate a significant improvement in $\mathrm{dBs}$ as compared to the normal hybrid beamforming system. The reconfigurable hybrid beamforming can potentially improve the performance of partially connected structure by adjusting the number of sub-arrays connected to analog front end and RF chain. Based upon the extended benefits of partially-connected architecture by reconfigurability, this architecture will assist in the standardization of hybrid beamforming for 5G networks.

The authors in [194] propose an optimized dynamic beamforming approach for utilizing mmWave as a backhaul for the $5 \mathrm{G}$ wireless networks. Specifically, they discuss potential array architectures and propose a mmWave wave gigabit broadband which provides an attractive solution for the fixed last-mile access and small cell backhaul for the 5G networks. Since small cells will be densely deployed in 5G network, it is extremely important to have a scalable network solution for small cell backhaul for the success of 5G. The dynamic beamforming approach by utilizing mmWave will provide a scalable backhaul solution which can also helps in improving the capacity at the last mile of 5G network. The authors in [195] propose an outdoor mmWave for the backhaul networking for $5 \mathrm{G}$ network. In order to overcome the problem of outdoor impairment, they investigated large array beamforming. However, such an environment requires the use of narrow beams, which are increasingly sensitive to various environmental concerns. The authors propose the use of adaptive sub space sampling and hierarchical codebooks for overcoming the sensitivity of the narrow beam, which can help in the standardization of access and backhaul in small cell networks by exploiting beamforming of mmWave.

\section{Future Research Directions}

Although the literature mentioned above discussed various solutions for the exploitation of hybrid beamforming in HetNets, the following topics have not been adequately addressed.

1) Dual band small cells: Dual access small cells operating on both licensed and unlicensed bands, respectively, are an integral part of $5 \mathrm{G}$. However, it is not yet clear how this integration steer the licensed band traffic to unlicensed band with creating harmful impact on the legacy unlicensed devices. As such it is interesting to investigate, how this integration affects the hybrid beamforming.

2) Optimization of antenna selection: The optimization of RF chains and the throughput of hybrid beamforming system in the presence of CSI errors and wideband mmWave needs further investigation [117], [199]. Apart from the advantages of the hybrid beamforming, there is still a need to investigate for the optimal selection of antenna arrays.

3) Handover and mobility management: Within the context of hybrid beamforming in mmWave systems, handover and mobility management are important concerns that need significant attention. In the dense deployment of small cells using mmWave, frequent handovers can occur even for the fixed users. This potential drawback of mmWave systems due to the presence of obstacles in the path, which is not the case in LTE environment. On one hand, frequency handover among the small cells can occur if the decision on the handover is based on RSSI-based mechanism, while on the other hand, loss of precise beamforming information can also happen. One possible way to remedy this problem is to support multiple connections for users so that in case of movement a transparent handover can be realizable.

4) Scheduling: The use of time-frequency-space separation using narrow beams helps in supporting a large number of concurrent transmission and correspondingly increasing the area 
SE, measured in bits $/ \mathrm{s} / \mathrm{Hz} / \mathrm{m}^{2}$. Traditionally, the scheduling mechanism of the resources is done in an omnidirectional environment, which leads to orthogonal time-frequency resources inside the cell. Considering the large number of antennas with limited RF chains, the BS can group a number of users as group based on the statics of the channels and serve each group with a co-variance channel matrix with different analog beam formers for optimizing the performance. Thus, it is interesting to investigate how the scheduling of resources, specifically for dense small cell environment utilizing mmWave, can be optimized for a hybrid beamforming environment.

5) Interference management: Generally, in small cellular environments there are three types of interference inter-cell, intra-cell and inter-layer interference. Inter-cell interference is the interference among the different cells belong to a similar tier. Intra-cell interference is the interference within the cell and inter-layer interference is the interference in different cells belong to different tiers. The traditional approaches for interference management for omni-directional cases are not applicable for directional beamforming environment. Hybrid beamforming design has a direct impact of the interference classes but until now this impact has not studies yet adequately been investigated.

\section{CONCLUSION}

This paper provides a comprehensive review of hybrid beamforming which is an integral part of the mmWave massive MIMO systems. While the two previous surveys on beamforming [23], [22] focused more inclined on mmWave channel characteristics and indoor use such as IEEE 802.11ad, $802.15 .3 \mathrm{c}$ or hybrid beamforming structures on the basis of the CSI types (instantaneous or average), this paper focuses on the implementation, signal processing, and application aspects of the hybrid beamforming. Specifically, this paper categorizes the hybrid beamforming into i) hybrid beamforming hardware architectures; ii) resource management; iii) different number of antennas at TXs and RXs, and the resulting digital and analog beamforming matrices; and iv) the hybrid beamforming in small cells and HetNets. For each of these topics, we gave an overview of the current state of the art research and also discussed the challenges, open issues, and possible future directions of research in the hybrid beamforming in mmWave massive MIMO systems.

\section{A. Lessons learned}

- Lesson 1: In summary, the hybrid beamforming spans hardware architecture, signal processing, resource management, and application areas. The ever-increasing demand of mobile data requires paradigm shift towards larger bandwidth available in mmWave frequency band, larger number of antennas and hence, the hybrid beamforming to save the capital and operation cost. The various hybrid beamforming architectures are applications specific, e.g., the fully-connected can achieve full beamforming gain per RF chain at the expense of hardware complexity, whereas, the low complexity sub-connected architecture provides low beamforming gain. From the power consumption point of view, bit resolution of ADC in $\mathrm{DB}$ and $\mathrm{PS}$ in $\mathrm{AB}$ plays the key role.

- Lesson 2: The mmWave massive MIMO system is only feasible with hybrid beamforming having low-dimension digital beamformer and low-dimension analog beamformer. The analog beamforming potential candidates are phase-shifters, switches, and antenna lens [22], whereas, the digital beamforming can utilize the established techniques on the effective channel to facilitate the multiple data streams. The aforementioned review of signal processing techniques for hybrid beamforming from system model perspective shows that there are relatively few works on the joint uplink pilot precoding and downlink channel estimation for optimal hybrid beamforming design. The inherent small cell size (100-200m) at mmWave frequencies requires multi-cell inter-cell and multiuser intra-cell interference management while designing the analog and digital beamformers.

- Lesson 3: Although resource management is not directly related to the hybrid beamforming, particularly at the architectural level, there are many resource management aspects that can have a significant impact on the performance of the hybrid beamforming. Specifically, the resource management aspects including user grouping, beam management, MAC variations both for indoor and outdoor, and initial searching and tracking are the potential research topics that need further exploration so that the overall performance of the hybrid beamforming can further be enhanced.

- Lesson 4: It is expected that the ultra-dense HetNet will be an integral part of the $5 \mathrm{G}$ wireless networks. Due to this, the challenges in hybrid beamforming intensify due to the densification of HetNet in the $5 \mathrm{G}$ network. Various hybrid beamforming aspects at the architectural level, signal processing, and resource management, both at the access and backhaul, become much more critical when the densification of HetNet is considered. In addition, the mmWave band is a potential candidate for the $5 \mathrm{G}$ backhaul part and many challenges need investigations such as dual-access small cells, optimization of an antenna array, time-frequency-space scheduling and interference management.

\section{REFERENCES}

[1] WWRF, "5G Vision, Enablers and Challenges for the Wireless Future," no. 16,2015

[2] Huawei, "5G : A Technology Vision," Huawei, White Pap., pp. 1-16, 2014.

[3] E. Hossain, M. Rasti, H. Tabassum, and A. Abdelnasser, "Evolution toward 5G multi-tier cellular wireless networks: An interference management perspective," IEEE Wirel. Commun., vol. 21, no. 3, pp. 118-127, jun 2014. [Online]. Available: http://ieeexplore.ieee.org/ stamp/stamp.jsp?tp $=\{\backslash \&\}$ arnumber $=6845056$

[4] W. Haerick and M. Gupta, "5G and the Factories of the Future," White Pap., pp. 1-31, 2015. [Online]. Available: https://5g-ppp.eu/wp-content/uploads/2014/02/ 5G-PPP-White-Paper-on-Factories-of-the-Future-Vertical-Sector.pdf

[5] L. Ericsson, "More than 50 billion connected devices," White Paper, 2011.

[6] "Incorporated," The 1000x data Challenge, 2013 
[7] Y. Chen, S. Zhang, S. Xu, H. T. Co, and W. H. Y. G. R. E. Volution, "Fundamental Trade-offs on Green Wireless Networks," IEEE Commun. Mag., vol. 49, no. 6, pp. 30-37, 2011.

[8] J. G. Andrews, S. Buzzi, W. Choi, S. V. Hanly, A. Lozano, A. C. Soong, and J. C. Zhang, "What will $5 \mathrm{~g}$ be?" IEEE Journal on selected areas in communications, vol. 32, no. 6, pp. 1065-1082, 2014.

[9] B. Bangerter, S. Talwar, R. Arefi, and K. Stewart, "Networks and devices for the $5 \mathrm{~g}$ era," IEEE Communications Magazine, vol. 52, no. 2, pp. 90-96, 2014

[10] A. F. Molisch, V. V. Ratnam, S. Han, Z. Li, S. L. H. Nguyen, L. Li, and K. Haneda, "Hybrid beamforming for massive mimo: A survey," IEEE Communications Magazine, vol. 55, no. 9, pp. 134-141, 2017.

[11] E. G. Larsson, O. Edfors, F. Tufvesson, and T. L. Marzetta, "Massive mimo for next generation wireless systems," IEEE communications magazine, vol. 52, no. 2, pp. 186-195, 2014.

[12] R. W. H. Jr, N. González-prelcic, S. Rangan, W. Roh, and A. M. Sayeed, "An overview of signal processing techniques for millimeter wave MIMO systems," IEEE J. Sel. Top. Signal Process., vol. 10, no. 3, pp. 436-453, apr 2016.

[13] A. L. Swindlehurst, E. Ayanoglu, P. Heydari, and F. Capolino, "Millimeter-wave massive MIMO: the next wireless revolution?" IEEE Commun. Mag., vol. 52, no. 9, pp. 56-62, sep 2014.

[14] S. Biswas, S. Vuppala, and J. Xue, "On the performance of relay aided millimeter wave networks," IEEE J. Sel. Top. Signal Process., vol. 10, no. 3 , pp. $576-588$, apr 2016.

[15] E. G. Larsson, O. Edfors, F. Tufvesson, and T. L. Marzetta, "Massive MIMO for next generation wireless systems," IEEE Commun. Mag. vol. 52, no. 2, pp. 186-195, feb 2014.

[16] L. Lu, S. Member, G. Y. Li, and A. L. Swindlehurst, "An overview of massive MIMO : Benefits and challenges," IEEE J. Sel. Top. Signal Process., vol. 8, no. 5, pp. 742-758, oct 2014.

[17] F. Rusek, D. Persson, B. K. Lau, E. G. Larsson, T. L. Marzetta, O. Edfors, and F. Tufvesson, "Scaling up MIMO: Opportunities and challenges with very large arrays," IEEE Signal Process. Mag., vol. 30, no. 1, pp. 40-60, jan 2013.

[18] I. Ahmed, H. Khammari, and A. Shahid, "Resource allocation for transmit hybrid beamforming in decoupled millimeter wave multiuserMIMO downlink," IEEE Access, vol. 5, no. 1, pp. 170-182, 2017.

[19] F. Boccardi, R. Heath, A. Lozano, T. L. Marzetta, and P. Popovski, "Five disruptive technology directions for 5G," IEEE Commun. Mag., vol. 52, no. 2, pp. 74-80, 2014.

[20] B. D. Van Veen and K. M. Buckley, "Beamforming: A versatile approach to spatial filtering," IEEE assp magazine, vol. 5, no. 2, pp. 4-24, 1988.

[21] EE359, "MIMO Systems : Channel Capacity, Beamforming , Diversity-Multiplexing Tradeoff , and RX Design," in Wirel. Commun. Lect. Notes, 2016. [Online]. Available: https://web.stanford.edu/class/ ee359/pdfs/lecture15.pdf

[22] A. F. Molisch, V. V. Ratnam, S. Han, Z. Li, S. L. H. Nguyen, L. Li, and K. Haneda, "Hybrid Beamforming for Massive MIMO - A Survey," arXiv Prepr. arXiv1609.05078, pp. 1-14, sep 2016. [Online]. Available: http://arxiv.org/abs/1609.05078

[23] S. Kutty, S. Member, and D. Sen, "Beamforming for millimeter wave communications : An inclusive survey," IEEE Commun. Surv. Tutorials, vol. 18, no. 2, pp. 949-973, 2016.

[24] S. Han, I. Chih-Lin, Z. Xu, and C. Rowell, "Large-scale antenna systems with hybrid analog and digital beamforming for millimeter wave 5g," IEEE Communications Magazine, vol. 53, no. 1, pp. 186194, 2015.

[25] V. Venkateswaran and A.-J. van der Veen, "Analog beamforming in mimo communications with phase shift networks and online channel estimation," IEEE Transactions on Signal Processing, vol. 58, no. 8, pp. 4131-4143, 2010.

[26] S. Han, C.-L. I, Z. Xu, and C. Rowell, "Large-scale antenna systems with hybrid analog and digital beamforming for millimeter wave 5G," IEEE Commun. Mag., vol. 53, no. 1, pp. 186-194, jan 2015.

[27] Nokia, "The 5G mmWave revolution," Nokia White Paper, 2017.

[28] H. Kallin, "Live 5G at Mobile World Congress 2017 I Ericsson Research Blog." [Online]. Available: https://www.ericsson.com/ research-blog/live-5g-mobile-world-congress-2017/

[29] Cisco, "Cisco Visual Networking Index: Global Mobile Data Traffic Forecast Update, 2016âĂŞ2021 White Paper - Cisco," 2017. [Online]. Available: https://www.cisco.com/c/en/us/solutions/collateral/service-provider/ visual-networking-index-vni/mobile-white-paper-c11-520862.html
[30] A. Biral, M. Centenaro, A. Zanella, L. Vangelista, and M. Zorzi, "The challenges of M2M massive access in wireless cellular networks," Digital Communications and Networks, vol. 1, no. 1, pp. 1-19, 2015.

[31] R. W. H. Jr, "Millimeter Wave MIMO Communication Heath Group in the WNCG @ UT Austin,” Tech. Rep., 2015.

[32] WirelessHD, "The WirelessHD Consortium serves to organize an industry-led standardization effort to define a next-generation wireless digital interface specification for consumer electronics and PC products." [Online]. Available: http://www.wirelesshd.org/

[33] WiGig, "Wi-Fi CERTIFIED WiGig । Wi-Fi Alliance." [Online]. Available: https://www.wi-fi.org/discover-wi-fi/wi-fi-certified-wigig

[34] H. Ji, Y. Kim, J. Lee, E. Onggosanusi, Y. Nam, J. Zhang, B. Lee, and B. Shim, "Overview of Full-Dimension MIMO in LTE-Advanced Pro," IEEE Communications Magazine, vol. 55, no. 2, pp. 176-184, 2017.

[35] 3GPP, "Release 14," 2017. [Online]. Available: http://www.3gpp.org/ release- 14

[36] Q. Technologies, "The essential role of Gigabit LTE \& LTE Advanced Pro in a 5G World Our vision for 5G is a unifying connectivity fabric," Tech. Rep. February, 2017.

[37] Qualcomm, "Accelerating 5G NR for Enhanced Mobile Broadband," Tech. Rep. March, 2017.

[38] 3GPP, "Release 15," 2017. [Online]. Available: http://www.3gpp.org/ release- 15

[39] F. Khan, Z. Pi, and S. Rajagopal, "Millimeter-wave mobile broadband with large scale spatial processing for $5 \mathrm{G}$ mobile communication," in Proc. Annu. Allert. Conf. Commun. Control. Comput., IL, USA, 2012, pp. 1517-1523.

[40] H. Mehrpouyan, M. Matthaiou, R. Wang, G. K. Karagiannidis, and Y. Hua, "Hybrid Millimeter-Wave Systems : A Novel Paradigm for HetNets," IEEE Commun. Mag., vol. 53, no. 1, pp. 216-221, 2015.

[41] J. G. Andrews, T. Bai, M. Kulkarni, A. Alkhateeb, A. Gupta, and R. W. Heath, "Modeling and Analyzing Millimeter Wave Cellular Systems," IEEE Trans. Commun., vol. 65, no. 1, pp. 403-430, jan 2017.

[42] O. E. Ayach, S. Rajagopal, S. Abu-Surra, Z. Pi, and R. W. Heath, "Spatially sparse precoding in millimeter wave MIMO systems," IEEE Trans. Wirel. Commun., vol. 13, no. 3, pp. 1499-1513, mar 2014.

[43] A. Alkhateeb, J. Mo, N. Gonzalez-Prelcic, and R. W. Heath, "MIMO precoding and combining solutions for millimeter-wave systems," IEEE Commun. Mag., vol. 52, no. 12, pp. 122-131, dec 2014.

[44] R. O. I. Méndez-rial, C. Rusu, N. González-prelcic, A. Alkhateeb, S. Member, and R. W. Heath, "Hybrid MIMO Architectures for Millimeter Wave Communications : Phase Shifters or Switches ?" IEEE Access, vol. 4, pp. 247-267, jan 2016.

[45] A. Osseiran, F. Boccardi, V. Braun, K. Kusume, P. Marsch, M. Maternia, O. Queseth, M. Schellmann, H. Schotten, H. Taoka, H. Tullberg, M. A. Uusitalo, B. Timus, and M. Fallgren, "Scenarios for 5G mobile and wireless communications: the vision of the METIS project," IEEE Commun. Mag., vol. 52, no. 5, pp. 26-35, may 2014.

[46] A. Rahimian and F. Mehran, "Rf link budget analysis in urban propagation microcell environment for mobile radio communication systems link planning," in Wireless Communications and Signal Processing (WCSP), 2011 International Conference on. IEEE, 2011, pp. 1-5.

[47] T. E. Bogale and L. B. Le, "Massive MIMO and mmWave for 5G Wireless HetNet: Potential Benefits and Challenges," IEEE Veh. Technol. Mag., vol. 11, no. 1, pp. 64-75, mar 2016.

[48] J. Jang, M. Chung, S. C. Hwang, Y.-G. Lim, H.-j. Yoon, T. Oh, B.-W Min, Y. Lee, K. S. Kim, C.-B. Chae, and D. K. Kim, "Smart small cell with hybrid beamforming for $5 \mathrm{G}$ : Theoretical feasibility and prototype results," IEEE Wirel. Commun., vol. 23, no. 6, pp. 124-131, 2016.

[49] T. Bogale, L. Le, A. Haghighat, and L. Vandendorpe, "On the number of RF chains and phase shifters, and scheduling design with hybrid analog-digital beamforming," IEEE Trans. Wirel. Commun., vol. 15, no. 5, pp. 3311-3326, may 2016.

[50] H. Q. Ngo, E. G. Larsson, and T. L. Marzetta, "Energy and spectral efficiency of very large multiuser MIMO systems," IEEE Trans. Commun., vol. 61, no. 4, pp. 1436-1449, apr 2013.

[51] A. Alkhateeb, Y. H. Nam, J. C. Zhang, and R. W. Heath, "Massive MIMO combining with switches," IEEE Wirel. Commun. Lett., vol. 5, no. 3 , pp. 232-235, jun 2016.

[52] R. Zi, X. Ge, J. Thompson, C.-X. Wang, H. Wang, and T. Han, "Energy Efficiency Optimization of 5G Radio Frequency Chain Systems," IEEE J. Sel. Areas Commun., vol. 34, no. 4, pp. 758-771, apr 2016.

[53] S. Payami, M. Ghoraishi, and M. Dianati, "Hybrid Beamforming for Large Antenna Arrays With Phase Shifter Selection," IEEE Trans. Wirel. Commun., vol. 15, no. 11, pp. 7258-7271, 2016. 
[54] F. Sohrabi and W. Yu, "Hybrid beamforming with finite-resolution phase shifters for large-scale MIMO systems," in Proc. IEEE 16th Int. Work. Signal Process. Adv. Wirel. Commun., no. 1, Stockholm, Sweden, 2015, pp. 136-140.

[55] S. Han, C. L. Io, C. Rowell, Z. Xu, S. Wang, and Z. Pan, "Large scale antenna system with hybrid digital and analog beamforming structure," in Proc. IEEE Int. Conf. Commun. Work. ICC 2014, 2014, pp. 842-847.

[56] Y. Niu, Y. Li, D. Jin, L. Su, and A. V. Vasilakos, "A Survey of Millimeter Wave communications (mmWave) for 5G: Opportunities and Challenges," Wirel. Networks, vol. 21, no. 8, pp. 2657-2676, apr 2015.

[57] K. Zheng, L. Zhao, J. Mei, B. Shao, W. Xiang, and L. Hanzo, "Survey of Large-Scale MIMO Systems," IEEE Commun. Surv. Tutorials, vol. 17, no. 3, pp. 1738-1760, 2015.

[58] R. N. Mitra and D. P. Agrawal, "5G mobile technology: A survey," ICT Express, vol. 1, no. 3, pp. 132-137, jan 2015.

[59] W. Roh, J. Y. Seol, J. Park, B. Lee, J. Lee, Y. Kim, J. Cho, K. Cheun, and F. Aryanfar, "Millimeter-wave beamforming as an enabling technology for 5G cellular communications: Theoretical feasibility and prototype results," IEEE Commun. Mag., vol. 52, no. 2, pp. 106-113, feb 2014.

[60] A. Gupta and R. K. Jha, "A Survey of 5G Network: Architecture and Emerging Technologies," IEEE Access, vol. 3, pp. 1206-1232, aug 2015.

[61] N. Panwar, S. Sharma, and A. K. Singh, "A survey on 5G: The next generation of mobile communication," Phys. Commun., vol. 18, pp. 64-84, nov 2016.

[62] H. Kim and S. Lee, "A Survey on the Key Enabling Technologies for the 5G Mobile Broadband Networks," pp. 27-36, 2016. [Online]. Available: citeseerx.ist.psu.edu

[63] M. Agiwal, A. Roy, and N. Saxena, "Next generation 5G wireless networks: A comprehensive survey," IEEE Commun. Surv. Tutorials, vol. 18 , no. 3, pp. 1617-1655, 2016.

[64] P. Pirinen, "A Brief Overview of 5G Research Activities," in Proc. 1st Int. Conf. 5G Ubiquitous Connect., Levi, Finland, 2014, pp. 17-22.

[65] D. C. Araújo, T. Maciel, J. C. Mota, T. Maksymyuk, A. L. de Almeida, and M. Jo, "Massive MIMO: survey and future research topics," IET Commun., vol. 10, no. 15, pp. 1938-1946, oct 2016.

[66] O. Elijah, S. Member, C. Y. Leow, T. A. Rahman, S. Nunoo, and S. Z. Iliya, "A comprehensive survey of pilot contamination in Massive MIMO-5G system," IEEE Commun. Surv. Tutorials, vol. 18, no. 2, pp. 905-923, 2016.

[67] M. H. Alsharif and R. Nordin, "Evolution towards fifth generation (5G) wireless networks: Current trends and challenges in the deployment of millimetre wave, massive MIMO, and small cells," Telecommun. Syst., pp. 1-21, jul 2016.

[68] D. Muirhead, M. Imran, and K. Arshad, "A Survey on the Challenges, Opportunities and Use of Multiple Antennas in Current and Future 5G Small Cell Base Stations," IEEE Access, vol. 4, pp. 2952-2964, jul 2016.

[69] F. Han, S. Zhao, L. Zhang, and J. Wu, "Survey of Strategies for Switching Off Base Stations in Heterogeneous Networks for Greener 5G Systems," IEEE Access, vol. 4, no. c, pp. 4959-4973, aug 2016.

[70] M. Jaber, M. A. Imran, R. Tafazolli, and A. Tukmanov, "5g backhaul challenges and emerging research directions: A survey," IEEE Access, vol. 4, pp. 1743-1766, 2016.

[71] _ _ "5G Backhaul Challenges and Emerging Research Directions: A Survey," IEEE Access, vol. 4, no. c, pp. 1743-1766, may 2016.

[72] M. Noura and R. Nordin, "A survey on interference management for Device-to-Device (D2D) communication and its challenges in 5G networks," J. Netw. Comput. Appl., vol. 71, pp. 130-150, 2016.

[73] S. Buzzi, I. Chih-Lin, T. E. Klein, H. V. Poor, C. Yang, and A. Zappone, "A survey of energy-efficient techniques for $5 \mathrm{G}$ networks and challenges ahead," IEEE J. Sel. Areas Commun., vol. 34, no. 4, pp. 697-709, apr 2016.

[74] D. Jiang and B. Natarajan, "Hybrid precoding with compressive sensing based limited feedback in massive MIMO systems," Trans. Emerg. Telecommun. Technol., vol. 27, no. 12, pp. 1672-1678, aug 2016.

[75] J. Jing, C. Xiaoxue, and X. Yongbin, "Energy-efficiency based downlink multi-user hybrid beamforming for millimeter wave massive MIMO system," J. China Univ. Posts Telecommun., vol. 23, no. 4, pp. 53-62, aug 2016.

[76] F. Sohrabi and W. Yu, "Hybrid digital and analog beamforming design for large-scale MIMO systems," in Proc. IEEE Int. Conf. Acoust. Speech Signal Process., South Brisbane, QLD, 2015, pp. 2929-2933.
[77] H.-L. Chiang, T. Kadur, W. Rave, and G. Fettweis, "Low-complexity spatial channel estimation and hybrid beamforming for millimeter wave links," in Proc. IEEE 27th Annu. Int. Symp. Pers. Indoor, Mob. Radio Commun., Valencia, Spain, sep 2016, pp. 1-7.

[78] H.-1. Chiang, W. Rave, T. Kadur, and G. Fettweis, "Full Rank Spatial Channel Estimation at Millimeter Wave Systems," in Proc. Int. Symp. Wirel. Commun. Syst., Poznan, Poland, 2016, pp. 42-48.

[79] M. N. Kulkarni, S. Member, A. Ghosh, and J. G. Andrews, "A Comparison of MIMO Techniques in Downlink Millimeter Wave Cellular Networks With Hybrid Beamforming," IEEE Trans. Commun., vol. 64, no. 5, pp. 1952-1967, may 2016.

[80] A. Alkhateeb and R. W. H. Jr., "Frequency Selective Hybrid Precoding for Limited Feedback Millimeter Wave Systems," IEEE Trans. Commun., vol. 64, no. 5, pp. 1801-1818, may 2016.

[81] A. Kaushik, J. Thompson, and M. Yaghoobi, "Sparse Hybrid Precoding and Combining in Millimeter Wave MIMO Systems," in Proc. Radio Propag. Technol. 5G, Durham, UK, 2016, pp. 1-7.

[82] J. Palacios, D. D. Donno, D. Giustiniano, and J. Widmer, "Speeding Up mmWave Beam Training through Low-Complexity Hybrid Transceivers," in Proc. IEEE 27th Annu. Int. Symp. Pers. Indoor, Mob. Radio Commun., Valencia, Spain, 2016, pp. 1-7.

[83] A. Alkhateeb, O. El Ayach, G. Leus, and R. W. Heath, "Hybrid precoding for millimeter wave cellular systems with partial channel knowledge," in Proc. Inf. Theory Appl. Work., San Diego, CA, 2013, pp. $1-5$.

[84] F. Sohrabi and W. Yu, "Hybrid digital and analog beamforming design for large-scale antenna arrays," IEEE J. Sel. Top. Signal Process., vol. 10 , no. 3, pp. 501-513, apr 2016.

[85] G. Zhu, K. Huang, V. K. N. Lau, B. Xia, X. Li, and S. Zhang, "Hybrid Interference Cancellation in Millimeter-Wave MIMO Systems," in Proc. IEEE Int. Conf. Commun. Syst., Shenzhen, China, 2016, pp. 1-6.

[86] C.-H. Chen, C.-R. Tsai, Y.-H. Liu, W.-L. Hung, and A.-Y. Wu, "Compressive Sensing (CS)-Assisted Low-Complexity Beamspace Hybrid Precoding for Millimeter-Wave MIMO Systems," IEEE Trans. Signal Process., vol. 65, no. 6, pp. 1-1, 2016.

[87] Y. Ren, Y. Wang, C. Qi, and Y. Liu, "Multiple-Beam Selection with Limited Feedback for Hybrid Beamforming in Massive MIMO Systems," IEEE Access, vol. 3536, no. c, pp. 1-1, 2017.

[88] C.-E. Chen, "An Iterative Hybrid Transceiver Design Algorithm for Millimeter Wave MIMO Systems," IEEE Wirel. Commun. Lett., vol. 4 , no. 3, pp. 285-288, jun 2015.

[89] Z. Xiao, P. Xia, and X.-G. Xia, "Codebook Design for Millimeter-Wave Channel Estimation with Hybrid Precoding Structure," IEEE Trans. Wirel. Commun., vol. 16, no. 1, pp. 141-153, 2017.

[90] M.-C. Lee and W.-H. Chung, "Adaptive Multimode Hybrid Precoding for Single-RF Virtual Space Modulation with Analog Phase Shift Network in MIMO Systems," IEEE Trans. Wirel. Commun., vol. 1276, no. c, pp. 1-1, 2017.

[91] Y. Y. Lee, C. H. Wang, and Y. H. Huang, "A Hybrid RF/baseband precoding processor based on parallel-index-selection matrix-inversionbypass simultaneous orthogonal matching pursuit for millimeter wave MIMO systems," IEEE Trans. Signal Process., vol. 63, no. 2, pp. 305317, 2015.

[92] X. Zhang, A. F. Molisch, and S. Y. Kung, "Variable-phase-shift-based RF-baseband codesign for MIMO antenna selection," IEEE Trans. Signal Process., vol. 53, no. 11, pp. 4091-4103, 2005.

[93] D. Ying, F. W. Vook, T. A. Thomas, and D. J. Love, "Hybrid structure in massive MIMO: Achieving large sum rate with fewer RF chains," in Proc. IEEE Int. Conf. Commun., London, UK, 2015, pp. 2344-2349.

[94] J.-C. Chen, "Hybrid Beamforming with Discrete Phase Shifters for Millimeter???Wave Massive MIMO Systems," IEEE Trans. Veh. Technol., vol. PP, no. 99, pp. 1-5, 2017. [Online]. Available: http://ieeexplore.ieee.org/document/7858800/

[95] J. Singh and S. Ramakrishna, "On the feasibility of beamforming in millimeter wave communication systems with multiple antenna arrays," in 2014 IEEE Glob. Commun. Conf., Austin, TX, 2014, pp. 3802-3808.

[96] C. Kim, T. Kim, and J. Y. Seol, "Multi-beam transmission diversity with hybrid beamforming for MIMO-OFDM systems," in Proc. IEEE Globecom Work. GC Wkshps, Atlanta, GA, 2013, pp. 61-65.

[97] C. Kim, J. S. Son, T. Kim, and J. Y. Seol, "On the hybrid beamforming with shared array antenna for mmWave MIMO-OFDM systems," in Proc. IEEE Wirel. Commun. Netw. Conf. WCNC, Istanbul, Turkey, 2014, pp. 335-340.

[98] Z. Xu, S. Han, and Z. Pan, "Alternating beamforming methods for hybrid analog and digital MIMO transmission," in Proc. IEEE Int. Conf. Commun., London, UK, 2015, pp. 1595-1600. 
[99] Ö. T. Demir and T. E. Tuncer, "Hybrid Beamforming two Bit RF Phase Shifters in Single Group Multicasting," in Proc. IEEE Int. Conf. Acoust. Speech Signal Process., Ankara, Turkey, 2016, pp. 3271-3275.

[100] G. Wang and G. Ascheid, "Hybrid Beamforming under Equal Gain Constraint for Maximizing Sum Rate at $60 \mathrm{GHz}$," in Proc. IEEE 81st Veh. Technol. Conf. (VTC Spring), Glasgow, UK, 2015, pp. 1-5.

[101] S. He, C. Qi, Y. Wu, and Y. Huang, "Energy-Efficient Transceiver Design for Hybrid Sub-Array Architecture MIMO Systems," IEEE Access, vol. 4, pp. 9895-9905, jan 2017.

[102] M. S. Rahman and K. Josiam, "Low complexity RF beam search algorithms for millimeter-wave systems," 2014 IEEE Glob. Commun. Conf., pp. 3815-3820, 2014.

[103] J. A. Zhang, X. Huang, V. Dyadyuk, and Y. J. Guo, "Massive hybrid antenna array for millimeter-wave cellular communications," IEEE Wirel. Commun., vol. 22, no. 1, pp. 79-87, 2015.

[104] L. K. Chiu and S. H. Wu, "Hybrid radio frequency beamforming and baseband precoding for downlink MU-MIMO mmWave channels," IEEE Int. Conf. Commun., pp. 1346-1351, 2015.

[105] R. A. Stirling-Gallacher and M. S. Rahman, "Multi-user MIMO strategies for a millimeter wave communication system using hybrid beam-forming," in Proc. IEEE Int. Conf. Commun., 2015, pp. 24372443.

[106] S. Park, A. Alkhateeb, and R. W. Heath, "Dynamic Subarrays for Hybrid Precoding in Wideband mmWave MIMO Systems," arXiv Prepr. arXiv1606.08405, pp. 1-30, jun 2016. [Online]. Available: http://arxiv.org/abs/1606.08405

[107] X. Yu, J. C. Shen, J. Zhang, and K. B. Letaief, "Alternating Minimization Algorithms for Hybrid Precoding in Millimeter Wave MIMO Systems," IEEE J. Sel. Top. Signal Process., vol. 10, no. 3, pp. 485500 , feb 2016

[108] X. Gao, L. Dai, S. Han, I. Chih-Lin, and R. W. Heath, "Energyefficient hybrid analog and digital precoding for mmwave mimo systems with large antenna arrays," IEEE Journal on Selected Areas in Communications, vol. 34, no. 4, pp. 998-1009, 2016.

[109] F. Sohrabi and W. Yu, "Hybrid Analog and Digital Beamforming for," in Proc. IEEE 17th Int. Work. Signal Process. Adv. Wirel. Commun., no. 978,2016 , pp. 1-5.

[110] X. Yu, J.-c. Shen, J. Zhang, and K. B. Letaief, "Hybrid Precoding Design in Millimeter Wave MIMO Systems : An Alternating Minimization Approach," in Proc. IEEE Glob. Commun. Conf., San Diego, CA, 2015, pp. 1-6.

[111] O. T. Demir and T. E. Tuncer, "Antenna Selection and Hybrid Beamforming for Simultaneous Wireless Information and Power Transfer in Multi-Group Multicasting Systems," IEEE Trans. Wirel. Commun., vol. 15, no. 10, pp. 6948-6962, 2016.

[112] K.-N. Hsu, C.-H. Wang, Y.-Y. Lee, and Y.-H. Huang, "Low complexity hybrid beamforming and precoding for 2D planar antenna array mmWave systems," in 2015 IEEE Work. Signal Process. Syst. IEEE, oct 2015, pp. 1-6. [Online]. Available: http://ieeexplore.ieee.org/document/7345029/

[113] R. Méndez-Rial, C. Rusu, A. Alkhateeb, N. González-Prelcic, and R. W. Heath, "Channel estimation and hybrid combining for mmWave: phase shifters or switches?" in Proc. Inf. Theory Appl. Work. (ITA), 2015, San Diego, CA, 2015, pp. 90-97.

[114] N. Song, T. Yang, and H. S. Member, "Overlapped Sub-Array based Hybrid Beamforming for Millimeter Wave Multi-User Massive MIMO," IEEE Signal Process. Lett., vol. 24, no. 5, pp. 1-5, 2017.

[115] K. Girim, S. Yeonggyu, P. Hyuncheol, and H. M. Kwon, "Design of Millimeter Wave Hybrid Beamforming Systems," in Proc. Veh. Technol. Conf. (VTC Fall), 2014 IEEE 80th, Vancouver, Canada, 2014, pp. 1-5.

[116] W. Ni and X. Dong, "Hybrid block diagonalization for massive multiuser MIMO systems," IEEE Trans. Commun., vol. 64, no. 1, pp. 201-211, jan 2016.

[117] A. Alkhateeb, O. El Ayach, G. Leus, and R. W. Heath, "Channel estimation and hybrid precoding for millimeter wave cellular systems," IEEE J. Sel. Top. Signal Process., vol. 8, no. 5, pp. 831-846, oct 2014.

[118] C. Rusu, R. Mendez-Rial, N. Gonzalez-Prelcic, and R. Heath, "Low Complexity Hybrid Precoding Strategies for Millimeter Wave Communication Systems," IEEE Trans. Wirel. Commun., vol. 15, no. 12, pp. $8380-8393$, dec 2016.

[119] C.-s. Wu, C.-h. Chen, C.-r. Tsai, and A.-y. A. Wu, "Joint RF / Baseband Grouping-based Codebook Design for Hybrid Beamforming in mmWave MIMO Systems," in Proc. IEEE Int. Conf. Signal Process. Commun. Comput., 2016.

[120] J. Song, J. Choi, and D. J. Love, "Common Codebook Millimeter Wave Beam Design: Designing Beams for Both Sounding and Communica- tion with Uniform Planar Arrays," IEEE Trans. Commun., vol. 65 , no. 4, pp. 1869-1872, 2017.

[121] A. Alkhateeb, R. W. Heath, and G. Leus, "Achievable rates of multiuser millimeter wave systems with hybrid precoding," in 2015 IEEE Int. Conf. Commun. Work. IEEE, jun 2015, pp. 1232-1237.

[122] R. Rajashekar and L. Hanzo, "Iterative Matrix Decomposition Aided Block Diagonalization for mm-Wave Multiuser MIMO Systems," IEEE Trans. Wirel. Commun., vol. PP, no. 99, pp. 1-13, 2016.

[123] W. Ni, X. Dong, and W.-s. Lu, "Near-Optimal Hybrid Processing for Massive MIMO Systems via Matrix Decomposition," Arxiv, pp. 1-10, 2015.

[124] T. E. Bogale, L. B. Le, and X. Wang, "Hybrid analog-digital channel estimation and beamforming: Training-throughput tradeoff," IEEE Trans. Commun., vol. 63, no. 12, pp. 5235-5249, dec 2015.

[125] V. Venkateswaran, F. Pivit, and L. Guan, "Hybrid RF and Digital Beamformer for Cellular Networks : Algorithms, Microwave Architectures, and Measurements," IEEE Trans. Microw. Theory Tech., vol. 64, no. 7, pp. 2226-2243, jul 2016.

[126] L.-F. Lin, W.-H. Chung, H.-J. Chen, and T.-S. Lee, "Energy Efficient Hybrid Precoding for Multi-User Massive MIMO Systems Using LowResolution ADCs," in Proc. IEEE Int. Work. Signal Process. Syst., Dallas, TX, 2016, pp. 115-120.

[127] J. Mo, A. Alkhateeb, S. Abu-surra, and R. W. Heath, "Hybrid Architectures with Few-Bit ADC Receivers : Achievable Rates and Energy-Rate Tradeoffs," IEEE Trans. Wirel. Commun., vol. 16, no. 4, pp. 2274-2287, 2016.

[128] J. Singh and S. Ramakrishna, "On the Feasibility of Codebook-Based Beamforming in Millimeter Wave Systems With Multiple Antenna Arrays," IEEE Trans. Wirel. Commun., vol. 14, no. 5, pp. 2670-2683, 2015.

[129] S. Dutta, M. Mezzavilla, R. Ford, M. Zhang, S. Rangan, and M. Zorzi, "Frame Structure Design and Analysis for Millimeter Wave Cellular Systems," IEEE Trans. Wirel. Commun., vol. PP, no. 99, pp. 1-13, 2017. [Online]. Available: http://arxiv.org/abs/1512.05691

[130] T. Xie, L. Dai, X. Gao, M. Z. Shakir, and Z. Wang, "GMDBased Hybrid Precoding For Millimeter-Wave Massive MIMO Systems," ARXIV Prepr., pp. 1-5, 2016. [Online]. Available: http://arxiv.org/abs/1605.04686

[131] L. Dai, X. Gao, J. Quan, S. Han, and I. Chih-Lin, "Near-optimal hybrid analog and digital precoding for downlink mmWave massive MIMO systems," in IEEE Int. Conf. Commun., London, UK, 2015, pp. 13341339.

[132] R. H. Walden, "Analog-to-Digital Converter Survey and Analysis," IEEE J. Sel. Areas Commun., vol. 17, no. 4, pp. 539-550, 1999.

[133] K. Roth, J. A. Nossek, and L. Fellow, "Achievable Rate and Energy Efficiency of Hybrid and Digital Beamforming Receivers with Low Resolution ADC,' ARXIV Prepr., pp. 1-13, 2016.

[134] W. Abbas, F. Gomez-cuba, and M. Z. Fellow, "Millimeter Wave Receiver Efficiency : A Comprehensive Comparison of Beamforming Schemes with Low Resolution ADCs," ARXIV Prepr., pp. 1-30, 2017.

[135] C. Studer, S. Member, G. Durisi, and S. Member, "Quantized Massive MU-MIMO-OFDM Uplink," IEEE Trans. Commun., vol. 64, no. 6, pp. 2387-2399, 2016.

[136] L. Liang, W. Xu, X. Dong, S. Member, and W. Xu, "Low-complexity hybrid precoding in massive multiuser MIMO systems," IEEE Wirel. Commun. Lett., vol. 3, no. 6, pp. 1-5, dec 2014.

[137] S. Suyama, T. Okuyama, Y. Inoue, and Y. Kishiyama, "5G multiantenna technology," NTT DOCOMO Tech. J., vol. 17, no. 4, pp. 29-39, 2016.

[138] E. Yaacoub, M. Husseini, and H. Ghaziri, "An overview of research topics and challenges for 5G massive MIMO antennas," in 2016 IEEE Middle East Conf. Antennas Propag. IEEE, sep 2016, pp. 1-4. [Online]. Available: http://ieeexplore.ieee.org/document/7790121/

[139] U. Johannsen, "Technologies for integrated millimeter-wave antennas," Ph.D. dissertation, Technische Universiteit Eindhoven, 2013.

[140] J. Song, J. Choi, and D. J. Love, "Codebook design for hybrid beamforming in millimeter wave systems," in Proc. IEEE Int. Conf. Commun., London, UK, 2015, pp. 1298-1303.

[141] D. C. Araujo, E. Karipidis, A. L. F. De Almeida, and J. C. M. Mota, "Improving spectral efficiency in large-array FDD systems with hybrid beamforming," in Proc. IEEE Sens. Array Multichannel Signal Process. Work., Rio, Brazil, 2016.

[142] J. Lee, G. T. Gil, and Y. H. Lee, "Channel Estimation via Orthogonal Matching Pursuit for Hybrid MIMO Systems in Millimeter Wave Communications," IEEE Trans. Commun., vol. 64, no. 6, pp. 23702386, jun 2016. 
[143] K. Venugopal, A. Alkhateeb, N. G. Prelcic, and R. W. Heath, "Channel Estimation for Hybrid Architecture Based Wideband Millimeter Wave Systems," Submitt. to IEEE J. Sel. Areas Commun., vol. 0, no. 0, pp. 1-31, 2016. [Online]. Available: http://arxiv.org/abs/1611.03046

[144] R. Rajashekar and L. Hanzo, "Hybrid Beamforming in mm-Wave MIMO Systems Having a Finite Input Alphabet," IEEE Trans. Commun., vol. 64, no. 8, pp. 3337-3349, aug 2016.

[145] S. Noh, M. D. Zoltowski, and D. J. Love, "Training sequence design for feedback assisted hybrid beamforming in massive MIMO systems," IEEE Trans. Commun., vol. 64, no. 1, pp. 187-200, jan 2016.

[146] A. Liu and V. K. N. Lau, "Impact of CSI Knowledge on the CodebookBased Hybrid Beamforming in Massive MIMO," IEEE Trans. Signal Process., vol. 64, no. 24, pp. 6545-6556, dec 2016.

[147] A. Alkhateeb, S. Member, G. Leus, and R. W. Heath, "Limited Feedback Hybrid Precoding for Multi-User Millimeter Wave Systems,", IEEE Trans. Wirel. Commun., vol. 14, no. 11, pp. 6481-6494, nov 2015.

[148] Z. Gao, C. Hu, L. Dai, and Z. Wang, "Channel Estimation for Millimeter-Wave Massive MIMO with Hybrid Precoding over Frequency-Selective Fading Channels," IEEE Commun. Lett., vol. 20 no. 6, pp. 1259-1262, jun 2016.

[149] Z. Gao, L. Dai, D. Mi, Z. Wang, M. A. Imran, and M. Z. Shakir, "MmWave massive-MIMO-based wireless backhaul for the 5G ultradense network," IEEE Wirel. Commun., vol. 22, no. 5, pp. 13-21, oct 2015

[150] M. Borga, "Learning multidimensional signal processing," Ph.D. dissertation, Linkoping University, 1998. [Online]. Available: http: //www.diva-portal.org/smash/record.jsf?pid=diva2:302872

[151] J. Brady, N. Behdad, and A. M. Sayeed, "Beamspace MIMO for Millimeter-Wave Communications: System Architecture, Modeling, Analysis, and Measurements," IEEE Trans. Antennas Propag., vol. 61 , no. 7, pp. 3814-3827, jul 2013.

[152] J. Zhang, A. Wiesel, and M. Haardt, "Low rank approximation based hybrid precoding schemes for multi-carrier single-user massive MIMO systems," in Proc. IEEE Int. Conf. Acoust. Speech Signal Process., Shanghai, China, mar 2016, pp. 3281-3285.

[153] R. Mai, D. H. N. Nguyen, and T. Le-Ngoc, "MMSE hybrid precoder design for millimeter-wave massive MIMO systems," in Proc. IEEE Wirel. Commun. Netw. Conf. WCNC, Doha, Qatar, 2016, pp. 1-7.

[154] E. Zochmann, S. Schwarz, and M. Rupp, "Comparing antenna selection and hybrid precoding for millimeter wave wireless communications," in Proc. IEEE Sens. Array Multichannel Signal Process. Work., Rio, Brazil, jul 2016, pp. 1-5.

[155] A. Roze, M. Crussiere, M. Helard, and C. Langlais, "Comparison between a hybrid digital and analog beamforming system and a fully digital Massive MIMO system with adaptive beamsteering receivers in millimeter-Wave transmissions," in Proc. Int. Symp. Wirel. Commun. Syst., Poznan, Poland, 2016, pp. 86-91.

[156] Z. C. Phyo and A. Taparugssanagorn, "Hybrid analog-digital downlink beamforming for massive MIMO system with uniform and nonuniform linear arrays," in Proc. 13th Int. Conf. Electr. Eng. Comput. Telecommun. Inf. Technol., Chiang Mai, Thiland, jun 2016, pp. 1-6.

[157] G. Wang, J. Sun, and G. Ascheid, "Hybrid Beamforming with Time Delay Compensation for Millimeter Wave MIMO Frequency Selective Channels," in Proc. IEEE 83rd Veh. Technol. Conf. (VTC Spring), Nanjing, China, may 2016, pp. 1-6.

[158] S. Han, I. Chih-Lin, Z. Xu, and S. Wang, "Reference signals design for hybrid analog and digital beamforming," IEEE Commun. Lett., vol. 18, no. 7, pp. 1191-1193, jul 2014

[159] P. V. Amadori and C. Masouros, "Low RF-Complexity MillimeterWave Beamspace-MIMO Systems by Beam Selection," IEEE Trans. Commun., vol. 63, no. 6, pp. 2212-2223, jun 2015

[160] J. Cai, B. Rong, and S. Sun, "A low complexity hybrid precoding scheme for massive MIMO system," in Proc. 16th Int. Symp. Commun. Inf. Technol. IEEE, sep 2016, pp. 638-641.

[161] D. Zhu, B. Li, and P. Liang, "A Novel Hybrid Beamforming Algorithm with Unified Analog Beamforming by Subspace Construction Based on Partial CSI for Massive MIMO-OFDM Systems," IEEE Trans. Commun., vol. 6778, no. c, pp. 1-34, 2016

[162] S. Fujio, C. Kojima, T. Shimura, K. Nishikawa, K. Ozaki, Z. Li, A. Honda, S. Ishikawa, T. Ohshima, H. Ashida, M. Shimizu, and Y. Ohashi, "Robust beamforming method for SDMA with interleaved subarray hybrid beamforming," in Proc. IEEE 27th Annu. Int. Symp. Pers. Indoor, Mob. Radio Commun., Valencia, Spain, sep 2016, pp. $1-5$.

[163] D. H. N. Nguyen, L. B. Le, and T. Le-ngoc, "Hybrid MMSE Precoding for mmWave Multiuser MIMO Systems," in Proc. IEEE Int. Conf. Commun., Kuala Lumpur, Malaysia, 2016, pp. 1-6.
[164] A. Garcia-Rodriguez, V. Venkateswaran, P. Rulikowski, and C. Masouros, "Hybrid analog-digital precoding revisited under realistic RF modeling," IEEE Wirel. Commun. Lett., vol. 5, no. 5, pp. 528-531, oct 2016.

[165] J. Jiang and D. Kong, "Joint User Scheduling and MU-MIMO Hybrid Beamforming Algorithm for mmWave FDMA Massive MIMO System," Int. J. Antennas Propag., vol. 2016, pp. 1-10, oct 2016.

[166] A. Alkhateeb, G. Leus, and R. W. Heath, "Multi-Layer Precoding : A Potential Solution for Full-Dimensional Massive MIMO Systems," Submitt. to IEEE Trans. Wirel. Commun., vol. 0, no. 0, pp. 1-1, 2016.

[167] G. Zhu, K. Huang, V. K. Lau, B. Xia, X. Li, and S. Zhang, "Hybrid Beamforming via the Kronecker Decomposition for the MillimeterWave Massive MIMO Systems," IEEE Journal on Selected Areas in Communications, vol. 35, no. 9, pp. 2097-2114, 2017.

[168] S. Gimenez, S. Roger, D. Mart, J. F. Monserrat, P. Baracca, V. Braun, H. Halbauer, and B. Labs, "Performance of Hybrid Beamforming for mmW Multi-antenna Systems in Dense Urban Scenarios," in Proc. IEEE 27th Annu. Int. Symp. Pers. Indoor, Mob. Radio Commun., Valencia, Spain, sep 2016, pp. 1-6.

[169] A. Kammoun, H. Khanfir, Z. Altman, M. Debbah, and M. Kamoun, "Preliminary Results on 3D Channel Modeling: From Theory to Standardization," IEEE J. Sel. Areas Commun., vol. 32, no. 6, pp. 1219-1229, jun 2014.

[170] J. Li, L. Xiao, X. Xu, and S. Zhou, "Robust and low complexity hybrid beamforming for uplink multiuser mmWave MIMO systems," IEEE Commun. Lett., vol. 20, no. 6, pp. 1140-1143, jun 2016.

[171] W. Feng, Y. Li, D. Jin, L. Su, and S. Chen, "Millimetre-wave backhaul for 5G networks: Challenges and solutions," Sensors (Switzerland), vol. 16, no. 6, pp. 1-17, 2016.

[172] M. Kim and Y. H. Lee, "MSE-Based Hybrid RF/Baseband Processing for Millimeter-Wave Communication Systems in MIMO Interference Channels," IEEE Trans. Veh. Technol., vol. 64, no. 6, pp. 2714-2720, jun 2015.

[173] F. Sohrabi and W. Yu, "Hybrid digital and analog beamforming design for large-scale antenna arrays," IEEE Journal of Selected Topics in Signal Processing, vol. 10, no. 3, pp. 501-513, 2016.

[174] W. Wu, Q. Shen, M. Wang, and X. S. Shen, "Performance analysis of ieee 802.11. ad downlink hybrid beamforming," in Communications (ICC), 2017 IEEE International Conference on. IEEE, 2017, pp. 1-6.

[175] J. A. Tropp, "Algorithms for simultaneous sparse approximation. Part II: Convex relaxation," Signal Processing, vol. 86, no. 3, pp. 589-602, 2006.

[176] A. Adhikary, E. Al Safadi, M. K. Samimi, R. Wang, G. Caire, T. S. Rappaport, and A. F. Molisch, "Joint spatial division and multiplexing for mm-Wave channels," IEEE J. Sel. Areas Commun., vol. 32, no. 6 , pp. 1239-1255, jun 2014

[177] R. Taori and A. Sridharan, "Point-to-Multipoint In-Band mmWave Backhaul for 5G Networks," IEEE Commun. Mag., vol. 53, no. 1, pp. 195-201, 2015.

[178] H. Shokri-Ghadikolaei, C. Fischione, G. Fodor, P. Popovski, and M. Zorzi, "Millimeter wave cellular networks: A MAC layer perspective," IEEE Trans. Commun., vol. 63, no. 10, pp. 3437-3458, 2015.

[179] J. Nam, A. Adhikary, J.-y. Ahn, and G. Caire, "Joint Spatial Division and Multiplexing : Opportunistic Beamforming, User Grouping and Simplified Downlink Scheduling," IEEE J. Sel. Top. Signal Process., vol. 8, no. 5, pp. 876-890, 2014.

[180] S. Sun, G. R. M. Jr, M. K. Samimi, S. Nie, and T. S. Rappaport, "Millimeter Wave Multi-beam Antenna Combining for 5G Cellular Link Improvement in New York City," in Proc. IEEE Int. Conf. Commun., 2014, pp. 5468-5473.

[181] R. Mudumbai, S. Singh, and U. Madhow, "Medium access control for $60 \mathrm{ghz}$ outdoor mesh networks with highly directional links," in INFOCOM 2009, IEEE. IEEE, 2009, pp. 2871-2875.

[182] ECMA International, "High rate $60 \mathrm{GHz}$ PHY, MAC and HDMI PAL," 2008.

[183] IEEE 802.15 WPAN Millimeter Wave Alternative PHY Task Group 3c (TG3c), Available at http://www.ieee802.org/15/pub/TG3c.html.

[184] Draft Standard for Information Technology-Telecommunications and Information Exchange Between Systems-Local and Metropolitan Area Networks-Specific Requirements-Part 11: Wireless LAN Medium Access Control (MAC) and Physical Layer (PHY) SpecificationsAmendment 4: Enhancements for Very High Throughput in the 60 GHz Band, IEEE P802.11ad/ D9.0, Oct 2012.

[185] M. X. Gong, R. Stacey, D. Akhmetov, and S. Mao, "A directiona CSMA/CA protocol for mmwave wireless pans," in Wireless Communications and Networking Conference (WCNC), 2010 IEEE. IEEE, 2010, pp. 1-6. 
[186] H. Park, S. Park, T. Song, and S. Pack, "An incremental multicast grouping scheme for mmwave networks with directional antennas," IEEE Communications Letters, vol. 17, no. 3, pp. 616-619, 2013.

[187] S. Singh, R. Mudumbai, and U. Madhow, "Distributed coordination with deaf neighbors: efficient medium access for $60 \mathrm{ghz}$ mesh networks," in INFOCOM, 2010 Proceedings IEEE. IEEE, 2010, pp. $1-9$.

[188] F. Dai and J. Wu, "Efficient broadcasting in ad hoc wireless networks using directional antennas," IEEE Transactions on Parallel and Distributed Systems, vol. 17, no. 4, pp. 335-347, 2006.

[189] J. Kim, "Elements of next-generation wireless video systems: Millimeter-wave and device-to-device algorithms," Ph.D. dissertation, University of Southern California, 2014.

[190] J. Kim and A. F. Molisch, "Fast millimeter-wave beam training with receive beamforming," Journal of Communications and Networks, vol. 16 , no. 5, pp. 512-522, 2014

[191] X. Hou, X. Wang, H. Jiang, and H. Kayama, "Investigation of massive MIMO in dense small cell deployment for 5G," in Proc. IEEE 84rd Veh. Technol. Conf. (VTC Fall), Montréal, Canada, 2016, pp. 1-6.

[192] B. Xu, Y. Chen, M. Elkashlan, T. Zhang, and K. K. Wong, "User association in massive MIMO and mmWave enabled HetNets powered by renewable energy," in Proc. IEEE Wirel. Commun. Netw. Conf. WCNC, Doha, Qatar, 2016, pp. 1-6.

[193] S.-h. Wu, J.-w. Wang, and J.-y. Chen, "Reconfigurable Hybrid Beamforming for Dual-Polarized mmWave MIMO Channels," in Proc. IEEE 27th Annu. Int. Symp. Pers. Indoor, Mob. Radio Commun., Valencia, Spain, 2016, pp. 1-6.

[194] Z. Pi, J. Choi, and R. Heath, "Millimeter-wave gigabit broadband evolution toward 5G: Fixed access and backhaul," IEEE Commun. Mag., vol. 54, no. 4, pp. 138-144, apr 2016.

[195] S. Hur, T. Kim, D. J. Love, J. V. Krogmeier, T. a. Thomas, and A. Ghosh, "Millimeter wave beamforming for wireless backhaul and access in small cell networks," IEEE Trans. Commun., vol. 61, no. 10, pp. 4391-4403, oct 2013.

[196] W. Liu, S. Han, C. Yang, and C. Sun, "Massive mimo or small cell network: Who is more energy efficient?" in Wireless Communications and Networking Conference Workshops (WCNCW), 2013 IEEE. IEEE, 2013, pp. 24-29.

[197] K. Hosseini, J. Hoydis, S. Ten Brink, and M. Debbah, "Massive mimo and small cells: How to densify heterogeneous networks," in Communications (ICC), 2013 IEEE International Conference on. IEEE, 2013, pp. 5442-5447.

[198] V. Jungnickel, K. Manolakis, W. Zirwas, B. Panzner, V. Braun, M. Lossow, M. Sternad, R. Apelfrojd, and T. Svensson, "The role of small cells, coordinated multipoint, and massive mimo in $5 \mathrm{~g}$," IEEE Communications Magazine, vol. 52, no. 5, pp. 44-51, 2014.

[199] T. E. Bogale and L. B. Le, "Beamforming for Multiuser Massive MIMO Systems : Digital versus Hybrid Analog-Digital," in Proc. IEEE Glob. Commun. Conf., Austin, TX, 2014, pp. 4066-4071.

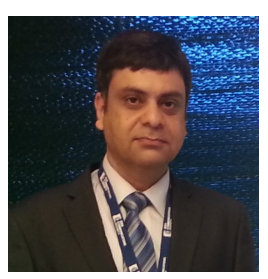

Irfan Ahmed (M'10, SM'16) received the B.E. Electrical Engineering degree and the M.S. Computer Engineering degree from University of Engineering and Technology, Taxila, Pakistan, in 1999 and 2003, respectively, and the $\mathrm{PhD}$ degree in Telecommunication Engineering from the Beijing University of Posts and Telecommunications, Beijing, China, in 2008. Currently, he is working as assistant professor in Higher Colleges of Technology, UAE. He has been working assistant/associate professor in Taif University during 2011-2017. He was post-doctoral fellow with Qatar University from April 2010 to March 2011, where he worked on two research projects, wireless mesh networks with Purdue University, USA, and radio resource allocation for LTE with Qtel. $\mathrm{He}$ has also been involved in National ICT Pakistan funded research project 'Design and development of MIMO and Cooperative MIMO test-bed' at Iqra University, Islamabad, Pakistan, during 2008 to 2010. His research interests include wireless LAN (WLAN) medium access control (MAC) protocol design and analysis, cooperative communications, MIMO communications, performance analysis of wireless channels, energy constrained wireless networks, cognitive radio networks, and radio resource allocation. He is an author of more than 25 International publications. Dr. Irfan served as session chair of the IEEE Wireless Communications, Networking and Mobile Computing conference held in Shanghai, China, in September 2007, IEEE ICC 2016. He is an active reviewer of IEEE, Springer, and Elsevier journals, and conferences. $\mathrm{He}$ is an associate editor of IEEE Access journal.

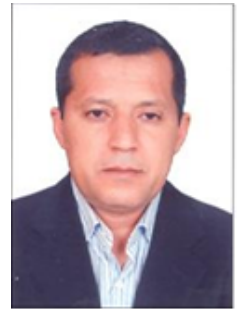

Hedi Khammari received the Bachelor Electrical Engineering degree and the M.S. Automatic Control degree from National College of Engineering of Tunis, Tunisia in 1988 and 1990, respectively, and the $\mathrm{PhD}$ degree in electrical engineering from Tunis University in 1999. He worked as Electrical Engineer in Tunisian company of electricity and gas, from 1989 - 1991. In fall 1992, he worked as a full time lecturer at the faculty of Industry and Mines in Gafsa, Tunisia. In fall 1996, he has been with the electrical engineering in Higher College of technology of Nabeul, Tunisia. In fall 2005, he joined the electrical engineering department of National Engineering College of Monastir, Tunisia as an assistant professor. In spring 2007, he has been in the Higher Institute of Applied Science and Technology of Kairouan, Tunisia. He is currently working as an associate professor in the department of computer engineering, college of computers and information technology, Taif University, Saudi Arabia. His main area of research interests includes nonlinear dynamics, bioinformatics and wireless communication. $\mathrm{He}$ is an active reviewer of Nonlinear Dynamics Springer journal, and conferences. 


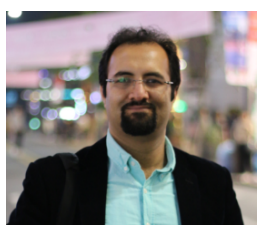

Adnan Shahid (M'15, SM'17) received the B.Eng. and the M.Eng. degrees in computer engineering with wireless communication specialization from the University of Engineering and Technology, Taxila, Pakistan in 2006 and 2010, respectively, and the $\mathrm{Ph} . \mathrm{D}$ degree in information and communication engineering from the Sejong University, South Korea in 2015 . He is currently working as a Postdoctoral Researcher/Senior Researcher at IDLab, a core research group of imec with research activities combined with Ghent University and University of Antwerp. He is involved in several European research projects including eWINE, WiSHFUL, etc and national projects SAMURAI, IDEAL-IOT, etc. $\mathrm{He}$ is also technically coordinating the eWINE project and also involved in future research proposals. From Sep 2015 âĂŞ Jun 2016, he was with the Department of Computer Engineering, Taif University, Saudi Arabia. From Mar 2015 âĂŞ Aug 2015, he worked as a Postdoc Researcher at Yonsei University, South Korea. From Aug 2012 âĂŞ Feb 2015, he worked as a $\mathrm{PhD}$ research assistant in Sejong University, South Korea. From Mar 2007 âĂS Aug 2012, he served as a Lecturer in electrical engineering department of National University of Computer and Emerging Sciences (NUCES-FAST), Pakistan. He was also a recipient of the prestigious BK 21 plus Postdoc and Research Professor at Yonsei University, South Korea. He is a senior member of IEEE and actively involved in various research activities. He is also serving as an Associate Editor at IEEE Access Journal and JNCA, Elsevier. He is an author and co-author of more than $35+$ publications in well-known conferences and journals.

His research interests includes resource management, interference management, cross-layer optimization, self-organizing networks, small cell networks, device to device communications, machine to machine communications, sub$\mathrm{GHz}$ technologies, $5 \mathrm{G}$ wireless communications, machine learning, etc.

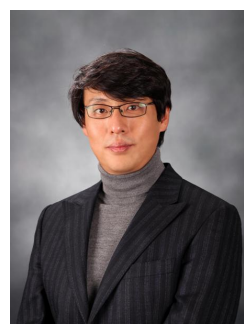

Kwang Soon Kim (S'95, M'99, SM'04) was born in Seoul, Korea, on September 20, 1972. He received the B.S. (summa cum laude), M.S.E., and $\mathrm{Ph} . \mathrm{D}$. degrees in Electrical Engineering from Korea Advanced Institute of Science and Technology (KAIST), Daejeon, Korea, in February 1994, February 1996, and February 1999, respectively. From March 1999 to March 2000, he was with the Department of Electrical and Computer Engineering, University of California at San Diego, La Jolla, CA, U.S.A., as a Postdoctoral Researcher. From April 2000 to February 2004, he was with the Mobile Telecommunication Research Laboratory, Electronics and Telecommunication Research Institute, Daejeon, Korea as a Senior Member of Research Staff. Since March 2004, he has been with the Department of Electrical and Electronic Engineering, Yonsei University, Seoul, Korea, now is a Professor. Prof. Kim is a Senior Member of the IEEE, served as an Editor of the Journal of the Korean Institute of Communications and Information Sciences (KICS) from 2006-2012, as the Editor-in-Chief of the journal of KICS since 2013, as an Editor of the Journal of Communications and Networks (JCN) since 2008, as an Editor of the IEEE Transactions on Wireless Communications 2009-2014. He was a recipient of the Postdoctoral Fellowship from Korea Science and Engineering Foundation (KOSEF) in 1999. He received the Outstanding Researcher Award from Electronics and Telecommunication Research Institute (ETRI) in 2002, the Jack Neubauer Memorial Award (Best system paper award, IEEE Transactions on Vehicular Technology) from IEEE Vehicular Technology Society in 2008, and LG R\&D Award: Industry-Academic Cooperation Prize, LG Electronics, 2013. His research interests are generally in signal processing, communication theory, information theory, and stochastic geometry applied to wireless heterogeneous cellular networks, wireless local area networks, wireless D2D networks and wireless ad doc networks, and are focused on the new radio access technologies for $5 \mathrm{G}$, recently.

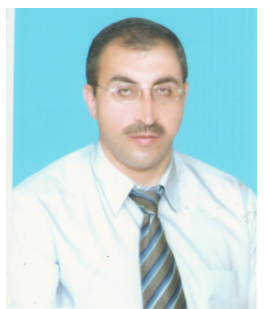

Ahmed Musa received the B.S. and M.S. in 1997 and 2000, respectively, both in Electrical and Computer Engineering from Jordan University of Science and Technology. He worked as Telecom Engineer in OrangeJo from 1999 - 2001. In fall 2001, he joined the Ph.D. program in Computer Engineering at the University of Texas at El Paso (UTEP), USA. In spring 2006, Dr. Musa worked as a full time assistant professor at the faculty of Computer Engineering at Al-Hussein Bin Talal University (AHU), Ma'an, Jordan. In fall 2006, he is charged with leading the Department of Computer Engineering at AHU to the next level of distinction in research and teaching. In fall 2009, he was appointed as a vice dean, faculty of Engineering. During his period as a vice dean, he has participated in many committees on both university and national wise basis. Prior to joining Yarmouk University, he was working as a visiting professor at the department of Computer Engineering, Taif University (TU), Kingdom of Saudi Arabia. Dr. Musa is currently an associate professor of Telecommunications Engineering at Yarmouk University, Irbid, Jordan. Dr. Musa has been served as a reviewer for many Journals and international conferences. His main area of research interests includes optical fiber communications, communications networks (wireless, cognitive radio, etc.) and security, digital signal processing and data compression.

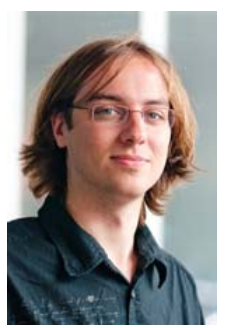

Eli De Poorter is a Professor at Ghent University. He received his master degree in Computer Science Engineering from Ghent University, Belgium, in 2006, his Ph.D. degree in 2011 at the Department of Information Technology at Ghent University and became a full-time professor at Internet and Data Lab research group (IDLab, www.IDLab.ugent.be) in 2015. In addition to teaching several courses, he is involved in and/or research coordinator of multiple national and international projects related to future wireless networks. His main research interests include wireless network protocols, network architectures, wireless sensor an ad hoc networks, future internet, self-learning networks and next-generation network architectures. He is part of the program committee of several conferences and is the author or co-author of more than 90 papers published in international journals or in the proceedings of international conferences. He is also the creator of the patented IDRA architecture, a flexible communication framework for heterogeneous networked devices. 


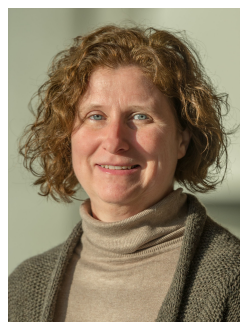

Ingrid Moerman received her degree in Electrical Engineering (1987) and the Ph.D degree (1992) from the Ghent University, where she became a part-time professor in 2000. She is a staff member at IDLab, a core research group of imec with research activities embedded in Ghent University and University of Antwerp. Ingrid Moerman is coordinating the research activities on mobile and wireless networking, and she is leading a research team of about $30 \mathrm{mem}$ bers at IDLab-Ghent University. Her main research interests include: Internet of Things, Low Power Wide Area Networks (LPWAN), High-density wireless access networks, collaborative and cooperative networks, intelligent cognitive radio networks, real-time software defined radio, flexible hardware/software architectures for radio/network control and management, and experimentally-supported research. Ingrid Moerman has a longstanding experience in running and coordinating national and EU research funded projects. At the European level, Ingrid Moerman is in particular very active in the Future Networks research area, where she is coordinating several H2020 projects (WiSHFUL, eWINE, ORCA). Ingrid Moerman is also leading the SCATTER Team that participates in the DARPA Spectrum Collaboration Challenge and was one of the prize winners of the first phase. Ingrid Moerman is author or co-author of more than 700 publications in international journals or conference proceedings. 